\title{
Quantifying the suppression of the (un)-obscured star formation in galaxy cluster cores at $0.2 \lesssim z \lesssim 0.9$
}

\author{
L. Rodríguez-Muñoz, ${ }^{1 \star}$ G. Rodighiero, ${ }^{1}$ C. Mancini, ${ }^{1}$ P. G. Pérez-González, ${ }^{2,3}$ \\ T. D. Rawle, ${ }^{4}$ E. Egami, ${ }^{5}$ A. Mercurio,${ }^{6}$ P. Rosati, ${ }^{7}$ A. Puglisi ${ }^{\odot}, 1,8$ A. Franceschini, ${ }^{1}$ \\ I. Balestra, ${ }^{9}$ I. Baronchelli, ${ }^{1,10}$ A. Biviano, ${ }^{11}$ H. Ebeling, ${ }^{12}$ A. C. Edge, ${ }^{13}$ A. F. M. Enia, ${ }^{1}$ \\ C. Grillo, ${ }^{14,15}$ C. P. Haines ${ }^{\oplus},{ }^{16}$ E. Iani,${ }^{1}$ T. Jones ${ }^{\odot},{ }^{17,18}$ M. Nonino, ${ }^{11}$ I. Valtchanov ${ }^{\odot},{ }^{19}$ \\ B. Vulcani ${ }^{\oplus 20}$ and M. Zemcov ${ }^{21}$
}

Affiliations are listed at the end of the paper

Accepted 2018 December 5. Received 2018 October 31; in original form 2018 February 8

\begin{abstract}
We quantify the star formation (SF) in the inner cores $\left(\mathcal{R} / R_{200} \leq 0.3\right)$ of 24 massive galaxy clusters at $0.2 \lesssim z \lesssim 0.9$ observed by the Herschel Lensing Survey and the Cluster Lensing and Supernova survey with Hubble. These programmes, covering the rest-frame ultraviolet to far-infrared regimes, allow us to accurately characterize stellar mass-limited $\left(\mathcal{M}_{*}>10^{10} M_{\odot}\right)$ samples of star-forming cluster members (not)-detected in the mid- and/or far-infrared. We release the catalogues with the photometry, photometric redshifts, and physical properties of these samples. We also quantify the SF displayed by comparable field samples from the Cosmic Assembly Near-infrared Deep Extragalactic Legacy Survey. We find that in intermediate$z$ cluster cores, the SF activity is suppressed with respect the field in terms of both the fraction $(\mathcal{F})$ of star-forming galaxies (SFGs) and the rate at which they form stars $(\mathcal{S F R}$ and $\left.s \mathcal{S F R}=\mathcal{S F R} / \mathcal{M}_{*}\right)$. On average, the $\mathcal{F}$ of SFGs is a factor $\sim 2$ smaller in cluster cores than in the field. Furthermore, SFGs present average $\mathcal{S F R}$ and $s \mathcal{S F R}$ typically $\sim 0.3$ dex smaller in the clusters than in the field along the whole redshift range probed. Our results favour long time-scale quenching physical processes as the main driver of SF suppression in the inner cores of clusters since $z \sim 0.9$, with shorter time-scale processes being very likely responsible for a fraction of the missing SFG population.
\end{abstract}

Key words: galaxies: clusters: general-galaxies: evolution-galaxies: star formationcatalogues.

\section{INTRODUCTION}

Galaxies appear to be distributed into two fairly distinct general groups (e.g. Kauffmann et al. 2003; Bell et al. 2004; Baldry et al. 2004; Haines et al. 2017): a population of relatively red, quiescent galaxies [i.e. where the star formation (SF) activity has already been quenched], which are characterized by spheroid-dominated morphologies; and a population of rather blue, star-forming galaxies (SFGs), with disc-dominated morphologies. Understanding the nature of the processes that makes a galaxy a member of either category at any cosmological epoch is one of the longest standing unsolved problems in astrophysics.

The fraction of red/quiescent/early-type galaxies among the whole population scales with the stellar mass $\left(\mathcal{M}_{*}\right)$ of the galaxies

^E-mail: lucia.rodriguezmunoz@unipd.it up to $z \sim 4$ (e.g. Baldry et al. 2004, 2006), and with the density of the environments they inhabit at least up to $z \sim 1$ (e.g. Dressler 1980; Lewis et al. 2002). Hence, different works have claimed that this dichotomy between (still) SFGs and quenched galaxies, should be driven (independently, Peng et al. 2010) by the impact on the evolution of galaxies of two kind of processes: those somehow related to the stellar mass of the galaxies they quench, and therefore, responsible for the so-called mass quenching; and those linked to physical processes taking place in high-density environments, responsible for the so-called environmental quenching. The physical nature of these quenching processes and its evolution with redshift remain controversial.

A plethora of works have studied the SF activity within galaxy clusters at different redshifts as to quantify the environmental influence on galaxy evolution (e.g. Dressler et al. 1997; Poggianti et al. 1999; Poggianti 2003; De Lucia et al. 2007; Saintonge, Tran \& 
Holden 2008; Finn et al. 2010; Vulcani et al. 2011). This large body of work gives evidence for a significant transformation of galaxy populations in clusters since $z \sim 1$. Already three decades ago, Butcher \& Oemler (1984, see also Butcher \& Oemler 1978) found that the fraction of blue cluster members increases from zero in the Local Universe to $\sim 20$ percent by $z \sim 0.4$. This rapid evolution over the last 5 billion years can only be explained by the existence of a population of field SFGs entering the cluster environment, which eventually is capable of turning them into passively evolving systems. This scenario is also favoured by the standard hierarchical cosmological model, which predicts a peak in the rate of field galaxies entering the cluster environment at $z \sim 0.4$ (Kauffmann 1995).

In clusters, SFGs are not only less numerous than in the field, but they seem to present also different properties with respect their isolated counterparts. For instance, rich environments host a high fraction of post-starburst (PSB, e.g. Poggianti et al. 2009; Muzzin et al. 2014; Paccagnella et al. 2017), and jellyfish galaxies (e.g. Smith et al. 2010; Poggianti et al. 2017). Also, CO observations in $z \sim 0.4-0.5$ by Jablonka et al. (2013) show that cluster members contain less molecular gas than field galaxies at the same redshift.

Works such as Patel et al. (2009), Vulcani et al. (2010), Haines et al. (2013), or Paccagnella et al. (2016) find a different distribution of star formation rate $(\mathcal{S F R})$, and specific star formation rate ( $s \mathcal{S F R}$; defined as the ratio between the $\mathcal{S F R}$ and the $\mathcal{M}_{*}$ of a galaxy) in the inner regions of clusters (i.e. within the virial radius, $\mathcal{R}_{\text {virial }}$ ) with respect to the field, with values typically $\sim 0.2-0.3 \mathrm{dex}$ smaller for the former. This offset translates into a shift in the tight relation between the $\mathcal{S F R}$ and $\mathcal{M}_{*}$ found for the star-forming field galaxies up to $z \sim 4$ (e.g. Noeske et al. 2007; Rodighiero et al. 2011; Whitaker et al. 2012b; Schreiber et al. 2017). Such a correlation is commonly known as the main sequence (MS) of SFGs. The existence of the MS is interpreted as the proof for a typical mode in which the galaxies form stars (e.g. Renzini \& Peng 2015). The tightness of the correlation ( 0.3 dex scatter; e.g. Whitaker et al. 2012b) is interpreted as a possible consequence of the short time-scale of the dominant quenching process (Peng et al. 2010) moving the field SFGs out of the MS. As a consequence, the displacement of the cluster members MS towards lower $\mathcal{S F R}$ values could imply that the dominant quenching mechanisms in rich environments are different (e.g. slow quenching mechanisms could populate the region below the MS with transition galaxies on their way to be turned off, Haines et al. 2013, 2015; Paccagnella et al. 2016). However, other works such as Peng et al. (2010), Finn et al. (2010), Wijesinghe et al. (2012), or Tyler, Rieke \& Bai (2013) find the same $\mathcal{S F R}$ distribution in clusters as in the field at intermediate redshifts. These discrepancies appear to be due to a combination of different factors such as observational biases (e.g. $\mathcal{S F R}$ detection limit), different sample selection functions, and cluster-to-cluster differences (e.g. Geach et al. 2006; Alberts et al. 2016).

A variety of mechanisms have been proposed as the responsible for environmental quenching (see reviews by, e.g. Boselli \& Gavazzi 2006; Haines et al. 2007): gravitational interactions with the potential well of nearby galaxies or the cluster itself, also known as harassment (Moore et al. 1996); removal and thermal heating of the interstellar medium (ISM) of the galaxies by the interaction with the intra-cluster medium (ICM), the so-called ram-pressure stripping (RPS; Gunn \& Gott 1972; Poggianti et al. 2017); the removal of the hot gas reservoirs of the halo of galaxies, or strangulation, and subsequent halt of the supply of material needed to sustain the SF, leading up to the eventual starvation (Larson, Tinsley \& Caldwell
1980). These mechanisms shape the evolution of galaxies in different time-scales, probably with different efficiency depending on the properties of both galaxies and clusters, and the particular circumstances under which the infall takes place (see e.g. Boselli \& Gavazzi 2006; Berrier et al. 2009). Furthermore, it has also been proposed that the environmental impact on these SFGs starts in early stages of the infall if the accreted galaxies are bound up in small groups (pre-processing; e.g. Haines et al. 2015). Distinguishing among these mechanisms remains challenging, and relies on the detailed study and accurate quantification of the changes suffered by the SF processes and structural properties of the galaxies in rich environments.

Recently, a number of state-of-the-art surveys have targeted massive galaxy clusters at intermediate redshift with the main goal of exploring low-luminosity galaxies at high redshift taking advantage of the gravitational lensing phenomenon (e.g. Hubble Frontier Fields, HFF, Lotz et al. 2017). In this work, we aim at shedding light on the impact of environment on the star-forming activity in galaxies populating clusters by using these surveys to study the cluster inhabitants themselves.

We focus our analysis on $24 \mathrm{X}$-ray-selected (i.e. with total masses $\sim 5$ to $\sim 30 \times 10^{14} M_{\odot}$ ) clusters targeted by the Herschel Lensing Survey (HLS; Egami et al. 2010), a far-infrared (FIR) and submillimetre survey using the ESA Herschel Space Observatory, and the Cluster Lensing and Supernova survey with Hubble (CLASH; Postman et al. 2012), a deep optical and near-infrared (NIR) Hubble Space Telescope (HST) program, as well as by other NIR and mid-infrared (MIR) Spitzer programs. The sample extends between $0.187 \leq z \leq 0.890$, thus, covering a particularly interesting cosmic epoch for the study of environmental quenching.

The wealth and quality of this optical-to-NIR photometric data set allows us to identify cluster galaxies applying a methodology based on photometric redshifts to complement the spectroscopic membership assignment. Furthermore, combining the whole multiwavelength data, we can accurately quantify the average (un)obscured SF hosted by $\mathcal{M}_{*}$-selected samples of cluster SFGs. The use of Herschel observations complementing optical and NIR data guarantees a proper quantification of the SF shrouded by dust.

Indeed, SFGs detected in the MIR and/or FIR often have optical colours consistent with those of passively evolving galaxies and therefore, they are easily missed by studies limited to the optical or NIR regimes. Not quantifying the contribution of these obscured processes can lead to an under estimation of the true level of SF by a factor $\sim 10$ (Duc et al. 2002). This can extremely affect high-density environments studies where, despite the overall reduced SF activity observed, a population of dusty star-forming cluster galaxies has been detected at a wide range of redshifts (e.g. Fadda et al. 2000; Duc et al. 2002; Geach et al. 2006; Marcillac et al. 2007; Saintonge et al. 2008; Bai et al. 2009; Dressler et al. 2009; Haines et al. 2009; Rawle et al. 2010; Biviano et al. 2011; Popesso et al. 2011; Kocevski et al. 2011; Coppin et al. 2011; Rawle et al. 2012b; Alberts et al. 2014, 2016)

Ultimately, we systematically quantify the suppression of the formation activity in galaxy cluster cores with respect the field. For this end, we consistently build reference field samples across the same redshift range by applying the same analysis to the optical-to-FIR publicly available photometry on three of the fields targeted by the Cosmic Assembly Near-infrared Deep Extragalactic Legacy Survey (CANDELS, Grogin et al. 2011; Koekemoer et al. 2011).

This article is organized as follows: Section 2 describes the cluster sample and corresponding data. Section 3 describes our 
approach to combining the different photometric data and building the multiwavelength catalogue which we use to derive photometric redshifts (Section 4) and physical properties of galaxies through an SED-fitting approach (Section 5). In Section 6, we detail our procedure to select cluster members using spectroscopic and photometric redshifts estimations. The final cluster members samples of SFGs are presented in Section 7 and further characterized in Section 8. The quantification of the SF activity in the core of these clusters is discussed in Section 9. Finally, an interpretation of our results is given in Section 10, and a summary and the main conclusions of this work are given in Section 11.

Throughout this work, we assume a flat $\Lambda$ cold dark matter cosmology with $H_{0}=70 \mathrm{kms}^{-1} \mathrm{Mpc}^{-1}, \Omega_{\mathrm{m}}=0.3$, and $\Omega_{\Lambda}=0.7$. Star formation rates and stellar masses are based on a Salpeter (1955) initial mass function (IMF).

The catalogues of star-forming cluster members associated to this paper, including multiwavelength photometry, photometric redshifts, and physical properties, can be downloaded from the public flavour of the RAINBOw Cosmological Database ${ }^{1}$ (PérezGonzález et al. 2008, Barro et al. 2011a,b).

\section{GALAXY CLUSTERS SAMPLE AND DATA}

The HLS (Egami et al. 2010) is a large imaging survey of galaxy clusters in the FIR and submillimetre using the ESA Herschel Space Observatory (Pilbratt et al. 2010). HLS provides deep imaging (see Section 2.3) with the Photodetector Array Camera and Spectrometer (PACS, Poglitsch et al. 2010) and the Spectral and Photometric Imaging REceiver (SPIRE, Griffin et al. 2010 ) for a sample of 65 $\mathrm{X}$-ray-luminous (i.e. massive) clusters of galaxies in the redshift range between $0.2 \lesssim z \lesssim 0.9$. The primary aim of HLS is to observe the most effective gravitational lenses available, probing beyond the confusion limit of the Herschel instruments to observe intrinsically faint, high-redshift sources (e.g. Rex et al. 2010; Rawle et al. 2010). However, the HLS is also a remarkable survey for the study of SF processes taking place within high-density environments (e.g. Rawle et al. 2016, 2014). On the one hand, it targets a significant number of clusters, which avoids deriving misleading results due to cluster-to-cluster variations (e.g. Alberts et al. 2016). On the other hand, the clusters targeted by the HLS span over a redshift range in which these systems are thought to undergo a major evolution due to the transformation of infalling star-forming field galaxies into passive objects (e.g. Kauffmann 1995, Haines et al. 2015).

Among the fields targeted by the HLS, we focus our work on a subsample of 24 clusters (see Table 1) also observed by the CLASH (Postman et al. 2012). CLASH is a Multi-Cycle Treasury Program with the aim of providing ultradeep photometry of 25 X-ray-selected, massive $\left(\sim 5\right.$ to $\left.\sim 30 \times 10^{14} M_{\odot}\right)$ galaxy clusters in a total of 16 passbands using HST ACS/WFC, WFC3/UVIS, and WFC3/IR (see Section 2.1 for details). CLASH clusters are drawn heavily from the Abell and MACS cluster catalogues (Abell 1958; Abell, Corwin \& Olowin 1989; Ebeling, Edge \& Henry 2001; Ebeling et al. 2007, 2010; Mann \& Ebeling 2012).

The wealth of photometric and spectroscopic data available for this galaxy clusters sample, that we call CLASH+HLS, enables the accurate identification and characterization of their galaxy population (e.g. Annunziatella et al. 2016; Maier et al. 2016; Balestra et al. 2016). Indeed, CLASH+HLS clusters have been

${ }^{1}$ http://rainbowx.fis.ucm.es extensively studied in previous works. CLASH photometry together with spectroscopy from different surveys (see Section 2.4) have provided strong constraints on the cluster inner mass distributions and profiles (e.g. Biviano et al. 2013; Annunziatella et al. 2014; Zitrin et al. 2015). Also, their dynamical state and substructures have been analysed through different techniques, such as the SunyaevZel'dovich effect (Sunyaev \& Zel'dovich 1972; Rumsey et al. 2016) and X-ray surface brightness analysis (see Rumsey et al. 2016, and references therein), as well as lensing (e.g. Zitrin et al. 2013; Grillo et al. 2015) and kinematics of galaxy populations (e.g. Girardi et al. 2015). Despite the X-ray selection, that generally favours highly relaxed clusters, the sample is found to be not homogeneously dynamically relaxed (Postman et al. 2012; Rumsey et al. 2016). Finally, a number of works have studied in detail the brightest cluster galaxies (BCGs) of the CLASH+HLS systems. For instance, Donahue et al. $(2015,2016)$ carried out a study on the morphology and SF activity of these peculiar galaxies, using the rest-frame ultraviolet (UV) imaging provided by CLASH (see also Fogarty et al. 2015, 2017). Furthermore, they also characterized the intracluster gas in the vicinity of the BCGs and beyond, by analysing the X-ray emission of the inner cluster cores. Complementary, Rawle et al. (2012a) studied the obscured SF activity undergone by the BCGs of the massive clusters observed by HLS, and its dependence with the X-ray gas cooling times for cool-core (CC) clusters. ${ }^{2}$

In the following subsections, we describe the photometric and spectroscopic data sets available on the cluster fields (see Tables 2 and 3 for a summary of their main characteristics), as well as other ancillary data found in the literature.

\subsection{Hubble optical and NIR photometry}

In this work, we use the $\mathrm{CLASH}^{3}$ photometric data set published by Postman et al. (2012). This data release contains the photometry performed on the HST ACS/WFC (F435W, F475W, F606W, F625W, F775W, F814W, and F850LP), WFC3/UVIS (F225W, F275W, F336W, and F390W), and WFC3/IR (F105W, F110W, F125W, F140W, and F160W) deep imaging of 25 massive intermediateredshift clusters. Object detection and photometry is accomplished using SEXTRACTOR (Bertin \& Arnouts 1996) in dual-image mode using a weighted sum of the ACS/WFC and WFC3/IR images (see Postman et al. 2012 for details on the HST data reduction, catalogue build-up, and main characteristics). These catalogues cover an area of $\sim 5 \mathrm{arcmin}^{2}$, limited by the WFC3/IR images $\left(\sim 2.0 \times 2.3 \mathrm{arcmin}^{2}\right)$, and therefore, they mainly sample the very inner cluster cores. An angular distance of 2.0 arcmin corresponds to 375 and $932 \mathrm{kpc}$ for the lowest and largest redshifts in the sample, respectively. The total area covered, including the 24 clusters, is $\sim 135 \mathrm{arcmin}^{2}$. The exposure times of the frames vary between 2000 and $5000 \mathrm{~s}$, reaching average $(5 \sigma)$ limiting $\mathrm{AB}$ magnitudes of $\sim 26$. A summary of the properties of the data set is shown in Table 2 .

\subsection{Spitzer NIR and MIR photometry}

A series of programs with Spitzer have covered all CLASH clusters with IRAC (Fazio et al. 2004) 3.6 and $4.5 \mu \mathrm{m}$ bands. Furthermore, 40 percent of them have also been observed with IRAC 5.8 and

\footnotetext{
${ }^{2} \mathrm{CC}$ clusters are defined as those systems with X-ray cooling times $<1 \mathrm{Gyr}$ (Fabian 1994).

${ }^{3}$ https://archive.stsci.edu/prepds/clash/
} 
Table 1. Description of the galaxy cluster sample. We display the following information: (1) cluster IDs; (2) and (3) coordinates of the cluster centre as in Postman et al. (2012); (4) redshift, Postman et al. (2012); (5) velocity dispersion (we use the value $\sigma_{\mathrm{cl}}=1600 \mathrm{~km} \mathrm{~s}^{-1}$ when no observational estimation was found in the literature); (6) radius within which the mean density is 200 times the critical density at the redshift where the cluster is located [ $\sim R_{\text {virial }}$ according to the simulations of Evrard, Metzler \& Navarro 1996; we use $R_{200}=2000 \mathrm{kpc}$, see for instance Umetsu et al. (2014), for those cases for which no precise value was found in the literature]; (7) the SF activity of the BCG as quantified through the emission of the UV, corrected for extinction $\left(\mathcal{S F} \mathcal{R}_{\mathrm{BCG}, \mathrm{UV} \text {,corr.; }}\right.$; Fogarty et al. 2015), and the emission in the FIR ( $\mathcal{S F} \mathcal{F}_{\mathrm{BCG}, \mathrm{TIR}}$; Rawle et al. 2012a); (8) CC tracer $\mathcal{C}$ parameter as published by Donahue et al. (2016); (9) number of spectroscopic redshifts within the area covered by the CLASH catalogue $\left(\sim 0.0015 \mathrm{deg}^{2}\right)$.

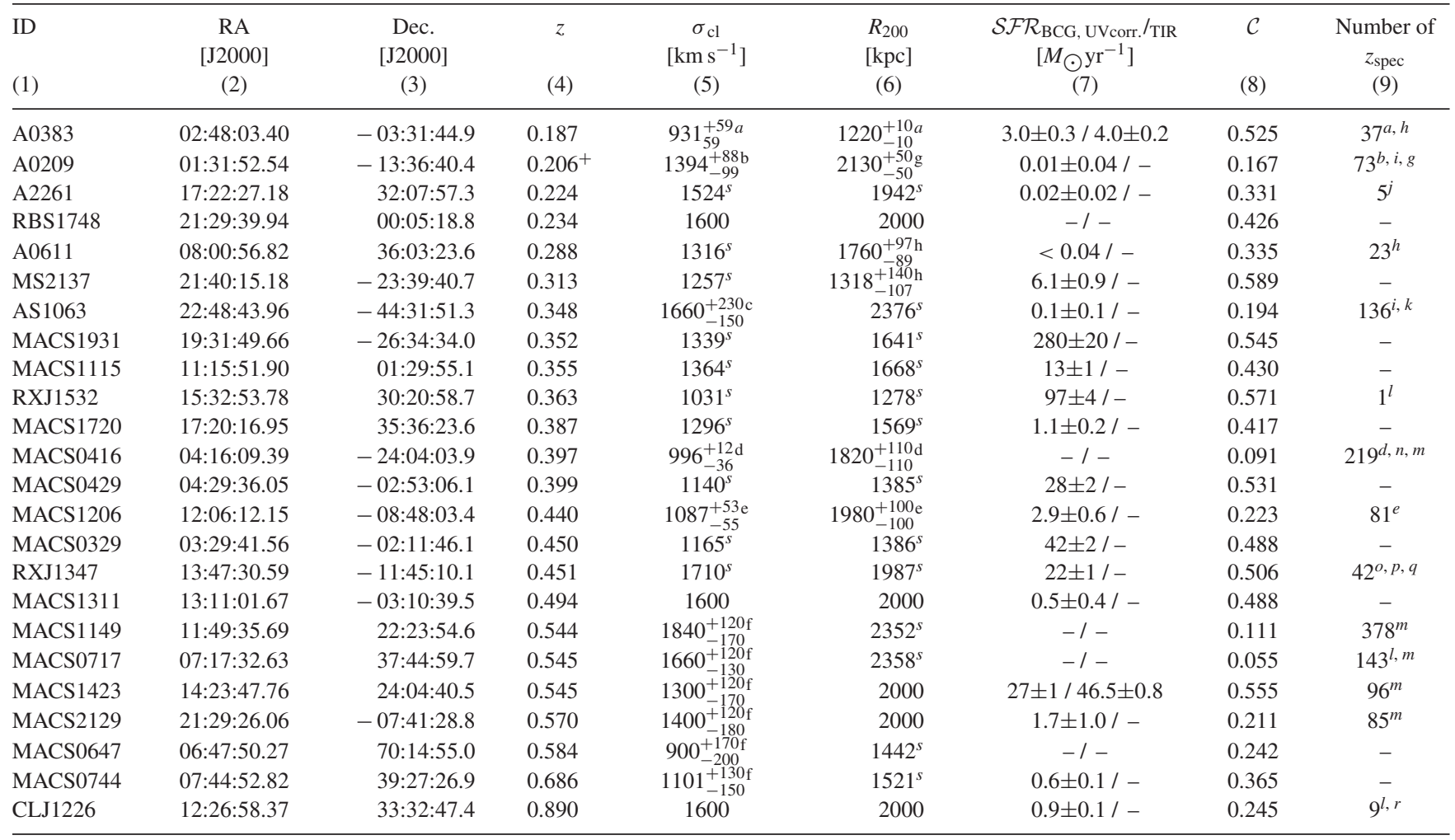

Notes: ${ }^{+} 0.209$ according to Mercurio et al. (2003); ${ }^{a}$ Geller et al. (2014); ${ }^{b}$ Mercurio et al. (2003); ${ }^{c}$ Gómez et al. (2012); ${ }^{d}$ Balestra et al. (2016); ${ }^{e}$ Biviano et al. (2013); ${ }^{f}$ Ebeling et al. (2007); ${ }^{g}$ Annunziatella et al. (2016); ${ }^{h}$ Newman et al. (2013); ${ }^{i}$ Rosati et al. (2014); ${ }^{j}$ Coe et al. (2012); ${ }^{k}$ Karman et al. (2015);

${ }^{l}$ Huchra et al. (2012); ${ }^{m}$ Ebeling, Ma \& Barrett (2014); ${ }^{n}$ Treu et al. (2015) and Schmidt et al. (2014); ${ }^{o}$ Ravindranath \& Ho (2002); ${ }^{p}$ Cohen \& Kneib (2002);

${ }^{q}$ Shectman et al. (1996); ${ }^{r}$ Abazajian et al. (2009); ${ }^{s} \sigma \mathrm{cl}$ and $\mathcal{R}_{200}$ derived using the value of the mass within $\mathcal{R}_{200}\left(\mathcal{M}_{200}\right)$ from Umetsu et al. (2014).

$8.0 \mu \mathrm{m}$ channels, and 50 per cent has been covered by MIPS (Rieke et al. 2004) $24 \mu \mathrm{m}$ band. These data were extracted from the Spitzer Heritage archive ${ }^{4}$. Spitzer images reduction, source detection, and photometry were carried out as described in Pérez-González et al. (2005, 2008), for MIPS and IRAC, respectively. Briefly, the data reduction was carried out with MOPEX (Mosaicking and Pointsource EXtraction), the package provided by the Spitzer Science Center for reducing and analysing imaging data. In the case of IRAC, the source detection and photometry were carried out with SEXTRACTOR (Bertin \& Arnouts 1996), using the same procedure as Huang et al. (2004). Photometry was performed using a small circular aperture, and an aperture correction was applied to get the total flux. IRAC beam sizes are 2.1, 2.1, 2.2, and 2.2 arcsec, respectively, for increasing wavelengths. The average sensitivities reached at $5 \sigma$ are $1.4,1.5,4.5$, and $4.2 \mu \mathrm{Jy}$. In the case of MIPS images, characterized by a larger point spread function (PSF), the photometry was extracted by PSF fitting. Several detection passes are used in order to make catalogues as complete as possible, in spite of the significant source confusion. The MIPS $24 \mu \mathrm{m}$ beam

\footnotetext{
${ }^{4}$ http://irsa.ipac.caltech.edu/applications/Spitzer/SHA
}

size is 5 arcsec. The average MIPS $24 \mu \mathrm{m}$ limiting flux at $5 \sigma$ is $234 \mu \mathrm{Jy}$. In Tables 2 and 3, we summarize the properties of these photometric catalogues. We report the heterogeneous sensitivities reached by IRAC and MIPS imaging on the different CLASH clusters. In particular, MIPS $24 \mu \mathrm{m}$ limiting fluxes vary between 77 and $852 \mu \mathrm{Jy}$.

\subsection{Herschel FIR photometry}

This study employs the PACS 100 and $160 \mu \mathrm{m}$, and SPIRE 250, 350 , and $500 \mu \mathrm{m}$ imaging provided by HLS for all the clusters. We use the catalogues created by the HLS team following the methodology presented by Pérez-González et al. (2010) and Rawle et al. (2010, 2016). Source catalogues and photometry in all bands were obtained with standard PSF fitting methodology, relying on a set of fixed IRAC and MIPS prior position catalogues. PACS imaging at 100 and $160 \mu \mathrm{m}$ has mean $5 \sigma$ flux limits of 4.7 and $8.7 \mathrm{mJy}$, while in the three SPIRE bands, the typical $5 \sigma$ limits are $19.4,15.3$, and $13.7 \mathrm{mJy}$, respectively for the 250,350 , and $500 \mu \mathrm{m}$ bands. The beam sizes for the five Herschel bands (sorted by increasing effective wavelength) are 8, 12, 18, 25, and 36 arcsec, respectively. 
Table 2. In this table, we show an overview of the photometric bands used in this work: (1) name of the instrument and observing band; (2) effective wavelength of the filter; (3) median FWHM of the PSF in arcseconds; (4) name of the project to which the data belongs.

\begin{tabular}{|c|c|c|c|}
\hline $\begin{array}{l}\text { Band } \\
\text { (1) }\end{array}$ & $\begin{array}{c}\lambda_{\text {eff }} \\
(2)\end{array}$ & $\begin{array}{c}\text { FWHM } \\
\text { (arcsec) } \\
(3)\end{array}$ & $\begin{array}{c}\text { Project } \\
\text { (4) }\end{array}$ \\
\hline WFC3-F225W & $237.84 \mathrm{~nm}$ & 0.08 & CLASH \\
\hline WFC3-F275W & $271.47 \mathrm{~nm}$ & 0.08 & CLASH \\
\hline WFC3-F336W & $335.86 \mathrm{~nm}$ & 0.07 & CLASH \\
\hline WFC3-F390W & $393.22 \mathrm{~nm}$ & 0.07 & CLASH \\
\hline ACS-F435W & $436.33 \mathrm{~nm}$ & 0.08 & CLASH \\
\hline ACS-F475W & $475.05 \mathrm{~nm}$ & 0.08 & CLASH \\
\hline ACS-F606W & $596.11 \mathrm{~nm}$ & 0.08 & CLASH \\
\hline ACS-F625W & $630.97 \mathrm{~nm}$ & 0.08 & CLASH \\
\hline ACS-F775W & $770.59 \mathrm{~nm}$ & 0.08 & CLASH \\
\hline ACS-F814W & $807.31 \mathrm{~nm}$ & 0.09 & CLASH \\
\hline ACS-F850LP & $905.26 \mathrm{~nm}$ & 0.09 & CLASH \\
\hline WFC3-F105W & $1.06 \mu \mathrm{m}$ & 0.13 & CLASH \\
\hline WFC3-F110W & $1.15 \mu \mathrm{m}$ & 0.13 & CLASH \\
\hline WFC3-F125W & $1.25 \mu \mathrm{m}$ & 0.14 & CLASH \\
\hline WFC3-F140W & $1.40 \mu \mathrm{m}$ & 0.14 & CLASH \\
\hline WFC3-F160W & $1.54 \mu \mathrm{m}$ & 0.15 & CLASH \\
\hline IRAC $-3.6 \mu \mathrm{m}$ & $3.56 \mu \mathrm{m}$ & 2.1 & a \\
\hline IRAC $-4.5 \mu \mathrm{m}$ & $4.50 \mu \mathrm{m}$ & 2.1 & a \\
\hline IRAC $-5.8 \mu \mathrm{m}$ & $5.74 \mu \mathrm{m}$ & 2.2 & $\mathrm{a}$ \\
\hline IRAC $-8.0 \mu \mathrm{m}$ & $7.93 \mu \mathrm{m}$ & 2.2 & a \\
\hline MIPS-24 $\mu \mathrm{m}$ & $23.84 \mu \mathrm{m}$ & 5 & b \\
\hline PACS $-100 \mu \mathrm{m}$ & $102.25 \mu \mathrm{m}$ & 8 & HLS \\
\hline PACS $-160 \mu \mathrm{m}$ & $165.59 \mu \mathrm{m}$ & 12 & HLS \\
\hline SPIRE $-250 \mu \mathrm{m}$ & $253.13 \mu \mathrm{m}$ & 18 & HLS \\
\hline SPIRE $-350 \mu \mathrm{m}$ & $355.87 \mu \mathrm{m}$ & 25 & HLS \\
\hline SPIRE $-500 \mu \mathrm{m}$ & $511.19 \mu \mathrm{m}$ & 36 & HLS \\
\hline
\end{tabular}

Notes: ${ }^{a}$ Spitzer Programs numbers 17 (PI: Fazio), 83 (PI: Rieke), 545 (PI: Egami), 40652 (PI: Kocevski), 50393 (PI: Kocevski), 60034 (PI: Egami), and 80168 (PI: Bouwens). ${ }^{b}$ Spitzer Programs numbers 83 (PI: Rieke), 40652 (PI: Kocevski), 40872 (PI: Smith), and 50393 (PI: Kocevski).

\subsection{Spectroscopic data}

One of the programs with a greater contribution to our spectroscopic redshift sample is the spectroscopic survey carried out on the 13 southern CLASH clusters with the Visible Multi-Object Spectrograph (VIMOS; Le Fèvre et al. 2003) mounted on the Very Large Telescope (VLT), the so-called CLASH-VLT survey (CLASH-VLT Large Programme 186.A0.798; PI: P. Rosati; Rosati et al. 2014). We refer the reader to Biviano et al. (2013) and Balestra et al. (2016) for details on spectroscopic data, target selection, and performance statistics of the mentioned project. We also make use of spectroscopic redshift measurements from the Grism Lens Amplified Survey from Space (GLASS; Schmidt et al. 2014; Treu et al. 2015), a large HST program aimed at obtaining grism spectroscopy of the HFF. Besides these, we also gather spectroscopic redshifts from other surveys (see Table 1 for a complete list of the works included). Finally, we also retrieve redshifts through NASA/IPAD Extragalactic Database, mainly from the 2MASS Redshift Survey (Huchra et al. 2012), and the Seventh Data Release of the Sloan Digital Sky Survey (Abazajian et al. 2009). In Section 4, we describe the properties of the final spectroscopic sample.

\section{MULTIWAVELENGTH PHOTOMETRY}

We merge the photometric data sets described in the previous section to obtain UV-to-FIR SEDs for all the sources in the catalogues released by CLASH. To this end, we use the RAINBOW Cosmological Database (Pérez-González et al. 2008, Barro et al. 2011a,b) and associated software package. We use CLASH catalogues as parent catalogues to take advantage of the high resolution of HST imaging. However, this requires taking special care of the inevitable blending of sources in bands with poorer resolution, as well as possible counterpart misidentification.

In the following subsections, we describe the strategy that we use for the build-up of our multiwavelength photometric catalogue.

\subsection{Cross-matching catalogues}

Initially, RAINBOW searches for counterparts of our parent catalogue in the rest of the bands. In practice, each catalogue is cross-matched to the CLASH positions. RAINBOW takes into account possible astrometry offsets between the bands by re-aligning each pair of them using the positions of several sources in small $1 \operatorname{arcmin} \times$ 1 arcmin boxes around a given source. The search radii we use to find counterparts candidates are 1.5, 2.5, 2.5, 4.0, 9.0, 9.0, and 12.0 arcsec for IRAC, MIPS $24 \mu \mathrm{m}$, PACS 100 and $160 \mu \mathrm{m}$, and SPIRE 250, 350, and $500 \mu \mathrm{m}$ catalogues. These values are chosen in order to cope with the typical WCS offsets between different images, as well as uncertainties in the determination of the centre for faint MIPS and Herschel sources. We note, however, that a comparison of the CLASH versus MIPS/Herschel coordinates for secure (i.e. bright) $\mathrm{M}$ - and FIR sources points out that the typical WCS uncertainty is $\sim 0.2 \operatorname{arcsec}$ for IRAC, $\sim 0.4 \operatorname{arcsec}$ for MIPS, $\sim 0.4$ arcsec for PACS, and $\sim 1.3 \operatorname{arcsec}$ for SPIRE. In Section 3.3, we take into account both the search radius and the WCS accuracy measurements to discuss how many HST counterparts we find for each MIR and FIR source, and how we select the most likely among the former.

\subsection{IRAC fluxes deblending}

The IRAC photometry is recomputed on CLASH positions following a deconvolution method detailed in Barro et al. (2011a). The procedure is similar to that used in, e.g. Grazian et al. (2006), Wuyts et al. (2008), Williams et al. (2009), or Wang et al. (2010), and briefly consists on the convolution of the PSF of the higher resolution image to the IRAC PSF and a subsequent scaling of the flux of each source in a way that the total flux equals the emission of the blended source in the lower resolution image.

\subsection{MIR and FIR counterpart assignment}

Given the larger beam sizes of the MIR/FIR bands, a simple crosscorrelation of the optical/NIR and MIR/FIR catalogues frequently assigns the same MIR/FIR sources to different optical/NIR counterparts (especially when using HST images). On average, the relaxed search radii we use to cross-match catalogues lead to the assignation of each MIPS $24 \mu \mathrm{m}$, PACS, and SPIRE source to 2, 5, and 32 optical/NIR sources, respectively. However, within the WCS accuracy measurements there are, on average, one optical/NIR source for each detection in MIPS $24 \mu \mathrm{m}$, PACS, and SPIRE 250 and $250 \mu \mathrm{m}$, and two optical/NIR sources for each SPIRE $500 \mu \mathrm{m}$ source. These latter values are more informative of the level of uncertainty in 
Table 3. Limiting fluxes $(5 \sigma)$ of the Spitzer and Herschel photometric catalogues used in this work.

\begin{tabular}{|c|c|c|c|c|c|c|c|c|c|c|}
\hline \multirow[b]{2}{*}{$\begin{array}{l}\text { Cluster } \\
\text { (1) }\end{array}$} & \multicolumn{5}{|c|}{$\mathcal{F}_{\lim }(\mu \mathrm{Jy})$} & \multicolumn{5}{|c|}{$\mathcal{F}_{\lim }(\mathrm{mJy})$} \\
\hline & $\begin{array}{c}3.6 \mu \mathrm{m} \\
(2)\end{array}$ & $\begin{array}{c}4.5 \mu \mathrm{m} \\
\text { (3) }\end{array}$ & $\begin{array}{l}5.8 \mu \mathrm{m} \\
\quad(4)\end{array}$ & $\begin{array}{c}8.0 \mu \mathrm{m} \\
(5)\end{array}$ & $\begin{array}{c}\text { Spitzer/MIPS } \\
24 \mu \mathrm{m} \\
\text { (6) }\end{array}$ & $\begin{array}{c}100 \mu \mathrm{m} \\
\text { (7) }\end{array}$ & $\begin{array}{c}160 \mu \mathrm{m} \\
(8)\end{array}$ & $\begin{array}{c}250 \mu \mathrm{m} \\
\text { (9) }\end{array}$ & $\begin{array}{c}350 \mu \mathrm{m} \\
(10)\end{array}$ & $\begin{array}{l}500 \mu \mathrm{m} \\
\quad(11)\end{array}$ \\
\hline A0383 & 2.7 & 2.2 & 6.7 & 6.3 & 317.6 & 4.8 & 9.4 & 14.8 & 13.7 & 10.8 \\
\hline A0209 & 2.0 & 1.7 & 5.2 & 5.4 & 268.7 & 4.6 & 9.1 & 14.6 & 14.0 & 10.7 \\
\hline A2261 & 1.9 & 1.9 & 5.8 & 4.6 & 108.5 & 4.6 & 8.9 & 20.0 & 16.1 & 14.0 \\
\hline MS2137 & 1.8 & 1.6 & 7.1 & 7.7 & 97.7 & 5.1 & 9.4 & 14.5 & 13.3 & 11.1 \\
\hline AS1063 & 2.2 & 1.7 & 6.6 & 6.0 & 76.9 & 4.8 & 7.7 & 14.7 & 14.6 & 10.9 \\
\hline MACS1931 & 3.6 & 2.7 & - & - & 851.9 & 4.5 & 8.7 & 19.5 & 15.2 & 13.4 \\
\hline MACS1115 & 1.3 & 1.4 & - & - & - & 4.7 & 8.7 & 20.4 & 16.1 & 14.5 \\
\hline RXJ1532 & 1.2 & 1.2 & - & - & 180.3 & 4.8 & 8.4 & 18.3 & 14.5 & 13.5 \\
\hline MACS 1720 & 0.9 & 0.8 & - & - & - & 4.7 & 8.7 & 19.6 & 14.9 & 13.0 \\
\hline RXJ1347 & 1.7 & 1.5 & 4.5 & 2.7 & 143.7 & 4.3 & 7.8 & 21.1 & 18.6 & 18.5 \\
\hline MACS1311 & 1.2 & 1.4 & - & - & - & 4.7 & 8.4 & 20.1 & 15.6 & 14.2 \\
\hline MACS1149 & 0.9 & 0.9 & - & - & - & 4.7 & 8.6 & 15.1 & 15.3 & 14.9 \\
\hline MACS0717 & 1.7 & 1.9 & - & - & 133.3 & 4.7 & 9.2 & 17.8 & 15.9 & 12.0 \\
\hline MACS 1423 & 1.4 & 1.8 & - & - & 95.5 & 5.2 & 9.5 & 14.2 & 12.6 & 10.3 \\
\hline MACS2129 & 1.9 & 1.7 & 1.1 & 1.6 & 112.6 & 5.2 & 13.7 & 33.5 & 28.3 & 29.2 \\
\hline MACS0647 & 1.1 & 1.3 & - & - & - & 4.8 & 10.8 & 23.1 & 20.3 & 14.7 \\
\hline MACS0744 & 2.2 & 3.0 & 1.2 & 1.8 & - & 4.4 & 8.4 & 14.5 & 14.1 & 11.3 \\
\hline CLJ1226 & 3.6 & 3.6 & 2.0 & 1.8 & 131.7 & 6.5 & 11.3 & 22.2 & 18.3 & 18.6 \\
\hline
\end{tabular}

our cross-matching procedure and reliability of the counterparts identification, as well as possible blending affecting the lowresolution bands.

Due to the large difference between the resolution of CLASH and MIR/FIR bands, it is not advisable to apply a deblending procedure such as it was done on IRAC photometry. Instead, we limit our approach to the identification of the most likely counterpart, or dominant contributor to the MIR/FIR fluxes, among the multiple short-wavelength counterparts assigned to the same MIR/FIR sources. The fact that the FIR catalogues are built using IRAC and MIPS $24 \mu \mathrm{m}$ priors guarantees a consistent framework to link the photometry across the whole wavelength range. Different studies have addressed the task of identifying counterparts of FIR/submillimetre galaxies in shorter wavelengths (e.g. Alberts et al. 2013), avoiding using simply the shortest distance match with the aim of achieving a more physically driven identification. Our approach steps through the NIR-to-FIR wavelength range and evaluates which of the IR SEDs of the multiple candidates is most likely to be associated with the MIR/FIR detection.

We first set local and average signal-to-noise (S/N) limits in the FIR bands. These limits are $2 \sigma$ and $3 \sigma$ for MIPS and Herschel bands (see Table 3, where we show the flux values corresponding to the $5 \sigma$ detection in each band and cluster). The $2 \sigma$ is used to maximize the information available to identify the FIR counterparts, however, we clarify that we do not consider MIPS $24 \mu \mathrm{m}$ fluxes below $3 \sigma$ detections in the rest of the work. Then, we select as the optical/NIR counterpart of each MIPS $24 \mu \mathrm{m}$ source the brightest candidate in the reddest IRAC band available. Then, we shift this methodology to larger wavelength bands. We select as the optical/NIR counterpart of each PACS source the brightest candidate in MIPS $24 \mu \mathrm{m}$. When MIPS is not available, we use the reddest IRAC band in which the source is detected. Finally, we select as the optical/NIR counterpart of each SPIRE source, the brightest candidate in the reddest PACS band available, if any. Otherwise, MIPS $24 \mu \mathrm{m}$ and IRAC bands are used. If different optical/NIR candidates present very similar fluxes (within $1 \sigma$ ) in the band that is used to identify the counterpart, we impose a criterion of minimum distance, and therefore, we select as the optical/NIR counterpart the galaxy with the closest position to the MIR/FIR source. In all cases described, the MIPS, PACS, and SPIRE fluxes of the CLASH sources that are not identified as real counterparts are flagged and they are not used subsequently. Therefore, each MIR/FIR source is assigned to a single optical/NIR source. We note that using IRAC as a tracer of PACS or SPIRE emitters can lead to spurious associations. This is because NIR and FIR trace different components and processes in the galaxies. In the clusters with MIPS coverage, the average fraction of Herschel sources' optical counterparts identified by their IRAC fluxes is 20 percent and 32 percent for PACS and SPIRE, respectively. These values increase, however, in those fields without MIPS photometry, reaching 91 per cent and 49 per cent, respectively. These cases are flagged for further check. After a thorough visual inspection of the output of our procedure, we detect only obvious mismatch cases in galaxies located in the border of the HST/WFC3 images. We have identified a number of galaxies suffering from overdeblending in the CLASH catalogues, which means that the photometry of these galaxies are divided into different sources. In these cases, the flux of the MIR and FIR catalogues are generally assigned to source corresponding to the central region of the galaxy.

\section{PHOTOMETRIC REDSHIFTS}

Photometric redshifts $\left(z_{\text {phot }}\right)$ are computed using the EAZY code (Brammer, van Dokkum \& Coppi 2008), specifically conceived 
for this task. EAZY is a template-fitting code based on $\chi^{2}$ minimization between observed photometry and a set of six SED templates. Among them, five templates are generated following the Blanton \& Roweis (2007) non-negative matrix factorization algorithm with PEGASE stellar population synthesis models (Fioc \& Rocca-Volmerange 1997) and a calibration set of synthetic photometry derived from semi-analytic models. The last one is a dusty starburst model, and it is added to the set in order to compensate for the lack of dusty galaxies in the calibration photometric sample.

The achievable quality of photometric redshifts depends strongly on the quality of the photometric data set itself, and the wavelength domain it covers (e.g. Pacifici et al. 2012). In particular, it benefits from high-quality photometry sampling strong continuum features (e.g. Lyman or Balmer breaks). In this sense, the $16 \mathrm{CLASH}$ broadband photometric points enable high levels of accuracy in the photometric redshift estimation (Jouvel et al. 2014, Molino et al. 2017, Connor et al. 2017). In order to make use of the whole potential of our data set, we fit not only the whole wavelength range covered by CLASH, but also the IRAC photometric points. Furthermore, for those clusters with available spectroscopic samples, we perform a zero-point fine-tuning (following the methodology by Barro et al. 2011a,b) to account for mismatches between the CLASH colours and the SED-fitting template library colours, or other hypothetical systematic problems. The median absolute zeropoints used are 3 percent and 5 percent for CLASH and IRAC bands, respectively.

\subsection{Photometric redshifts quality}

We assess the quality of the $z_{\text {phot }}$ obtained for each cluster by comparing them against the available and reliable ${ }^{5} z_{\text {spec }}$. We crosscorrelate CLASH data set with the spectroscopic catalogues using a radius of 0.5 arcsec. The total reference spectroscopic sample is composed of 1034 spectroscopically confirmed galaxies within the area of the WFC3 imaging (i.e. the area covered by the photometric catalogues) over the 24 CLASH+HLS clusters we analyse. This sample is by definition inhomogeneous, as can be expected of the combination of studies designed with different scientific objectives and selection criteria. It extends between $0.1<z \lesssim 9$, with the 90 per cent of the galaxies at $z<2$. Fig. 1 displays the distribution of $z_{\text {spec }}$ (empty histogram), and the distribution of magnitudes in the ACS/F814W band (empty histogram; nested panel).

A number of quantities have been used in the literature to quantify the behaviour of the data points in this diagram (see e.g. Pelló et al. 2009), either in terms of scatter, as well as the presence of outliers and systematic offsets. In the last decade, the normalized median absolute deviation $\left(\sigma_{\text {NMAD }}\right.$; Hoaglin, Mosteller \& Tukey $1983)$ of the difference between the $z_{\text {phot }}$ and the $z_{\text {spec }}\left(\Delta z=z_{\text {phot }}\right.$ $\left.-z_{\text {spec }}\right)$ has been frequently used to characterize the scatter of the distribution of $z_{\text {phot }}$ (e.g. Ilbert et al. 2009). A typical photometric redshift error distribution has tails that clearly depart from a pure Gaussian distribution, in addition to a relatively large fraction of outliers. The $\sigma_{\mathrm{NMAD}}$ estimator manages to achieve a stable estimate of the spread of the core of the $z_{\text {phot }}$ distribution without being

\footnotetext{
${ }^{5}$ The reliability of the $z_{\text {spec }}$ is given by the spectroscopic surveys in the form of a quality flag normally linked to the number and $\mathrm{S} / \mathrm{N}$ of the spectral features identified on the spectrum, that are used to calculate the redshift.
}

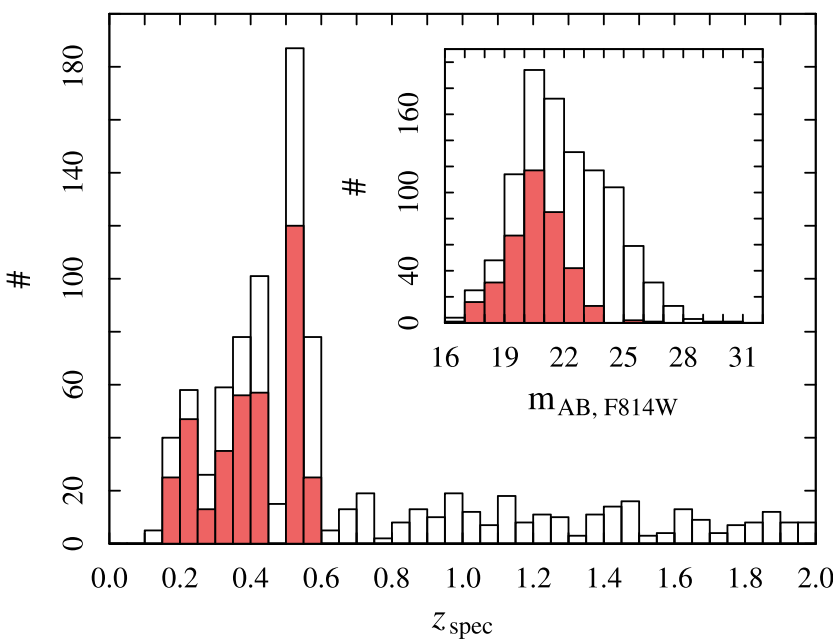

Figure 1 Distribution of $z_{\text {spec }}$ for our spectroscopic sample (1034 galaxies; empty histogram). The distribution of the redshifts of the 378 spectroscopically confirmed cluster members is given in red. In this figure, we show the distribution up to $z=2$, which contains 90 percent of the sample. The nested panel shows the corresponding distribution of magnitudes in the ACS/F814W band.

affected by the mentioned tails. It is defined as

$\sigma_{\mathrm{NMAD}}=1.48 \times \operatorname{median}\left(\frac{|\Delta z-\operatorname{median}(\Delta z)|}{1+z_{\mathrm{spec}}}\right)$.

Following the notation by Barro et al. (2011b), we consider the fraction of catastrophic outliers, $\eta$, defined as those cases for which

$|\Delta z| /\left(1+z_{\text {spec }}\right)>0.2$.

Finally, in order to characterize the systematic offsets of the photometric redshifts obtained, $\delta$, we use the expression

$\delta=\Delta z /\left(1+z_{\text {spec }}\right)$.

When compared with the spectroscopic sample, our photometric redshift estimations present $\sigma_{\mathrm{NMAD}}=0.04$, and 8 percent of catastrophic outliers (see Fig. 2). The outliers are typically either faint sources with noisy photometry in HST and/or IRAC bands (e.g. high-redshift galaxies, objects located in the border of the CLASH catalogues) or galaxies for which the IRAC photometry seems to be contaminated by bright nearby objects. We do not identify systematic effects, with an average $\delta=-0.01$. These values are comparable with those published by Jouvel et al. (2014) for CLASH clusters.

As we are using the $z_{\text {phot }}$ to select cluster members, we also assess their quality using only a subsample of spectroscopic members. We follow the selection criteria used by Molino et al. (2017, see Section 4.2) in order to be able to compare our results with theirs. The cluster member's reference spectroscopic sample is formed by galaxies for which the difference between its $z_{\text {spec }}$ and the cluster redshift $\left(\Delta z_{\mathrm{cl}}\right)$ fulfills $\left|\Delta z_{\mathrm{cl}}\right| \leq 0.01$. Also, in order to guarantee an optimal sampling of the optical and NIR SED, only galaxies detected at least on 14 CLASH bands are considered. Using these criteria, we select 378 galaxies (see red histogram in Fig. 1). In this case, our photometric redshift estimations present $\sigma_{\mathrm{NMAD}}=0.03$, and 2 per cent of catastrophic outliers. These values are comparable with to those obtained by Molino et al. (2017): $\sigma_{\mathrm{NMAD}}=0.02$, and 


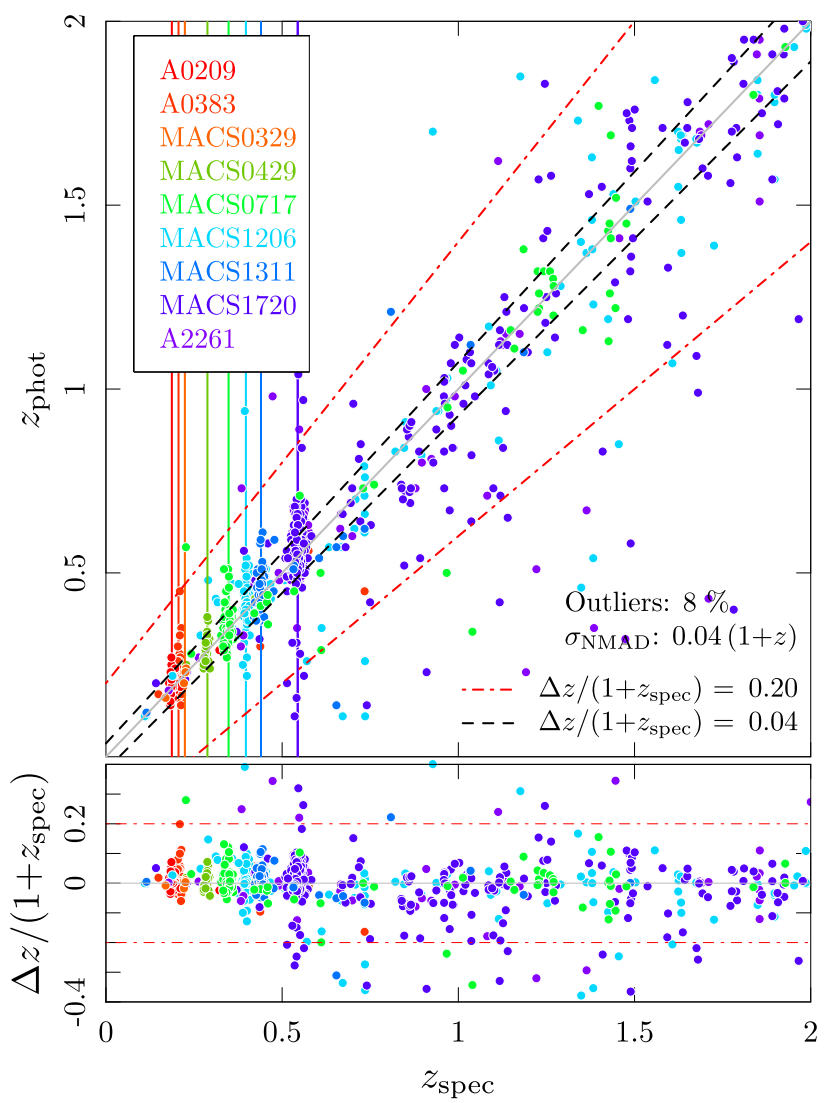

Figure 2 Evaluation of the $z_{\text {phot }}$ quality. The black and red dashed lines show, respectively, the accuracy reached by our results considering the whole spectroscopic sample and the definition of outlier. The vertical lines mark the redshift of each cluster (Table 2).

$\eta<3$ percent. Neither in this case we identify systematic effects, with an average deviation $\delta=0.01$.

\section{SPECTRAL ENERGY DISTRIBUTION FITTING WITH RAINBOW}

In order to derive the physical properties of the galaxies found on CLASH+HLS fields, we apply a SED-fitting analysis to the entire data set gathered and described in the previous sections. We use the RAINBOW Cosmological Database software package (PérezGonzález et al. 2008; Barro et al. 2011a,b) to fit, on the one hand, the optical/NIR photometry (CLASH and Spitzer/IRAC), and on the other hand, the MIR/FIR photometry (Spitzer/MIPS and Herschel). In both cases, we fix the redshifts derived with EAZY or, when available, the $z_{\text {spec }}$.

In particular, the optical/NIR fitting code performs a $\chi^{2}$ minimization between the observed data and a set of semi-empirical template SEDs computed from spectroscopically confirmed galaxies modelled with PEGASE stellar population synthesis models (Fioc \& Rocca-Volmerange 1997). In particular, we use the templates generated by Pérez-González et al. 2008 (see their Appendix B) assuming a single stellar population with a exponentially declining star formation history $\left(\operatorname{SFH} ; \mathcal{S F R}(t) \propto \mathrm{e}^{-t / \tau}\right)$ with a time-scale $(\tau)$ varying between $1 \mathrm{Myr}$ (instantaneous burst) and $100 \mathrm{Gyr}$ (constant $\mathrm{SFH}$ ) and an age that can take values between $1 \mathrm{Myr}$ and $13.5 \mathrm{Gyr}$. We also assume a Salpeter (1955) IMF spanning stellar masses from
0.1 to $100 M_{\odot}$, metallicity $(\mathcal{Z})$ values $0.005,0.0 .02,0.2,0.4,1.0$, 2.5, and $5.0 \mathcal{Z}_{\odot}$, extinction between 0 and $5 \mathrm{mag}$, and a Calzetti et al. (2000) attenuation law. We complement the set of templates with active galactic nucleus (AGN) empirical templates drawn from Polletta et al. (2007) that account for the galaxies whose UV-to-NIR emission is dominated by an AGN. In the case of the MIR/FIR SED fitting, the $\chi^{2}$ minimization is performed between the observed photometry and the typical dust emission models by Chary \& Elbaz (2001), Dale \& Helou (2002), Rieke et al. (2009), and Draine \& Li (2007).

\subsection{Stellar masses}

The $\mathcal{M}_{*}$ of each galaxy is estimated by RAINBOW from the average scale factor required to match the template monochromatic luminosities to the observed fluxes, weighted with the photometric errors. The random uncertainty of the $\mathcal{M}_{*}$ is derived from the dispersion in the mass-luminosity ratio in the different bands. The average expected uncertainty in the estimations of $\mathcal{M}_{*}$ taking into account variations in $\mathcal{Z}, \mathrm{SFH}$, or IMF are within 0.3 dex (PérezGonzález et al. 2008).

\subsection{Star formation rates}

We take advantage of our rich data set to analyse the SF activity undergone by the galaxies in these fields in terms of total $\mathcal{S F R}$ $\left(\mathcal{S F R} \mathcal{R}_{\mathrm{TOT}}\right)$. Similarly to previous works (see Kennicutt \& Evans 2012 and references therein), we consider that the total SF activity of a galaxy can be derived from the combination of (1) the UV luminosity emitted by young stars that is able to escape from the ISM, and (2) the UV luminosity that is absorbed by the ISM and re-emitted in the MIR/FIR regime. We use the recipe of Bell et al. (2005), which is based on the calibration of Kennicutt (1998):

$$
\mathcal{S F \mathcal { R } _ { \mathrm { TOT } }}=\mathcal{S F \mathcal { R }} \mathcal{T I R}+\mathcal{S F} \mathcal{R}_{\mathrm{UV}}
$$

$\mathcal{S F R}_{\mathrm{TIR}} / M_{\odot} \mathrm{yr}^{-1}=1.8 \times 10^{-10} \mathcal{L}_{\mathrm{TIR}} / L_{\odot}$

$$
\mathcal{S} \mathcal{F} \mathcal{R}_{\mathrm{UV}} / M_{\odot} \mathrm{yr}^{-1}=5.9 \times 10^{-10} \mathcal{L}_{2800 \AA} / L_{\odot}
$$

where $\mathcal{L}_{\mathrm{TIR}}$ is the integrated total IR luminosity and $\mathcal{L}_{2800}$ is the rest-frame monochromatic luminosity at $2800 \AA$ (uncorrected for extinction).

We compute $\mathcal{L}_{\mathrm{TIR}}$ by integrating the best-fitting Draine \& $\mathrm{Li}$ (2007) dust emission templates between 8 and $1000 \mu \mathrm{m}$. As we mentioned previously, we use four different libraries of dust emission models in our analysis. The main differences between these models are the prominence of the polycyclic aromatic hydrocarbons and their dependence with the total IR luminosity, as well as the ratio between the mass of hot and cold dusts. A discussion on these properties is beyond the scope of this paper, nevertheless, we use all these template sets to include the differences between the assumptions made by them in the uncertainty of the total IR luminosity. Therefore, the $\mathcal{L}_{\mathrm{TIR}}$ values given in this work are derived from the Draine \& Li (2007) libraries, whereas the uncertainties are the rms of the $\mathcal{L}_{\text {TIR }}$ estimations using the four template libraries. We have checked that the differences between the luminosities given by the best-fitting templates of each library are of the order of $\lesssim 20$ per cent.

We calculate $\mathcal{L}_{2800}$ interpolating the best-fitted optical/NIR empirical template at $2800 \AA$ (rest frame). This wavelength is covered by observational data over the whole redshift range of interest. 
Obviously, this formalism can only be used in the case of galaxies detected in the MIR/FIR. For those galaxies not detected by MIPS or Herschel, we compute $\mathcal{S} \mathcal{F} \mathcal{R}_{\text {TOT }}$ by correcting the UV luminosities (i.e. $\left.\mathcal{S} \mathcal{F} \mathcal{R}_{\mathrm{UV}}\right)$ for dust attenuation $\left(\mathcal{A}_{\mathrm{UV}}\right)$ following the expression

$\mathcal{S F} \mathcal{R}_{\mathrm{TOT}}=\mathcal{S F} \mathcal{R}_{\mathrm{UV}, \text { corr. }}=\mathcal{S} \mathcal{F} \mathcal{R}_{\mathrm{UV}} \times 10^{0.4 \mathcal{A}_{\mathrm{UV}}}$

where the $\mathcal{S} \mathcal{F} \mathcal{R}_{\mathrm{UV}}$ is obtained using equation (6).

Meurer, Heckman \& Calzetti (1999) demonstrate that local starburst galaxies exhibit a relatively tight, monotonic relation between the ratio between the UV and the TIR luminosity $(\mathcal{I} \mathcal{X})$ and the UV slope $\left(\beta^{6}\right)$. Through this relationship, they derive a relation between the extinction of the UV (in particular, the attenuation at $1600 \AA$ ) and the $\beta$ itself, providing a simple relation that can be applied to correct UV luminosities. However, this and other typical attenuation recipes based on the UV slope (e.g. Calzetti et al. 1994) are derived for extreme starburst galaxies, while the sources for which we need the correction (i.e. those not-detected in the MIR/FIR) are less extreme SFGs. Thus, using those expressions can lead to an overestimation of the extinction and an overcorrection of the UV luminosity. Therefore, we derive an extinction correction optimized for our work (see Appendix B).

In what follows, the values of the $\mathcal{S} \mathcal{F} \mathcal{R}_{\mathrm{TOT}}$ refer to the $\mathcal{S} \mathcal{F} \mathcal{R}_{\mathrm{UV} \text {,corr. }}$ [equation (7), in which we use our own $\mathcal{A}_{\mathrm{UV}}$ ], except in those cases when the MIR/FIR is available, where we consider the addition of the $\mathcal{S} \mathcal{F} \mathcal{R}_{\mathrm{TIR}}$ and the $\mathcal{S} \mathcal{F} \mathcal{R}_{\mathrm{UV}}$ (equation 4).

\section{CLUSTER MEMBERS SELECTION}

The most unambiguous way to identify cluster members relies on accurate spectroscopic redshifts. However, the acquisition of complete $z_{\text {spec }}$ samples remains infeasible except for a relatively small and bright fraction of the galaxy population. Indeed, using photometric redshifts to estimate the distances to galaxies has become a fundamental aim of galaxy surveys conducted during recent years (e.g. Ilbert et al. 2009; Barro et al. 2011b). Although less accurate than spectroscopic ones, photometric redshifts provide a way to estimate distances for galaxies too faint for spectroscopy or samples too large to be practical for complete spectroscopic coverage. Given the incomplete and inhomogeneous spectroscopic coverage of our sample of clusters, we are forced to use criteria to select cluster members based either on $z_{\text {spec }}$ or $z_{\text {phot }}$.

The spectroscopic cluster members are identified as those galaxies with $z_{\text {spec }}$ within the redshift range defined by the redshift of the cluster, $z_{\mathrm{cl}}$, and its velocity dispersion, $\sigma_{\mathrm{cl}}$. In Table 1 we show the values we use and the corresponding references. In practice, we use the following criteria (see Cava et al. 2009):

$\left|z_{\mathrm{cl}}-z_{\mathrm{spec}}\right|<3 \times \sigma_{\mathrm{cl}} \times\left(1+z_{\mathrm{cl}}\right)$

For those cases in which a $z_{\text {spec }}$ is not available, our member selection relies on the redshift probability distribution, $\mathcal{P}(z)$, given by EAZY instead on the individual $z_{\text {phot }}$ associated to each galaxy. This approach captures all the photometric redshift information, which can significantly reduce the impact of the catastrophic errors in the $z_{\text {phot }}-z_{\text {spec }}$ plane (e.g. Fernández-Soto et al. 2002). This is of key importance to our work, as it translates into a smaller contamination with foreground and background sources

${ }^{6}$ The UV continuum slope is defined by assuming that the UV regime of the SED of a galaxy can be described by a power law $\left(\propto \lambda^{\beta}\right.$, Calzetti, Kinney \& Storchi-Bergmann 1994; Meurer et al. 1999).
Table 4. Summary of some of the quantities used for the identification of cluster members and an evaluation of the technique: (1) cluster IDs; (2) number of spectroscopic members as defined by equation (8); (3) $\sigma_{\text {NMAD }}$ derived for the individual clusters; (4) number of $\sigma_{\text {NMAD }}$ to be used in the integration of the $\mathcal{P}(z)$; (5) membership probability threshold; (6) completeness level (per cent); and (7) fraction of interlopers (per cent).

\begin{tabular}{lcccccc}
\hline ID & $\begin{array}{c}\text { Nuumber } \\
\text { of } z \\
(1)\end{array}$ & $\begin{array}{c}\sigma_{\text {NMAD }} \\
(2)\end{array}$ & $\begin{array}{c}n \\
(4)\end{array}$ & $\begin{array}{c}\mathcal{P}_{\text {thr }} \\
(5)\end{array}$ & $\begin{array}{c}\mathcal{K} \\
(6)\end{array}$ & $\begin{array}{c}\mathcal{I} \\
(7)\end{array}$ \\
\hline A0383 & 33 & 0.02 & 3 & 0.30 & 91 & 8 \\
A0209 & 50 & 0.04 & 3 & 0.75 & 92 & 7 \\
A0611 & 21 & 0.03 & 3 & 0.55 & 95 & 5 \\
AS1063 & 71 & 0.06 & 1 & 0.15 & 87 & 10 \\
MACS0416 & 84 & 0.09 & 2 & 0.75 & 86 & 13 \\
MACS1206 & 51 & 0.06 & 3 & 0.85 & 88 & 11 \\
RXJ1347 & 13 & 0.07 & 1 & 0.25 & 85 & 13 \\
MACS1149 & 160 & 0.12 & 2 & 0.85 & 91 & 9 \\
MACS0717 & 83 & 0.05 & 3 & 0.75 & 89 & 10 \\
MACS2129 & 11 & 0.09 & 1 & 0.70 & 64 & 27 \\
\hline
\end{tabular}

in our cluster member's selection. In particular, we use the method developed by Pelló et al. (2009) based exclusively on photometric redshift estimates. This approach modifies the technique presented by Brunner \& Lubin (2000) in order to take advantage of the $\mathcal{P}(z)$. It calculates a probability of being a cluster member $\left(\mathcal{P}_{\text {member }}\right)$ integrating $\mathcal{P}(z)$ within a redshift range centred in the redshift of the cluster $z_{\mathrm{cl}}$ and with a width $(\Delta z)$ related to the accuracy of the photometric redshifts (see Section 4.1).

$\mathcal{P}_{\text {member }}=\int_{z_{\mathrm{cl}}-\Delta z}^{z_{\mathrm{cl}}+\Delta z} \mathcal{P}(z) \mathrm{d} z$

In our case, we use $\Delta z=n \times \sigma_{\mathrm{NMAD}} \times\left(1+z_{\mathrm{cl}}\right)$. Applying this technique to those galaxies for which we have a reliable spectroscopic redshift, we can calibrate the cluster member selection, which means to find a probability threshold $\left(\mathcal{P}_{\text {thr }}\right)$ over which a galaxy is considered to be a cluster member, given a certain $n$. Table 4 shows the values of $n$ and $\mathcal{P}_{\text {thr }}$ we find to maximize the completeness level $(\mathcal{K})$ and minimize the percentage of interlopers $(\mathcal{I})$ for those clusters with spectroscopic members. Table 4 also gives the values of $\mathcal{K}$ and $\mathcal{I}$ for each case. We reach $\mathcal{K}>80$ per cent and $\mathcal{I}<20$ per cent (limiting values used also by Pelló et al. 2009) for 9 out of the 10 clusters with more than 10 spectroscopic cluster members available. In the case of MACS2129, the cluster with fewer spectroscopic members available (11), we retrieve $\mathcal{K}=64$ percent and $\mathcal{I}=27$ percent. Still, the members sample we derive for it includes 73 per cent of correct cluster members. For those clusters for which less than 10 spectroscopic redshifts were available, we use the average value of $n$, and the probability threshold derived for the other individual clusters: $n=2$ and $\mathcal{P}_{\text {thr }}=0.5$. The reader can find examples of the application of a similar selection procedure in the works by e.g. Eisenhardt et al. (2008), Vulcani et al. (2011), and Brodwin et al. (2013).

Thorough studies of SED-fitting code performance have identified and quantified their tendency to derive overconfident $\mathcal{P}(z)$. This means that the confidence intervals derived for the $z_{\text {phot }}$ are too narrow. Given that we base our photometric cluster members identification on the $\mathcal{P}(z)$ provided by EAZY, we perform a simple check to evaluate the impact of this effect on our work. In practice, we check that the distribution of spectroscopic redshifts in the cluster is comparable with the distribution obtained combining the 
photometric redshifts $\mathcal{P}(z)$ (Sheth \& Rossi 2010). Additionally, we perform the check described by Wittman, Bhaskar \& Tobin (2016) through which we find that the overconfidence of the $\mathcal{P}(z)$ we use can be corrected broadening it by applying a convolution with a $\sigma=0.2$ Gaussian. We have checked that the impact of this effect on our work is negligible in the final selection of cluster members, given that broadening the $\mathcal{P}(z)$ leads to a different calibration of the membership determination method with smaller $P_{\mathrm{thr}}$.

\section{CLUSTER MEMBERS AND FIELD REFERENCE SAMPLES}

The main objective of our study is to compare the SF activity that takes place in the inner region of intermediate-redshift clusters with the typical observed in lower density environments (i.e. field). In this section, we describe the different galaxy samples from which we derive the results of this work. In the rest of the article the samples are frequently subdivided in three increasing redshift bins $(0.2<z$, $<0.4,0.4<z<0.6$, and $0.6<z<0.9)$. The two first bins are chosen to have equal number of clusters (11), while the last one includes only the two highest redshift ones. Furthermore, the samples are divided into three cluster-centric distance $(\mathcal{R})$ bins. The first bin $\left(\mathcal{R} / R_{200}<0.1\right)$ is the only one available across the whole redshift range. The second one $\left(0.1<\mathcal{R} / R_{200}<0.2\right)$ is visible in the two highest redshift bins. Finally, the third one $\left(0.2<\mathcal{R} / R_{200}<0.3\right)$ is covered only in the highest redshift clusters. Table 5-7 show the number counts and average properties of the various galaxy clusters subsamples. Table 8 displays the number counts and average properties of field galaxy samples.

\subsection{Samples of cluster members}

For each CLASH+HLS field, we build a general cluster members sample out of the previously described CLASH parent catalogues. We consider only sources with a $>3 \sigma$ detection in IRAC $4.5 \mu \mathrm{m}$ band to avoid spurious and extremely faint systems, and fluxes larger than the average limiting fluxes at $3 \sigma$ level (see Table 3 for the limiting fluxes at $5 \sigma$ detection level). Using the methodology described in Section 6, we select a total of 3121 cluster members distributed into the 24 clusters analysed. This number does not include the 259 galaxies for which the SED fitting is not able to derive an accurate value of mass: those sources fitted with a template of an active galaxy and sources with fewer than four photometric data points.

Fig. 3 represents the distribution with redshift of the $\mathcal{M}_{*}$ estimations derived through the SED fitting (Section 5) for the cluster members parent sample. We also represent the $\mathcal{M}_{*}$ limits given the $3 \sigma$ IRAC $4.5 \mu \mathrm{m}$ limit fluxes for each cluster (see Table 3). This conservative estimations are performed using the same set of templates described in Section 5 with solar metallicity, $\tau=1 \mathrm{Myr}$, and an age that corresponds to the age of the Universe at each redshift.

To create comparable galaxy samples at different redshifts, we focus our analysis on cluster members with $\log _{10} \mathcal{M}_{*} / M_{\odot}>10$. Our final cluster members sample contain 1518 galaxies.

We have performed a comparison between the cluster members we select using our approach and the members catalogues published by Connor et al. (2017) for all CLASH clusters. On average, $90_{-7}^{+3}$ percent of the galaxies with $\log _{10} \mathcal{M}_{*} / M_{\odot}>10$ in each of our samples have a counterpart in their general catalogues. Among them, $87_{-8}^{+9}$ per cent are also considered cluster members by Connor et al. (2017). Finally, only a $6_{-4}^{+14}$ percent of galaxies included in the cluster members catalogues of their publication are not included in our cluster members samples. Therefore, in this range of stellar masses, the differences are within our estimated levels of completeness and contamination.

\subsection{Samples of field galaxies}

In order to build a reference sample to which compare the properties of the cluster members, we make use of the outstanding data sets available on three of the CANDELS fields (Grogin et al. 2011; Koekemoer et al. 2011). In particular, we focus on both the GOODS fields (Giavalisco et al. 2004; see appendices A1 and A2) and COSMOS (Scoville et al. 2007; see appendix A3).

Using an analogous approach to that described in Sections 3-5, we create multiwavelength catalogues and derive the photometric redshifts and physical properties (e.g. $\mathcal{M}_{*}$ and $\mathcal{S F R}$ ) of the galaxies in CANDELS catalogues. Then, we apply the same spectroscopic and photometric redshift criteria to select a field sample corresponding to each cluster members sample in terms of redshift range. Then, for each field sample, we select only the galaxies with a $>3 \sigma$ detection in IRAC $4.5 \mu \mathrm{m}$ band and a IRAC $4.5 \mu \mathrm{m}$ flux larger than the $3 \sigma$ detection limit of each corresponding cluster sample. Fig. 3 represents the distribution of the field samples in the $\mathcal{M}_{*}-z$ plane.

The final field parent sample contains 7466 systems with $\log _{10} \mathcal{M}_{*} / M_{\odot}>10$. We exclude the 360 galaxies without a robust mass estimation (see previous section).

\subsection{Samples of star-forming and passive galaxies}

We divide the samples of field and cluster galaxies into star-forming and passive using the rest-frame $U-V$ versus $V-J$ colour-colour space (hereafter, $U V J$ diagram). Different works (e.g. Wuyts et al. 2007; Williams et al. 2009) have evidenced the power of the $U V J$ diagram to select pure samples of either quiescent and SFGs (e.g. Wuyts et al. 2007; Brammer et al. 2011; Whitaker et al. 2012a, 2015). In particular, we identify passive galaxies (hereafter, UVJP) following the recipes by Williams et al. (2009) for the redshift bins $0<z<0.5(U-V>0.88 \times V-J+0.69, U-V>1.3$, and $V$ $-J<1.6)$ and $0.5<z<1.0(U-V>0.88 \times V-J+0.59, U-V$ $>1.3$, and $V-J<1.6$ ). Galaxies with rest-frame $U-V$ and $V-J$ behaving otherwise are classified as star-forming (hereafter, $U V J-$ $\mathrm{SF})$. We perform Monte Carlo simulations to assess the reciprocal contamination between the two types of galaxies considering the uncertainties in the synthetic photometry. We retrieve $\leq 1$ per cent differences in the number counts of either category and sample. We find that in the clusters (field) samples, 25 per cent ( 5 per cent) of SFGs could be classified as passive given their error bars and 28 per cent (22 per cent) of passive galaxies could be classified as SFGs. We have checked that excluding the galaxies in the vicinities of the limits between the $U V J-\mathrm{P}$ and the $U V J-\mathrm{SF}$ loci do not change the results of our work significantly. This is probably due to the fact that these transition galaxies present similar properties on either side of the border.

In Fig. 4, we show the UVJ diagram for the cluster and field samples. As we can see, some galaxies detected in the FIR (i.e. presumably SFGs) are located in the region theoretically populated by passive galaxies. This contamination has been reported in the past (see e.g. Domínguez Sánchez et al. 2016) and evidences the necessity of a correction of the aforementioned selection criteria. In the final $U V J-\mathrm{SF}(U V J-\mathrm{P})$ samples, we include (exclude) both the 


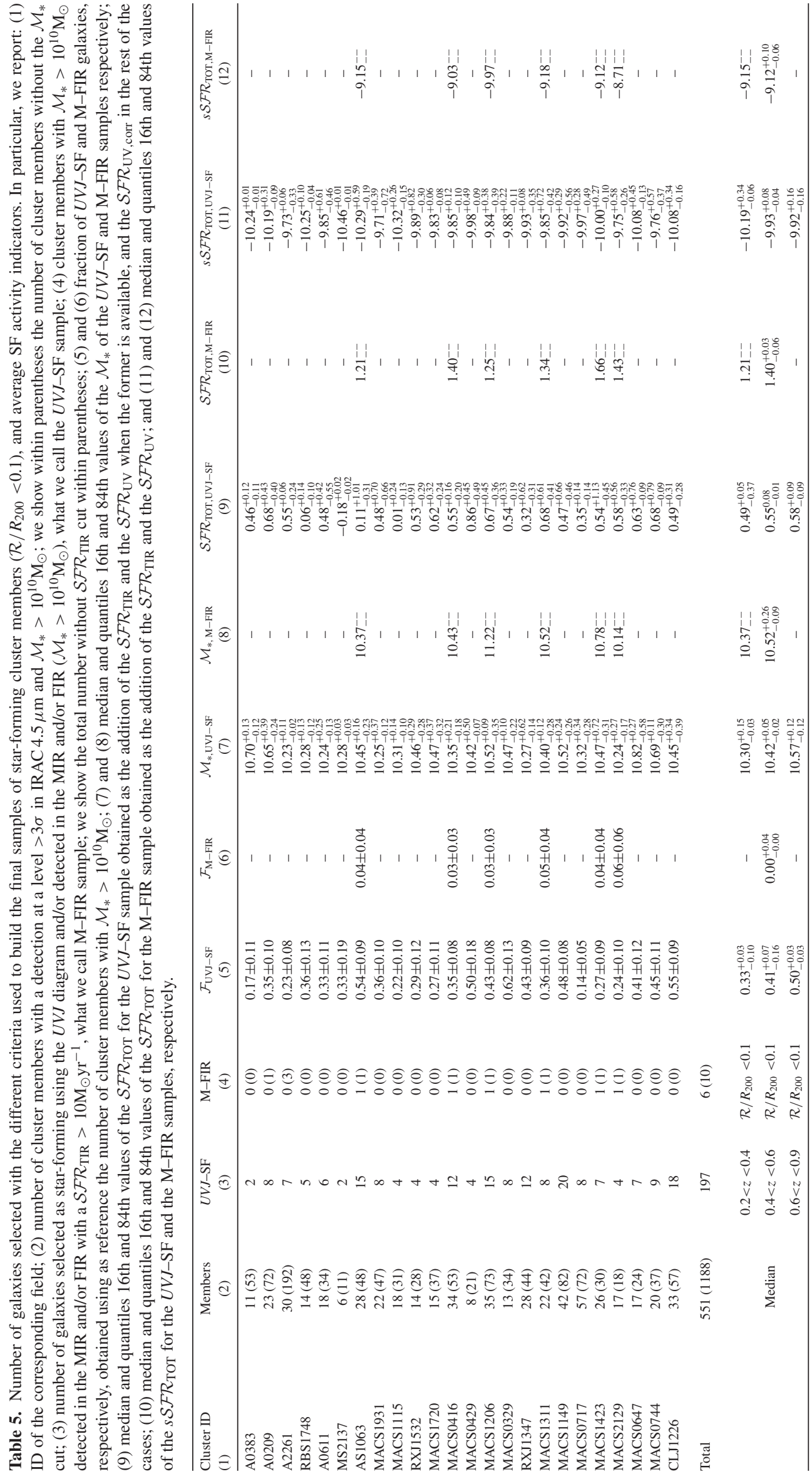




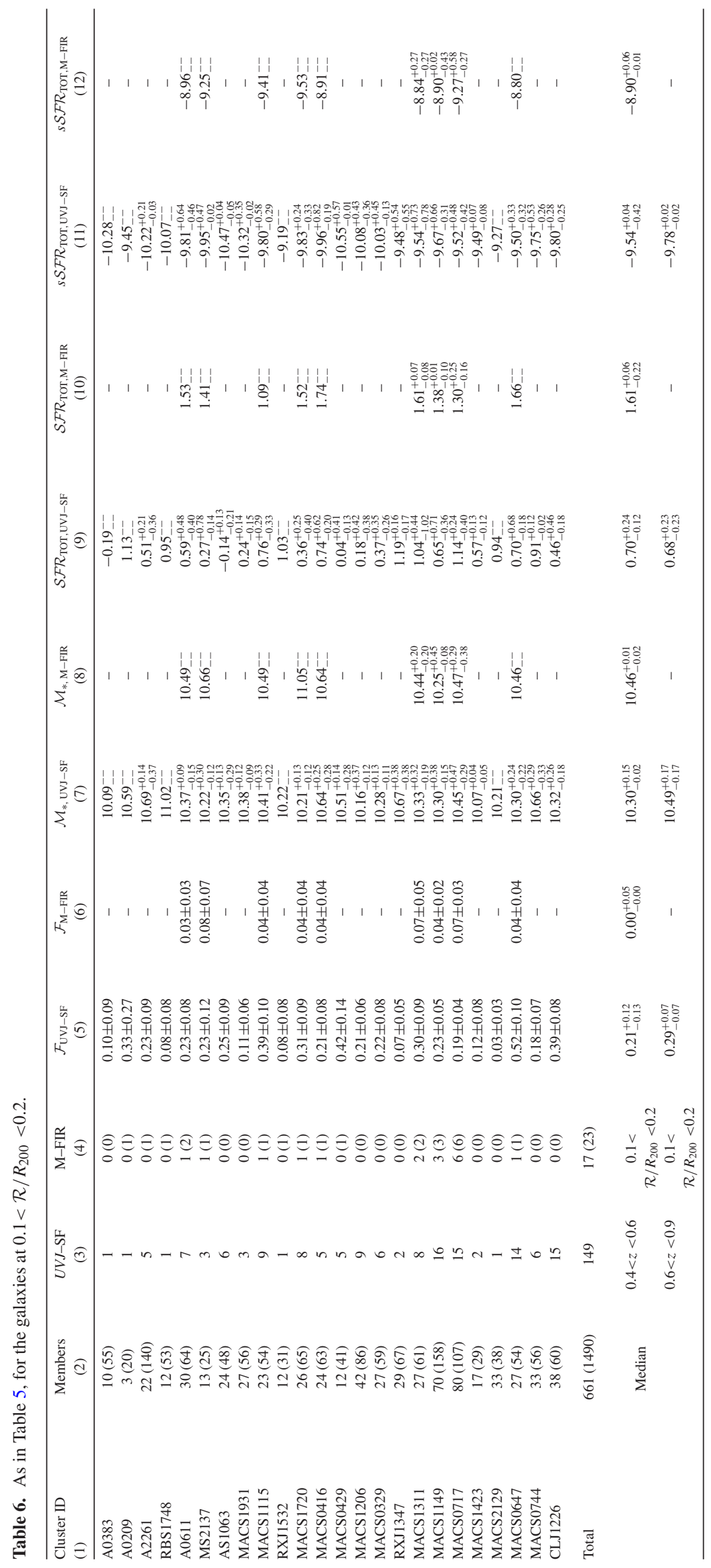




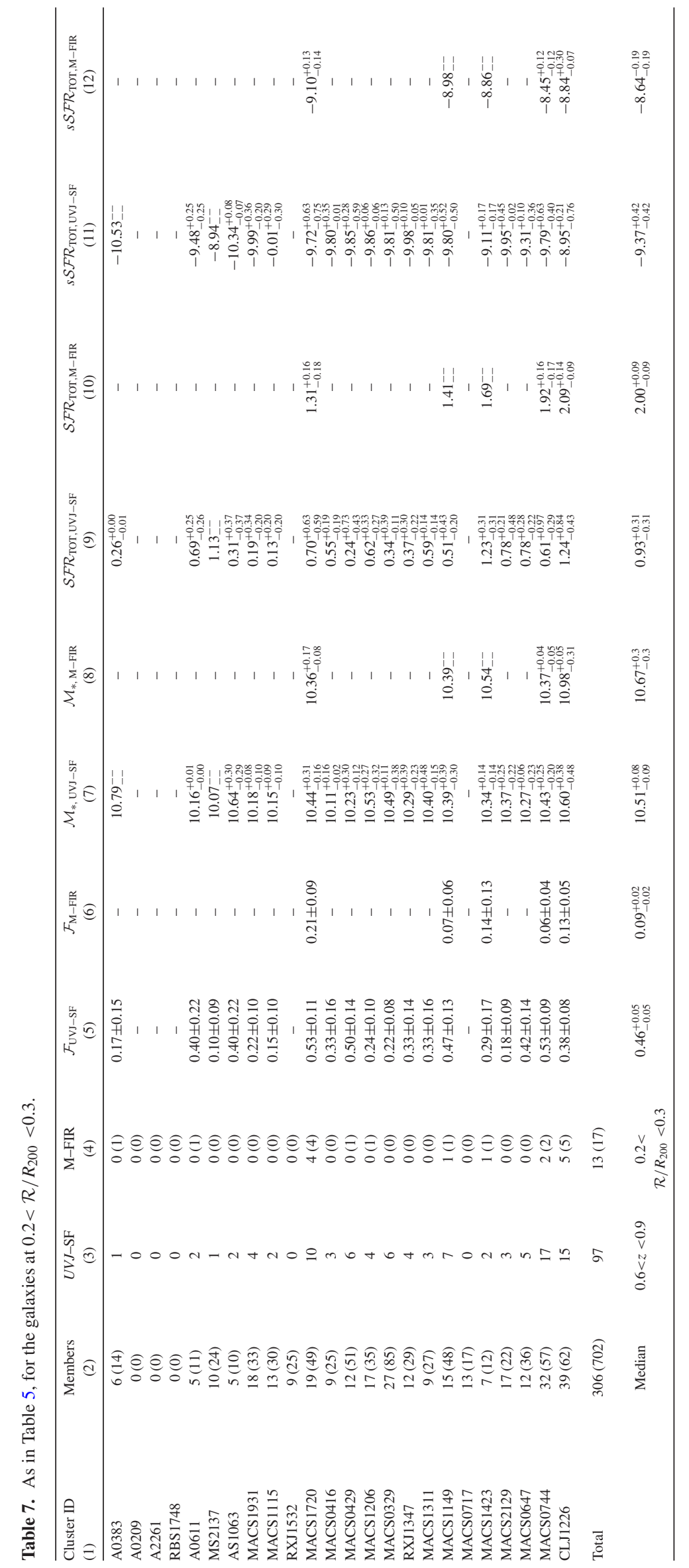




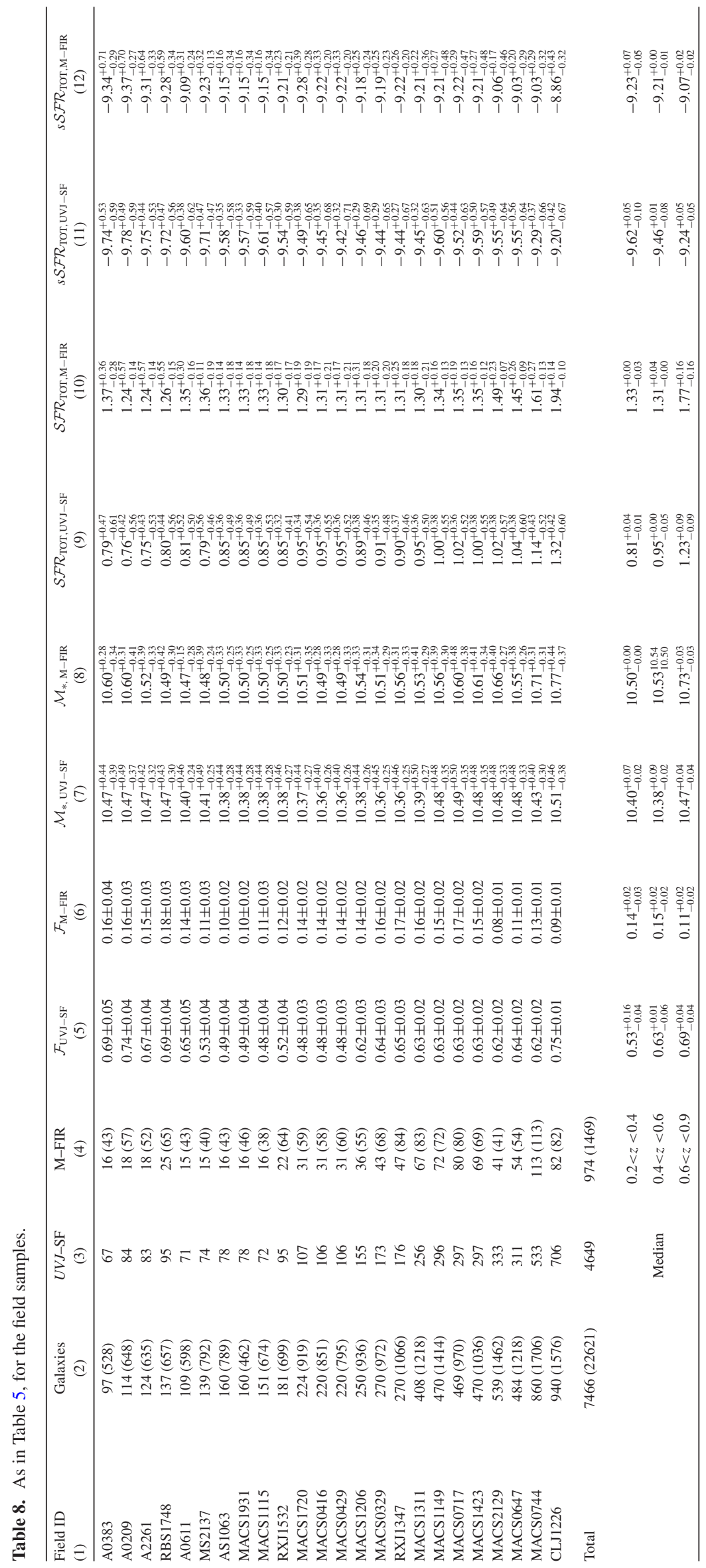




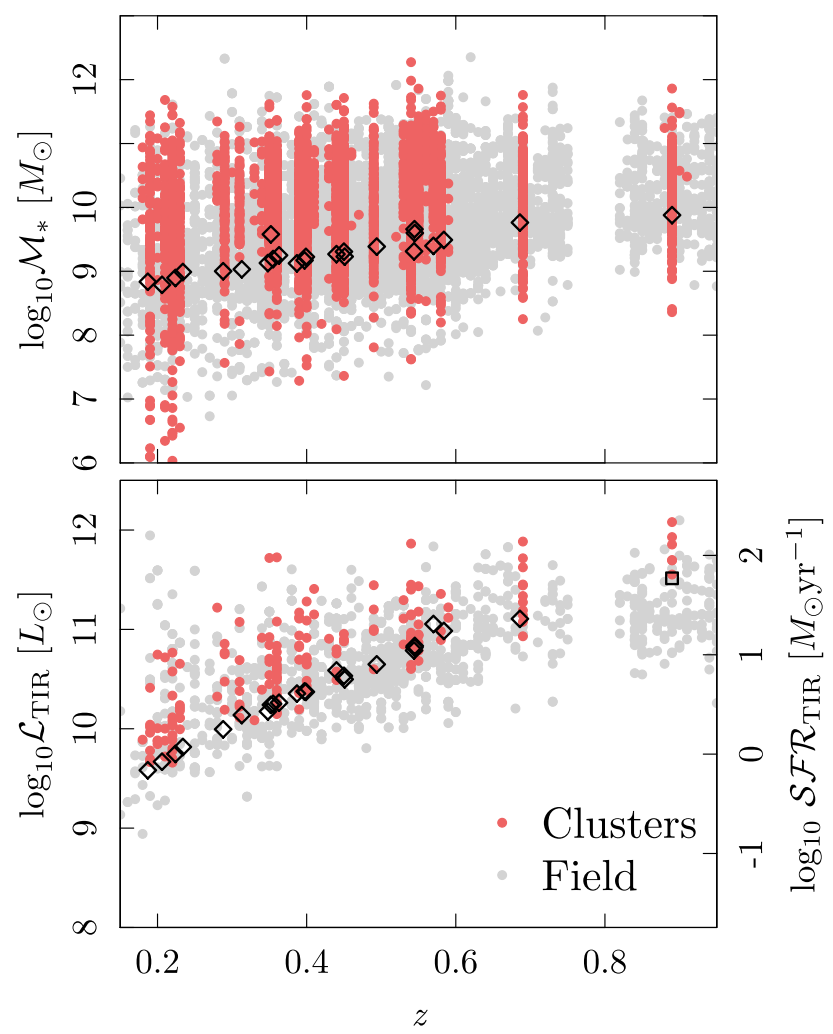

Figure 3 Top panel: distribution of $\mathcal{M}_{*}$ of the cluster members and field galaxies samples (IRAC $4.5 \mu \mathrm{m} 3 \sigma$ detection) with redshift. The mass limits obtained for each cluster as it is described in the text are marked with black diamonds. Bottom panel: variation with redshift of the total IR luminosities (left-hand axis) and $\mathcal{S F R} \mathcal{R}_{\mathrm{TIR}}$ (right-hand axis) of the parent samples of MIR/FIR-detected sources. In the case of the field, we show the sample prior to the $\mathcal{S F} \mathcal{R}_{\text {TIR }}$ cuts described in the text. Black symbols indicate the $\mathcal{L}_{\mathrm{TIR}}$ and $\mathcal{S} \mathcal{F} \mathcal{R}_{\mathrm{TIR}}$ corresponding to the limiting flux of MIPS $24 \mu \mathrm{m}$ (squares) or PACS $100 \mu \mathrm{m}$ (diamonds). For each cluster only the deepest limit is represented. In both panels, red symbols indicate cluster galaxies, and grey symbols indicate field galaxies. The number of field galaxies in this plot has been downsampled to 30 per cent of the original sample size, for visualization purposes.

galaxies located in the SFGs locus of the $U V J$ diagram and those detected in the MIR/FIR (see Section 7.4) independently of their position in the $U V J$ diagram. This correction increases (decreases) 1 percent ( 1 percent) and 2 percent ( 5 per cent) the number of star-forming (passive) galaxies in the cluster and field samples, respectively.

The $U V J-S F(U V J-\mathrm{P})$ samples built in CLASH-HLS clusters and the field include 443 (1075) and 4649 (2817) $\log _{10} \mathcal{M}_{*} / M_{\odot}>10$ galaxies, respectively.

An alternative methodology to select SFGs uses a threshold of $s \mathcal{S F R}$ under which a galaxy is considered to be passive (e.g. Kimm et al. 2009). In Fig. 5, we represent the $s \mathcal{S F \mathcal { R }}$ ТОТ $-\mathcal{M}_{*}$ diagrams for the $U V J-\mathrm{SF}$ samples in the redshift bins of Fig. 4. We can see that our $U V J-\mathrm{SF}$ selection criteria corresponds approximately to $\log _{10} s \mathcal{S} \mathcal{F} \mathcal{R}_{\mathrm{TOT}} / \mathrm{yr}^{-1} \gtrsim-10.5$.

On the left-hand half of Fig. 6, we display the distribution of the $U V J-S F$ samples selected in the clusters and the field on the $\mathcal{S} \mathcal{F} \mathcal{R}_{\text {TOT }}-\mathcal{M}_{*}$ plane. The blue shaded area illustrates the effective definition of the $U V J-\mathrm{SF}$ samples considered in the rest of the work. For comparison, we also represent the MS defined by Renzini \&

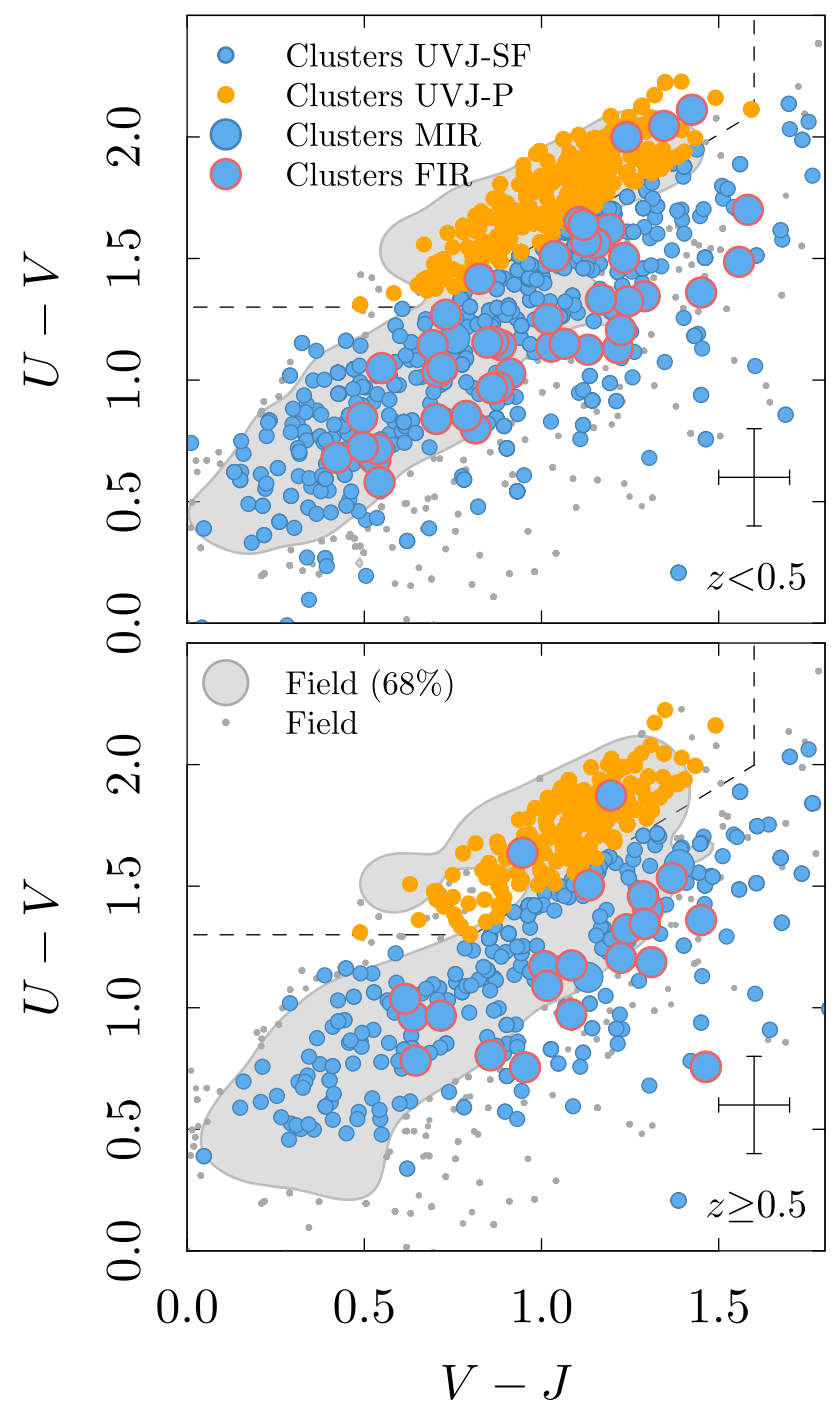

Figure $4 U V J$ diagram for the cluster members (circles) and field galaxies (grey contours and points) in two redshift bins (top panel $0<z<0.5$; and bottom panel, $0.5<z<1.0$ ). Dashed lines mark the corresponding boundaries defined by Williams et al. (2009) to distinguish between quiescent and SFGs. The circles that represent those cluster members within the locus of the passive (star-forming) galaxies are coloured in orange (blue). The cluster members detected in the FIR are highlighted with larger blue circles and a red border.

Peng (2015, black line) scaled to the median redshift of the bin, assuming and evolution with redshift of the $s \mathcal{S F R}$ of the shape (1 $+z)^{2.8 \pm 0.1}$ (Sargent et al. 2012). We notice a systematic offset of the distribution of cluster SFGs towards lower $\mathcal{S F R}$ at fixed $\mathcal{M}_{*}$ (see also Fig. 5). The quantification of this difference can be found in Section 9.3.

\subsection{Samples of MIR- and/or FIR-detected galaxies}

In order to build comparable samples of galaxies $\left(\log _{10} \mathcal{M}_{*} / M_{\odot}>10\right)$ detected in the MIR- and/or FIR (MFIR samples), we perform the following steps. First, we select galaxies with a $>3 \sigma$ detection at least in one of the MIR and/or FIR bands available (i.e. MIPS $24 \mu \mathrm{m}$, PACS 100 and $160 \mu \mathrm{m}$, and SPIRE 250, 350, and 500 $\mu \mathrm{m}$ ), and flux larger than the limiting 


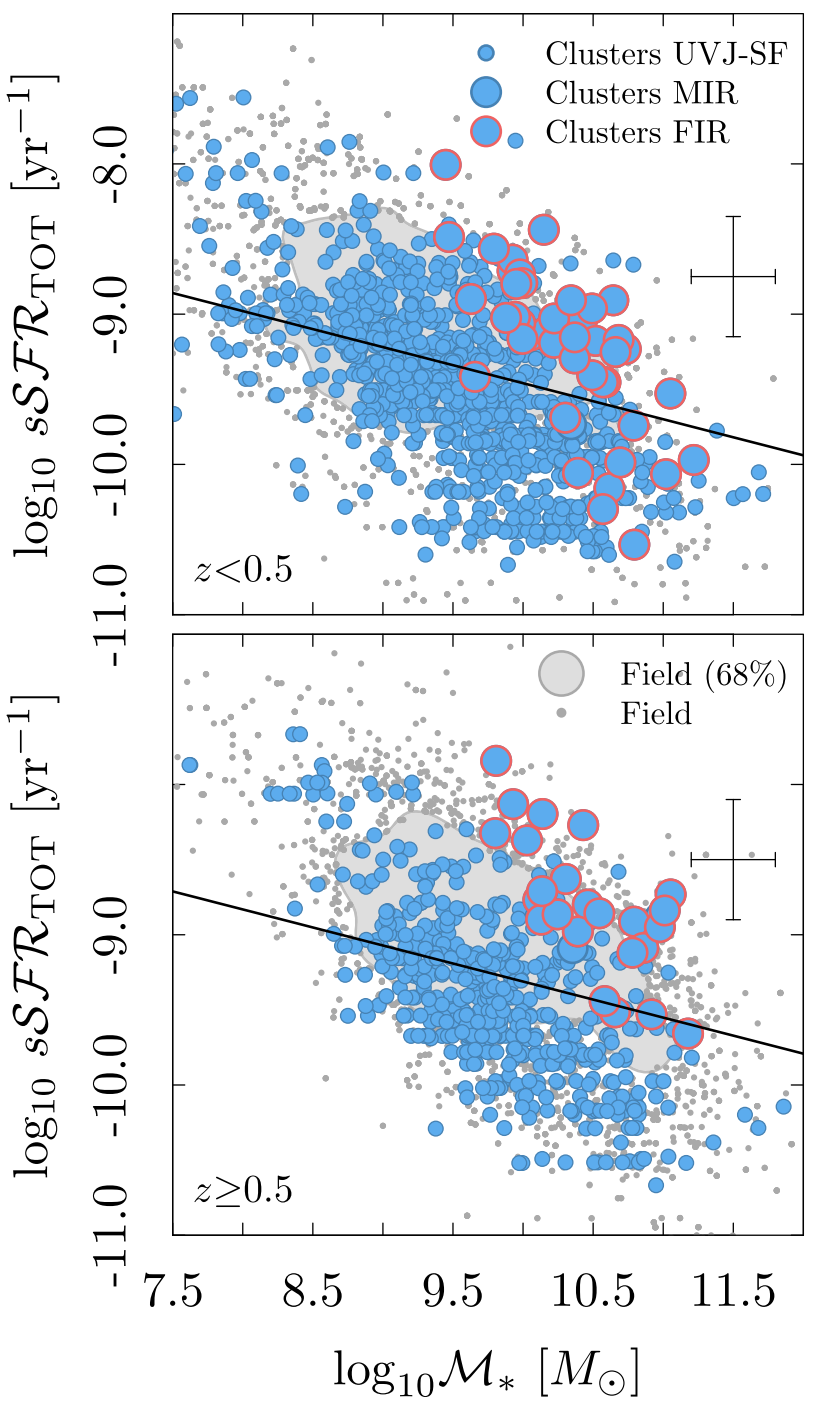

Figure $5 s \mathcal{S F R}$ Tот versus $\mathcal{M}_{*}$ for the star-forming cluster members (blue points) and field galaxies (grey contours and points) in the two redshift bins in Fig. 4. The cluster members detected in the FIR are highlighted with larger blue circles and a red border. The black lines represent the MS by Renzini \& Peng (2015) scaled to the median redshift of the corresponding bin considering an evolution with redshift of the $s \mathcal{S F R}$ of the shape $(1+$ $z)^{2.8 \pm 0.1}$ (Sargent et al. 2012).

fluxes at $3 \sigma$ level in the clusters (see Table 3 for the limiting fluxes at $5 \sigma$ detection level). These galaxies are represented in the bottom panel of Fig. 3. Then, we select only the 50 (1496) clusters (field) galaxies for which the estimated $\mathcal{S} \mathcal{F} \mathcal{R}_{\mathrm{TIR}}$ is larger than the (conservative) $\mathcal{S} \mathcal{F} \mathcal{R}_{\mathrm{TIR}}$ limits obtained for each cluster (black symbols in the bottom panel of Fig. 3). Fig. 7 shows the thumbnails of the cluster members detected in the MIR and/or FIR. Finally, we consider galaxies with $\mathcal{S F R} \mathcal{R}_{\mathrm{TIR}}>10 M_{\odot} \mathrm{yr}^{-1}$ to obtain a comparable set of samples of galaxies throughout the whole redshift range. This value is larger than the $\mathcal{S F} \mathcal{R}_{\mathrm{TIR}}$ limits of our sample, except for the four furthest clusters. Our final $M-F I R$ samples include 36 cluster members and 974 field galaxies. On the right-hand half of Fig. 6, we display the distribution of these samples on the $\mathcal{S F} \mathcal{R}_{\mathrm{TIR}}-\mathcal{M}_{*}$ plane. The red shaded area marks the $\mathcal{M}_{*}$ and $\mathcal{S} \mathcal{F} \mathcal{R}_{\mathrm{TIR}}$ cuts performed to define the samples.
It is worth mentioning that we perform a visual inspection of each cluster member selected as a M-FIR emitter. We exclude spurious MIPS $24 \mu \mathrm{m}$ sources without a counterpart in longer wavelengths (e.g. sources on Airy ring features), galaxies in the borders of the images that are selected as counterparts of MIR/FIR sources with coordinates outside the area covered by CLASH catalogues, or galaxies suffering from overdeblending in the CLASH catalogues.

Interestingly, we find eight BCGs detected in the MIR/FIR out of 24 clusters, which corresponds to 33 per cent of our sample. This percentage is consistent with the results of the study conducted by Rawle et al. (2012a) using HLS data on a sample of 68 massive galaxy clusters spread out in the redshift range between $0.08<z$ $<1.00$. Their sample includes only $12 \mathrm{CLASH}+\mathrm{HLS}$ clusters. As expected, among the BCGs of these 12 systems, we detect traces of obscured SF in the same two, namely A0383 and MACS1423. We exclude BCGs from our samples given their unique SFH and in order to focus our results on the SF activity of the general cluster galaxy population.

The fraction of AGNs among IR-bright cluster members has been observed to increase rapidly from 3 per cent up to 65 per cent for galaxies with increasing $\mathcal{L}_{\text {TIR }}$ values varying from $10^{11} L_{\odot}$ to $>10^{11.6} L_{\odot}$ in clusters within the redshift range $0.15<z<0.30$ (Haines et al. 2013). Given the SED-fitting methodology explained and sample selection, we exclude from our analysis the galaxies whose photometry was fitted to AGN templates.

The so-called luminous and ultraluminous infrared galaxies (LIRGs and ULIRGs, respectively) display $\mathcal{L}_{\mathrm{TIR}}$ in the range of $10^{11} L_{\odot}<\mathcal{L}_{\mathrm{TIR}}<10^{12} L_{\odot}$ and $\mathcal{L}_{\mathrm{TIR}}>10^{12} L_{\odot}$, respectively, which correspond to $\mathcal{S F} \mathcal{R}_{\mathrm{TIR}}$ from tens to thousands of $M_{\odot} \mathrm{yr}^{-1}$. Our MFIR sample of cluster members includes 25 LIRGs and 1 ULIRGs (within CLJ1226, the highest redshift cluster) and our M-FIR sample of field galaxies includes 639 LIRGs, and 10 ULIRGs. These numbers correspond to comparable percentages of LIRGs and ULIRGs within the M-FIR samples in clusters and field.

\section{STELLAR MASS DISTRIBUTIONS}

As a step prior to the evaluation of the SF within cluster cores and how it compares to the SF in the field, we explore the stellar mass function (SMF) of the samples presented in the previous section. The SMF is a fundamental observable for the study of the evolution of galaxy populations. Furthermore, overlooking hypothetical differences in the SMF of field and cluster samples can lead to a misinterpretation of the physics behind the level of SF quantified in the following sections.

In the top panels of Fig. 8, we display the SMF for clusters and field galaxies $\left(\log _{10} \mathcal{M}_{*} / M_{\odot}>10\right)$ divided into bins of redshift. We include only galaxies at $\mathcal{R}<0.1 R_{200}$, i.e. the $\mathcal{R}$ range homogeneously covered along the whole redshift range. We exclude the BCGs in our analysis. We correct for different cluster richnesses by randomly resampling the galaxy population of each cluster using the average sample size of each redshift bin. Then, to render the field and cluster samples statistically comparable, we resample each field drawing randomly the number of galaxies in the corresponding cluster sample. The uncertainties are estimated from the combination of 500 bootstraps. Then, we model the data by fitting a Schechter function (Schechter 1976) to the SMF. The form of the function is

$\Phi\left(\mathcal{M}_{*}\right) \mathrm{d} \mathcal{M}_{*}=\Phi^{*}\left(\frac{\mathcal{M}_{*}}{\mathcal{M}^{*}}\right)^{\alpha} \mathrm{e}^{-\frac{\mathcal{M}_{*}^{*}}{\mathcal{M}^{*}}} \frac{\mathrm{d} \mathcal{M}_{*}}{\mathcal{M}^{*}}$, 

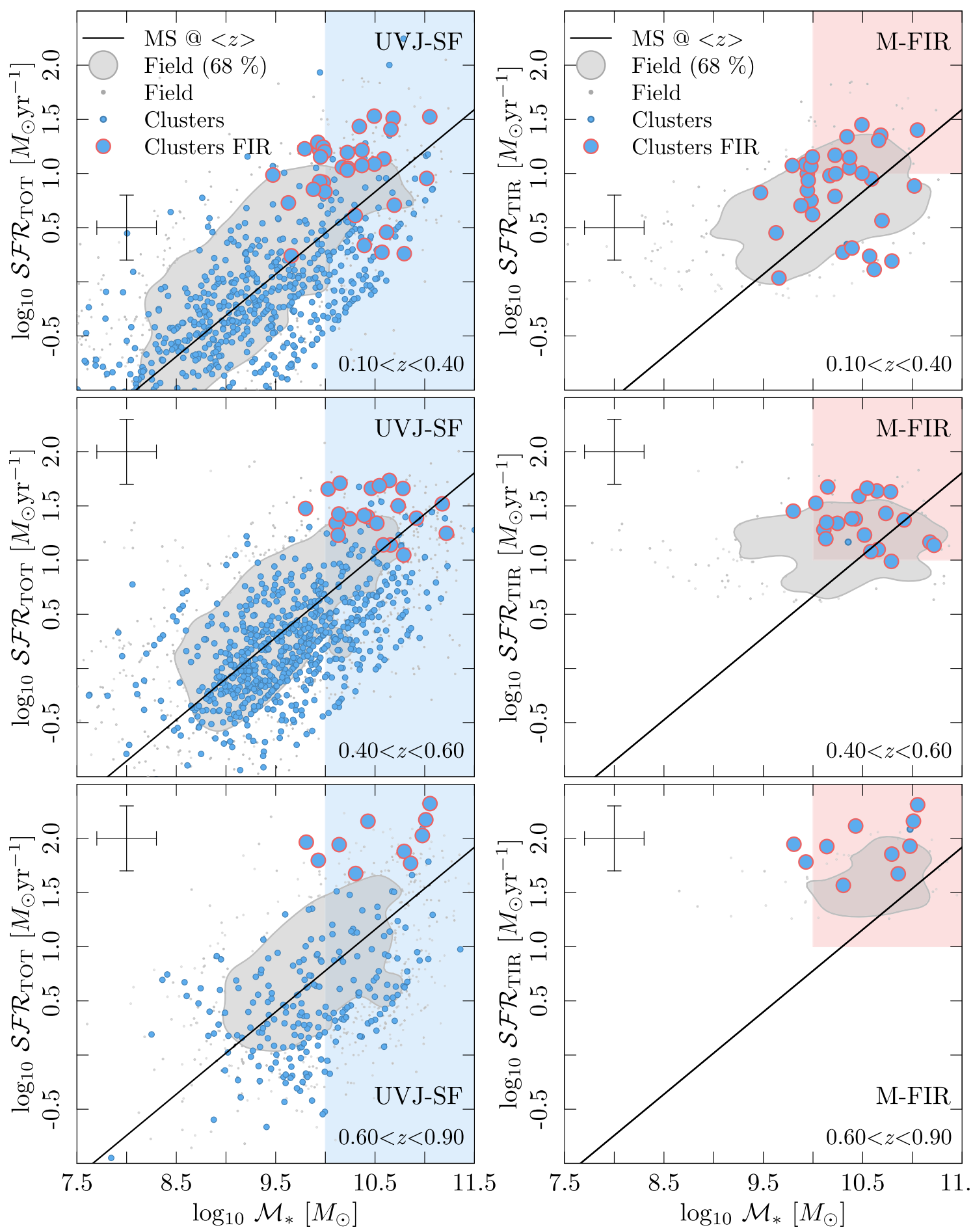

Figure $6 \mathcal{S F} \mathcal{R}_{\text {TOT }}$ versus $\mathcal{M}_{*}$ relation for the star-forming cluster members in our study split up in three increasing $z$ bins (top, middle, and bottom panels). On the (left-) right-hand panels, we include the (UVJ-SF) M-FIR galaxies across the whole mass range. The $\mathcal{S F R}$ TOT refers to the $\mathcal{S F R}$ TIR $+\mathcal{S F \mathcal { R }}$ UV for those galaxies MIR/FIR-detected, and $\mathcal{S F} \mathcal{R}_{\mathrm{UV}}$,corr otherwise. Blue points always represent the distribution of clusters members in both cases. Those galaxies detected in the FIR (i.e. Herschel) are shown with larger blue points highlighted with red borders. Grey contours represent the distribution (68 confidence levels) of field galaxies. We also display the MS by Renzini \& Peng (2015, black lines) scaled to the median redshift of the corresponding subsample of cluster members considering a trend of $s \mathcal{S F R}$ with redshift $\propto(1+z)^{2.8} \pm 0.1$ (Sargent et al. 2012). The shaded areas represent the selection criteria used to build the final samples of $U V J-S F$ and MIR-FIR galaxies (i.e. they represent the cut in $\mathcal{M}_{*}$, and $\mathcal{S F} \mathcal{R}_{\mathrm{TIR}}$ ).

with $\mathcal{M}^{*}$ being the characteristic mass, $\alpha$ the low-mass slope, and $\Phi *$ the normalization. The normalization is evaluated by requiring that the integral of the Schechter function over the stellar mass range considered equals the fraction of galaxies in the sample fitted with respect the total sample. In Table 9, we report the best-fitting parameters. The function provides overall reasonable fits, although we report a quite large scatter of the data points for some of the samples. This is probably due to the limited number counts we 


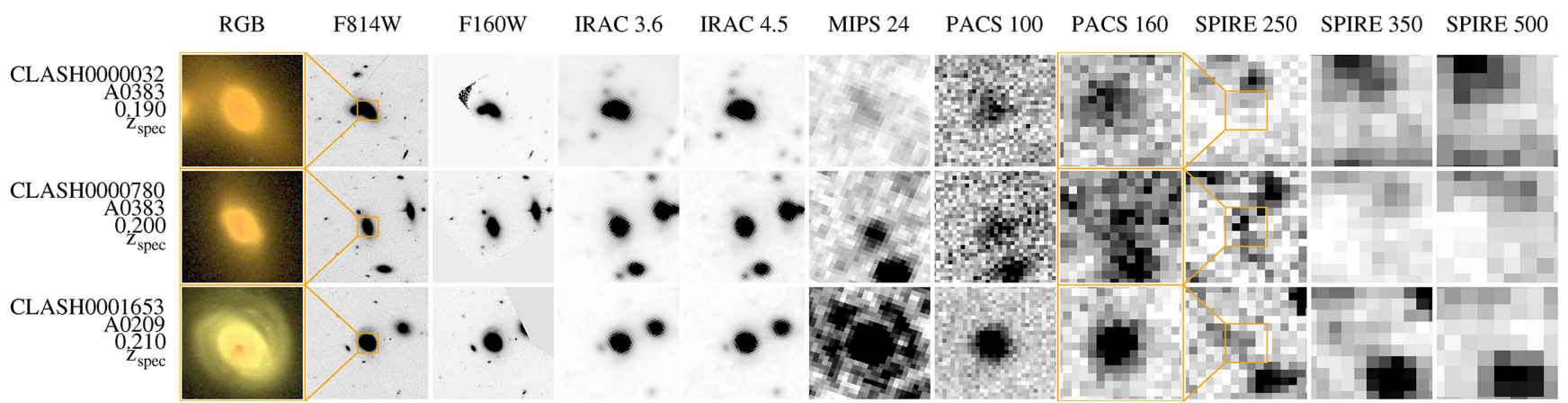

Figure 7 Thumbnails of three cluster members from the M-FIR sample ordered by increasing redshift. From left to right, we display an RGB image (5 arcsec $\times 5$ arcsec) created using HST/ACS/F814W, F606W, and F435W, following the methodology by Lupton et al. (2004), 30 arcsec $\times 30$ arcsec postage stamps in the HST/ACS/F814W and HST/WFC3/F160W bands followed by Spitzer/IRAC and MIPS, and Herschel/PACS bands, and 90 arcsec $\times 90$ arcsec postage stamps in the Herschel/SPIRE bands, all ordered by increasing wavelength. When there is a difference in the sizes of two adjacent frames, we mark with an orange square the size of the smallest on the largest. On the left-hand side, we show the ID of the object, the name of the cluster, the redshift, and either if it is photometric or spectroscopic. The thumbnails of the rest of the sample can be found as online material.
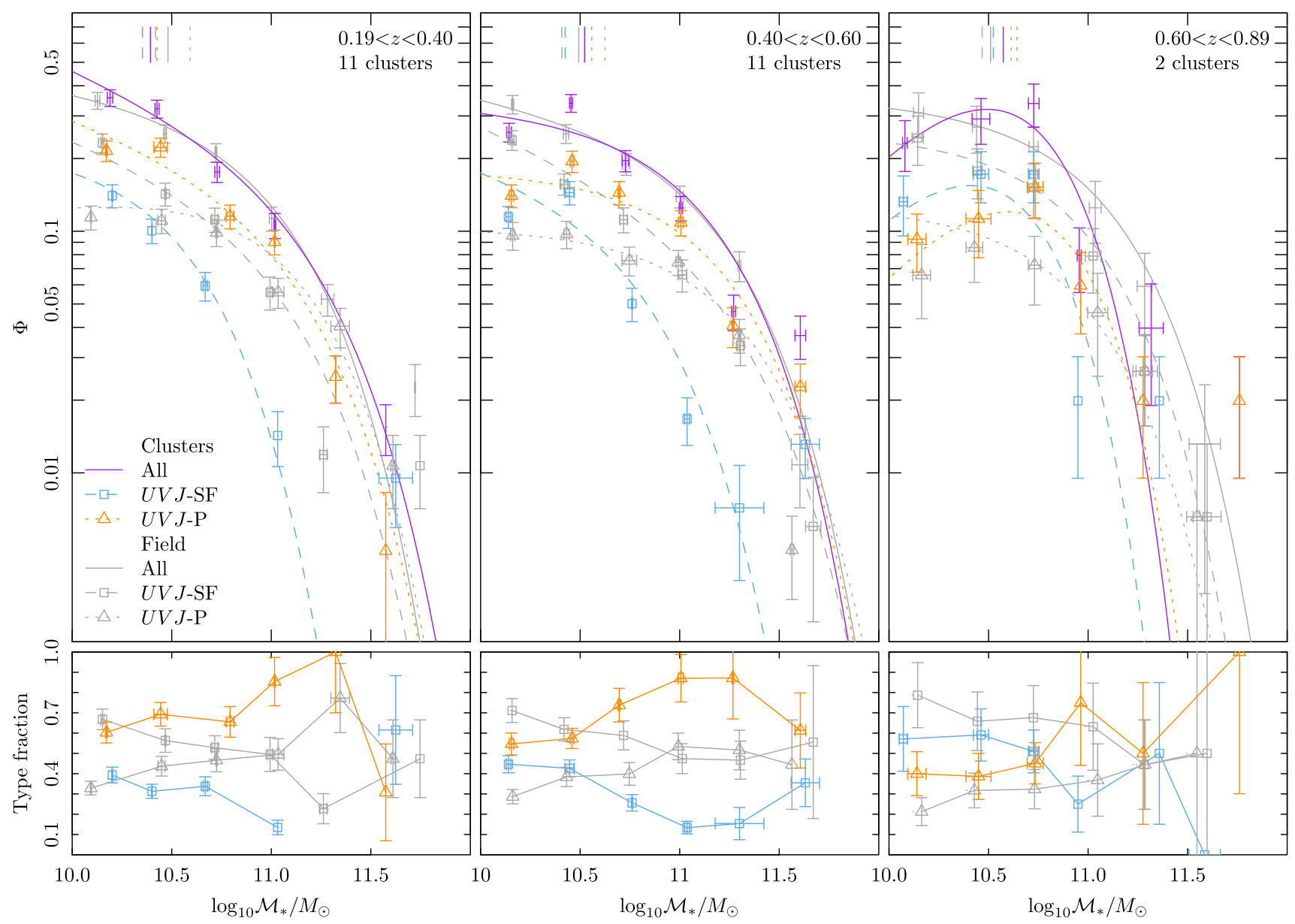

Figure 8 Top panels: stellar mass distribution within $\mathcal{R} / R_{200}<0.1$ for cluster and field galaxies $\left(\log _{10} \mathcal{M}_{*} / M_{\odot}>10\right)$ divided in bins of redshift. On the upper part of each panel, we mark the median stellar mass of every sample. Bottom panels: relative fraction of $U V J-\mathrm{P}$ and $U V J-\mathrm{SF}$ galaxies as a function of stellar mass.

work with. In the bottom panels of Fig. 8, we display the fraction of $U V J-\mathrm{P}$ and $U V J-\mathrm{SF}$ galaxies in each stellar mass bin. The plots are not perfectly symmetric because we do not fix the median value of each mass bin. We do not represent the stellar mass distribution of the M-FIR sample because its size is not statistically significant for this analysis. The median value of stellar mass corresponding to each sample is marked in the upper panels of the same figure (see also Table 9).

We compare the best-fitting Schechter parameters with those published recently by van der Burg et al. (2018) for cluster and field 
Table 9. We report: median $\log _{10} \mathcal{M}_{*}$ (and $1 \sigma$ intervals), best-fitting Schechter parameters (and $1 \sigma$ intervals) and reduced $\chi^{2}$ for the different samples.

\begin{tabular}{|c|c|c|c|c|c|c|c|}
\hline & & Sample & $<\log _{10} \mathcal{M}_{*} / M_{\odot}>$ & $\log _{10} \mathcal{M}^{*} / M_{\odot}$ & $\alpha$ & $\Phi *$ & $\chi^{2}$ \\
\hline & Field & $\begin{array}{c}\text { All galaxies } \\
U V J-\mathrm{SF} \\
U V J-\mathrm{P}\end{array}$ & $\begin{array}{c}10.48_{-0.38}^{+0.48} \\
10.42_{-0.31}^{+0.47} \\
10.59_{-0.49}^{+0.47}\end{array}$ & $\begin{array}{l}11.05_{-0.12}^{+0.12} \\
11.11_{-0.05}^{+0.05} \\
11.09_{-0.18}^{+0.18}\end{array}$ & $\begin{array}{l}-1.1_{-0.1}^{+0.1} \\
-1.3_{-0.5}^{+0.5} \\
-0.9_{-0.2}^{+0.2}\end{array}$ & $\begin{array}{c}0.13_{-0.04}^{+0.04} \\
0.05_{-0.00}^{+0.00} \\
0.07_{-0.02}^{+0.02}\end{array}$ & $\begin{array}{l}5.69 \\
5.61 \\
5.41\end{array}$ \\
\hline $0.40<z<0.60$ & Field & $\begin{array}{c}\text { All galaxies } \\
U V J-\mathrm{SF} \\
U V J-\mathrm{P}\end{array}$ & $\begin{array}{c}10.49_{-0.35}^{+0.52} \\
10.41_{-0.28}^{+0.53} \\
10.63_{-0.41}^{+0.49}\end{array}$ & $\begin{array}{l}11.22_{-0.02}^{+0.02} \\
11.30_{-0.09}^{+0.09} \\
11.32_{-0.13}^{+0.13}\end{array}$ & $\begin{array}{l}-1.2_{-0.1}^{+0.1} \\
-1.4_{-0.1}^{+0.1} \\
-1.0_{-0.5}^{+0.5}\end{array}$ & $\begin{array}{l}0.11_{-0.01}^{+0.01} \\
0.04_{-0.01}^{+0.01} \\
0.05_{-0.01}^{+0.01}\end{array}$ & $\begin{array}{l}6.35 \\
1.49 \\
6.07\end{array}$ \\
\hline & Field & $\begin{array}{c}\text { All galaxies } \\
U V J-\mathrm{SF} \\
U V J-\mathrm{P}\end{array}$ & $\begin{array}{c}10.51_{-0.35}^{+0.46} \\
10.47_{-0.34}^{+0.44} \\
10.64_{-0.37}^{+0.50}\end{array}$ & $\begin{array}{l}11.11_{-0.05}^{+0.05} \\
11.00_{-0.50}^{+0.50} \\
11.00_{-0.29}^{+0.29}\end{array}$ & $\begin{array}{l}-1.0_{-0.1}^{+0.1} \\
-1.0_{-0.5}^{+0.5} \\
-1.0_{-0.2}^{+0.2}\end{array}$ & $\begin{array}{c}0.14_{-0.02}^{+0.02} \\
0.10_{-0.01}^{+0.01} \\
0.05_{-0.03}^{+0.03}\end{array}$ & $\begin{array}{l}1.22 \\
7.86 \\
0.58\end{array}$ \\
\hline
\end{tabular}

galaxies at $0.5<z<0.7$. We focus our comparison on their inner $\mathcal{R}$ bin $\left(\mathcal{R} / R_{200} \lesssim 0.4\right)$. Their $\log _{10} \mathcal{M}^{*} / M_{\odot}$ are $11.01_{-0.02}^{+0.02}, 11.01_{-0.02}^{+0.01}$, and $10.70_{-0.04}^{+0.04}$ for the whole population, the quiescent, and the star-forming samples of the clusters, respectively, and $11.18_{-0.02}^{+0.02}$, $11.06_{-0.02}^{+0.02}$, and $10.89_{-0.05}^{+0.05}$ for the same subsamples in the field. We assume a 0.2 dex conversion from Chabrier (Chabrier 2003) to Salpeter IMF (Conroy, Gunn \& White 2009). Our results for the clusters and field between $0.4<z<0.6$ are compatible with theirs except in the case of the cluster $U V J-\mathrm{P}$ sample, for which we derive $\log _{10} \mathcal{M}^{*} / M_{\odot}=11.22_{-0.06}^{+0.06}$, and the field $U V J-S F$ and $U V J-$ $\mathrm{P}$ populations, for which we derive larger values: $\log _{10} \mathcal{M}^{*} / M_{\odot}=$ $11.30_{-0.09}^{+0.09}$, and $11.32_{-0.13}^{+0.13}$, respectively. Regarding $\alpha$, they retrieve $-0.91_{-0.02}^{+0.02},-0.83_{-0.02}^{+0.03}$, and $-1.02_{-0.06}^{+0.06}$ for the whole population, the quiescent, and the star-forming samples of the clusters, and $-1.20_{-0.02}^{+0.02},-0.55_{-0.03}^{+0.03}$, and $-1.33_{-0.03}^{+0.03}$ for the field. In this case, our results are compatible with theirs within the error bars.

In the first two redshift bins, there are no large differences between the SMF of the whole population of galaxies in the field and the clusters, with values of the slope and the knee of the Schechter function within the $1 \sigma$ errors (see Table 9). This result has been found in previous works at intermediate and high redshifts (e.g. Vulcani et al. 2012, 2013; van der Burg et al. 2013; Nantais et al. 2016). On the contrary, the highest redshift bin displays large differences between the cluster and the field best-fitting Schechter functions. We claim these differences are mainly due to a poor sampling of the cluster SMF. In fact, data points in the stellar mass range including 80 percent of the stellar mass of both cluster and field samples are compatible within the error bars.

We report hints of a different behaviour of the SMFs of field and clusters and their evolution with $z$ when we split the galaxy populations in $U V J-\mathrm{SF}$ and $U V J-\mathrm{P}$. At the lowest redshift, the $U V J-$ P SMF appears to present a steeper $\alpha$ than the field, which is not obvious in the second redshift bin. This makes the UVJ-P SMF present a shape apparently more similar to the field $U V J-\mathrm{SF}$ stellar mass distributions (excluding normalization differences). Balogh et al. (2001) also find that while in the field environment the SMF of SFGs has much steeper faint-end slope than that for passive galaxies, in the clusters, the passive galaxies have also a steep faint end. Annunziatella et al. (2014) find that for the $z=0.44$ (our second redshift bin) cluster MACS1206 (also included in our sample), the SMF of SFGs is significantly steeper than the SMF of passive galaxies at the faint end. This is in agreement with our bestfitting SMFs in the intermediate-redshift bin. Furthermore, they find a smaller slopes SMF for passive cluster galaxies in the inner core of clusters $\left(\mathcal{R} / R_{200} \lesssim 0.25\right)$, than in the outskirts.

However, these differences are not significant in most cases. The best-fitting values of $\alpha$ and $\log _{10} \mathcal{M}^{*}$ for the $U V J-S F$ and $U V J-\mathrm{P}$ samples in the clusters and field are overall compatible within the error bars. The only significant difference appears in the value of the $\log _{10} \mathcal{M}^{*} / M_{\odot}$ for the $U V J-S F$ samples in the lowest redshift bin: $10.55_{-0.06}^{+0.06}$ and $11.11_{-0.05}^{+0.05}$ for the clusters and the field, respectively. Other works have also reported the lack of significant differences between the SMF of SFGs and passive galaxies in different environments (i.e. Vulcani et al. 2013). The $U V J-\mathrm{SF}$ and $U V J-\mathrm{P}$ SMF evolution with redshift is also mild in terms of the best-fitting Schechter parameters $\alpha$ and $\log _{10} \mathcal{M}^{*}$, and considering our resolution.

In the first two redshift bins, we find that the galaxy population in massive clusters is clearly dominated by quiescent galaxies all the way down to $\mathcal{M}_{*}=10^{10} M_{\odot}$, which is in agreement with (e.g.) van der Burg et al. (2018). The largest mass bins are dominated by stochasticity given the small number of galaxies included. Peng et al. (2010) predict that the SMFs of passive and SFGs should cross (crossing mass) at $\log _{10} \mathcal{M}_{*} / M_{\odot} \approx 10.4$ and 9.6 for central ('field') and satellites, respectively, at low redshift. In our work, the crossing mass for the cluster SMFs shows up at $\log _{10} \mathcal{M}_{*} / M_{\odot} \approx 10$ in the second redshift bin. In the third redshift bin, the contribution of $U V J-\mathrm{SF}$ and $U V J-\mathrm{P}$ samples to the whole population of clusters is 
$\approx 50$ per cent, with type fractions comparable within the error bars. This is comparable with the $U V J-\mathrm{P}$ and $U V J-\mathrm{SF}$ type fractions derived by Nantais et al. (2016) for $z \sim 1.5$. Regarding the field, lower mass bins $\left(\log _{10} \mathcal{M}_{*} / M_{\odot}<10.6,10.9\right.$, and 10.9 for the first, second, and third redshift bins, respectively) are dominated by SFGs, whereas the contribution of $U V J-\mathrm{P}$ and $U V J-\mathrm{SF}$ galaxies tend to converge and even to be inverse towards higher mass bins. Other previous studies (e.g. Quadri et al. 2012, Nantais et al. 2016, Papovich et al. 2018) have claimed a rapid increase in the number density of low- and intermediate-mass $\left(\log _{10} \mathcal{M}_{*} / M_{\odot}<10\right.$ 10.6) quiescent galaxies in denser environments since $z \approx 1.5$. Moutard et al. (2018) and Mortlock et al. (2015) also find evidence for a higher number density of quiescent low-mass galaxies in denser environments in our same redshift range. However, our $\mathcal{M}_{*}$ completeness levels hamper the analysis of a possible evolution of the distribution of stellar mass at such low values.

It is worth noting that numerous works (e.g. Annunziatella et al. 2016) find that passive cluster galaxies are better fitted by a double Schechter function, revealing the existence of two subpopulations of red cluster members thought to have followed distinct evolutionary paths. On the one hand, a population of high-mass galaxies thought to be quenched by processes scaling with stellar mass, and on the other hand, a population of low-mass galaxies quenched by environmental processes (Peng et al. 2010). These composite SMF of red passive galaxies have also been observed in the field in works such as, e.g. Drory et al. (2009) and Baldry et al. (2012). However, the evidence for these double Schechter functions (i.e. an upturn at low stellar masses) is only visible at $\log _{10} \mathcal{M}_{*} / M_{\odot} \lesssim 10$ (Drory et al. 2009), below the mass limit of our work.

\section{QUANTIFICATION OF STAR FORMATION PROCESSES WITHIN CLUSTER CORES}

In this section, we present a quantification of the SF activity hosted by cluster members and field galaxies with $\log _{10} \mathcal{M}_{*} / M_{\odot}>10$, as traced by the UV and the MIR/FIR.

\subsection{Star-forming galaxy fraction}

Fig. 9 (left-hand panel) shows the fraction $(\mathcal{F})$ of $U V J-S F$ and MFIR galaxies $\left(\mathcal{F}_{\mathrm{UVJ}-\mathrm{SF}}\right.$ and $\mathcal{F}_{\mathrm{M}-\text { FIR }}$, respectively; Section 7.4$)$ in the clusters $\left(\mathcal{R} / R_{200}<0.1\right)$ and field. Error bars are obtained using the margin of error of a percentage ${ }^{7}$ assuming a standard normal distribution. On the right-hand panel, we show the median $\mathcal{F}$ and quantiles 16th and 84th (in the shape of error bars) in the same redshift bins of Fig. 6. We also include with larger symbols the fractions obtained at $0.1<\mathcal{R} / R_{200}<0.2$ and $0.2<\mathcal{R} / R_{200}<0.3$, at the corresponding redshift bins. In all cases, the median and quantiles are obtained using the bootstrap methodology.

To quantify the trends of $\mathcal{F}$ with redshift, we fit to the data points (fraction for each individual cluster within $\mathcal{R} / R_{200}<0.1$ ) a function with the shape $\alpha(1+z)^{\beta}$, where $\alpha$ corresponds to the value of $\mathcal{F}$ at $z=0$, and $\beta$ describes its evolution with redshift (with larger values of $\beta$ meaning a steeper trend). This methodology is also applied by, for example Haines et al. (2013) and Alberts et al. (2014). The

\footnotetext{
${ }^{7}$ The confidence interval of a point sample estimate of the population proportion at $1 \sigma$ can be derived considering a standard normal distribution with the expression $\sqrt{p(1-p) / n}$, where $n$ is the size of the sample and $p$ is the proportion. Both of them must satisfy the condition that $n p \geq 5$ and $n(1-p) \geq 5$.
}

corresponding curves and $1 \sigma$ confidence intervals (generated using Monte Carlo simulations) are overplotted in Fig. 9 with a coloured line and a shaded area around it, respectively. Table 10 shows the $\alpha$ and $\beta$ values of the best fit. In the case of the M-FIR samples, we fit only the clusters with an $\mathcal{S F R}$ TIR limit below $10 M_{\odot} \mathrm{yr}^{-1}(z$ $<0.570)$ to derive the redshift trend.

The first information we can derive from Fig. 9 is that, as expected, the $\mathcal{F}$ within clusters is much smaller than in the field for both $U V J-\mathrm{SF}$ and M-FIR samples. On average, $\mathcal{F}_{\mathrm{UVJ}-\mathrm{SF}}$ in clusters seems to be approximately half the value in the field. The $\mathcal{F}_{\text {M-FIR }}$ in clusters drop down to values not significantly different to zero. Assuming the same fraction of M-FIR galaxies among the SFGs in clusters and field, the expected average $\mathcal{F}_{\text {M-FIR }}$ for the former would be $\sim 5$ per cent, which seems reasonably consistent with our results. Therefore, we cannot say there is a smaller fraction of highly SFGs $\left(\mathcal{S F} \mathcal{R}_{\mathrm{TIR}}>10 \mathrm{M}_{\odot} \mathrm{yr}^{-1}\right)$ and/or dusty systems in the inner cores of clusters at intermediate redshifts.

Fig. 9 also displays different evolutions of $\mathcal{F}$ for clusters and field with $z$. The latter displays mild increasing trends for $\mathcal{F}_{\mathrm{UVJ}-\mathrm{SF}}$ and $\mathcal{F}_{\text {M-FIR }}$, which vary with $\beta=0.2 \pm 0.3$ and $0.2 \pm 0.5$, respectively. $\mathcal{F}$ remains $\sim 60$ percent for the $U V J-$ SF samples between $z=$ 0.19 and 0.89 . Flat/mild trends for the fraction of the star-forming population of galaxies in the field at intermediate redshifts $(z<1)$ are also found by Brammer et al. (2011) and Darvish et al. (2017). In particular, the latter gives 70 per cent of fraction of SFGs which is comparable with our results, although there is a larger offset between these numbers and the 40 percent given by the former. These differences are likely due to the sample selection criteria. The fraction of M-FIR galaxies remain also constant $(\sim 0.15)$ in the same redshift range. The decreasing trend of the data points at $z>0.570$ (not fitted) is due to the fact that the minimum $\mathcal{S} \mathcal{F} \mathcal{R}_{\mathrm{TIR}}$ detectable for this clusters is larger than the value used to select M-FIR galaxies.

If we now focus on the clusters, we can see that, despite the cluster-to-cluster variations (which reach $\sim 0.3$ ), we identify for both $U V J-$ SF and M-FIR samples a trend resembling the Butcher \& Oemler (1984) effect, in which the fraction of SFGs in clusters is observed to increase with redshift. In this case, the trends are fitted with $\beta=1.1 \pm 0.6$ and $7.3 \pm 5.8$ for the $U V J-S F$ and MFIR samples, respectively. The fraction of $U V J-S F$ galaxies within clusters increases from 28 percent at $z \sim 0.2$ to 47 percent at $z$ $\sim 0.9$, while the fraction of M-FIR galaxies grows from 0 per cent to 9 per cent in the same period. These values are in agreement with previous studies. For instance, Haines et al. (2009) find that the fraction of massive galaxies with $\mathcal{L}_{\mathrm{TIR}}>5 \times 10^{10} \mathcal{L}_{\odot}$ and $\mathcal{R}<R_{200}$ varies from $\sim 3$ per cent at $z=0.02$ to $\sim 10$ per cent at $z=0.3$ with $\beta=5.7_{-1.8}^{+2.1}$. The fraction varies between $\sim 1$ percent at $z=0.15$ and $\sim 4$ per cent at $z=0.3$ considering only $\mathcal{R} \lesssim 0.3 R_{200}$. Finally, the contribution of M-FIR galaxies to the whole SFGs population ( $U V J-S F$ sample) remains $\sim 23$ percent in the field, and varies from 0 percent to 19 percent in the clusters between $z \sim 0.2$ and 0.9. Martis et al. (2016) report very little evolution of the ratio of dusty and non-dusty SFGs as a function of stellar mass throughout this same redshift range.

The average values of $\mathcal{F}_{\mathrm{UVJ}-\mathrm{SF}}$ and $\mathcal{F}_{\mathrm{M}-\text { FIR }}$ do not present a clear trend with $\mathcal{R}$. In fact, all of them are compatible with the curve fitted to the fractions at $\mathcal{R} / R_{200}<0.1$. However, the distribution of SFGs in these high-density environments has been observed to increase with the projected cluster-centric radius by for example Alberts et al. (2016) and Haines et al. (2015). This could be the result of a combination of factors such as cluster to cluster variations and an intrinsic negligible trend with redshift at $\mathcal{R} / R_{200}<0.3$. 


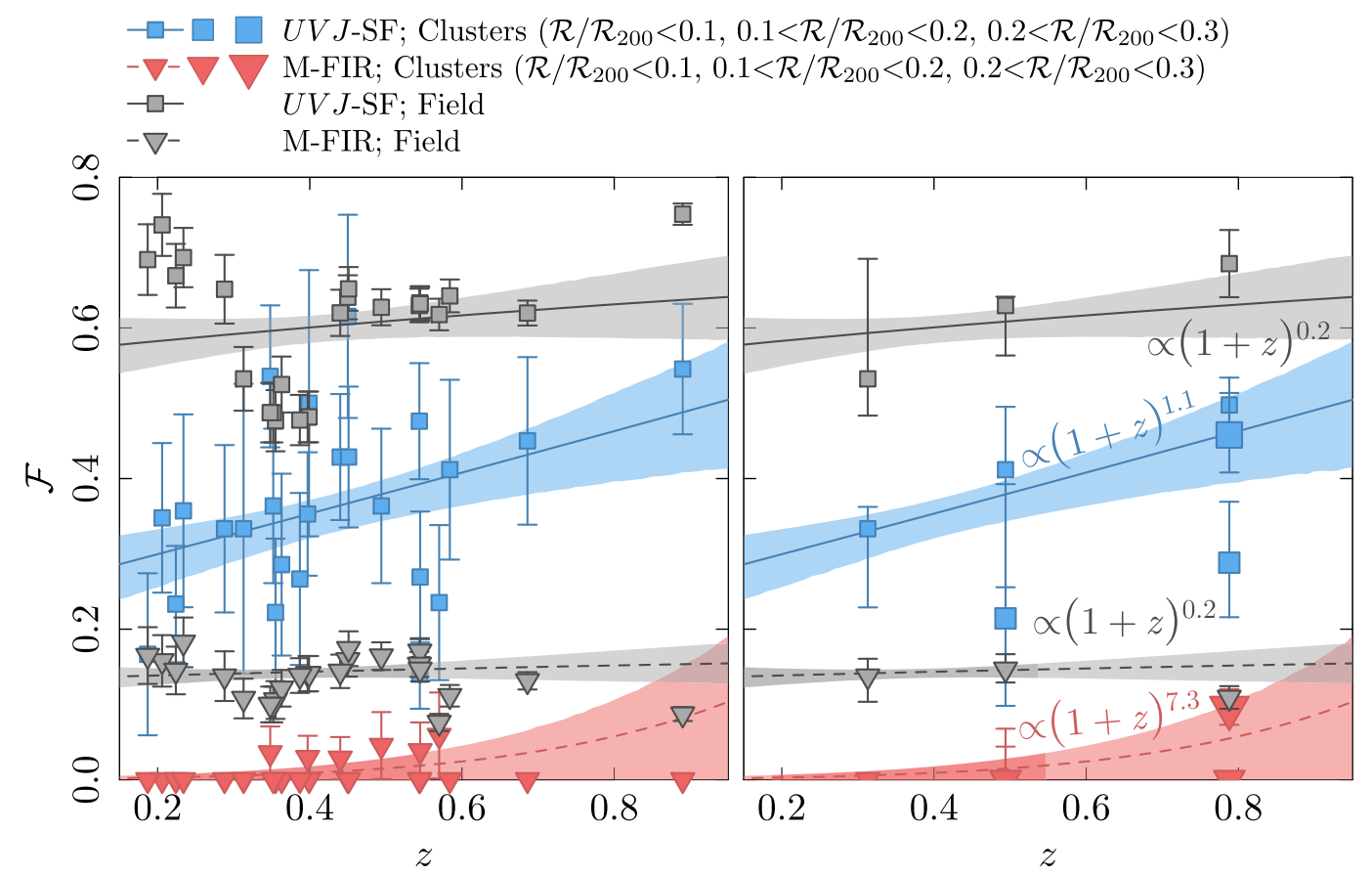

Figure $9 U V J-S F$ and M-FIR fractions. In the left-hand panel, we consider only cluster members at $\mathcal{R} / R_{200}<0.1$. In the right-hand panels, we show the average of the individual values at $\mathcal{R} / R_{200}<0.1,0.1<\mathcal{R} / R_{200}<0.2$, and $0.2<\mathcal{R} / R_{200}<0.3$, in three redshift bins $(0.2<z<0.4,0.4<z<0.6$, and $0.6<z$ $<0.9$ ). In both panels, we show the best fit of a trend with redshift of the shape $\alpha(1+z)^{\beta}$ and corresponding $1 \sigma$ confidence intervals (continuous and dashed lines, and corresponding shaded areas, respectively). Darker red shaded areas represent the redshift range used for the fit of the M-FIR samples of clusters and field. For each cluster for which the M-FIR sample is empty, we represent the corresponding data point on the bottom horizontal axis.

Table 10. Best-fitting parameters derived from the fit of the evolution with redshift of the $\mathcal{F}$, and median $\mathcal{S F} \mathcal{R}_{\mathrm{TOT}}$ and $s \mathcal{S F} \mathcal{R}_{\mathrm{TOT}}$ for all the $U V J-\mathrm{SF}$ and M-FIR samples in the clusters and field. For the clusters we include the results only for $\mathcal{R} / R_{200}<0.1$. The function fitted is a power law of the shape $\alpha(1+$ $z)^{\beta}$. The units of $\beta$ are $M_{\odot} \mathrm{yr}^{-1}$ and $\mathrm{yr}^{-1}$ in the case of the fit of $\mathcal{S F R}$ and $s \mathcal{S F R}$, respectively. The reduced $\chi^{2}$ for each case are shown in the last column. The fits of the $U V J-S F$ samples are performed using the data points spread out the whole redshift range. In the case of the M-FIR, we fit only reported redshift ranges.

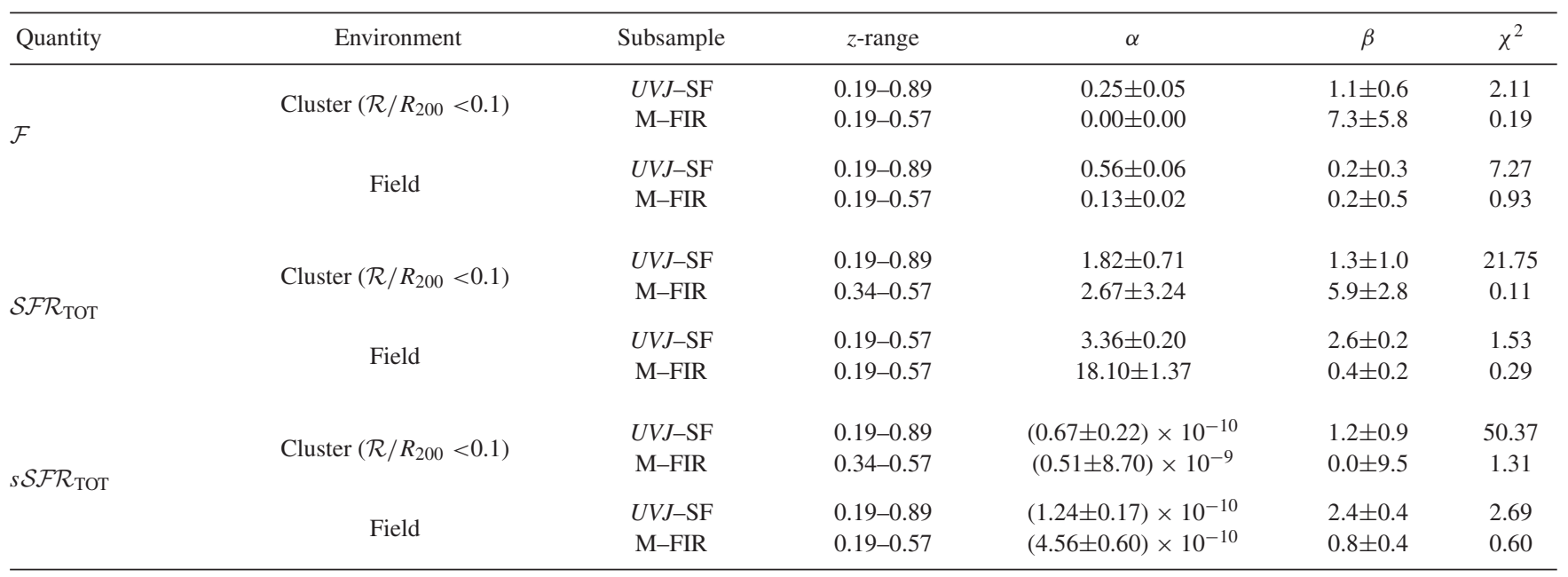

\subsection{Environmental quenching efficiency}

The environmental quenching efficiency $\left(\mathcal{Q} \mathcal{E}_{\text {env }}\right.$, van den Bosch et al. 2008; Peng et al. 2010; Balogh et al. 2016) is defined as

$\mathcal{Q} \mathcal{E}_{\text {env }}=\left(\mathcal{F}_{\mathrm{P}, \text { cluster }}-\mathcal{F}_{\mathrm{P} \text {,field }}\right) / \mathcal{F}_{\mathrm{SF}, \text { field }}$,

where $\mathcal{F}_{\mathrm{P} \text {,cluster }}$ and $\mathcal{F}_{\mathrm{P} \text {,field }}$ are the fraction of passive galaxies in the cluster and field, respectively, and $\mathcal{F}_{\mathrm{SF} \text {,field }}$ is the fraction of SFGs in the field.
In Fig. 10, we show the $\mathcal{Q} \mathcal{E}_{\text {env }}$ in the cluster cores $\left(\mathcal{R} / R_{200}<0.1\right)$ grouped in three redshift bins $(0.2<z<0.4,0.4<z<0.6$, and $0.6<z$. $<0.9$ ). We derive $\mathcal{Q} \mathcal{E}_{\text {env }}$ values of $0.49_{-0.08}^{+0.09}, 0.38_{-0.07}^{+0.08}$, and $0.30_{-0.08}^{+0.07}$ at $z \sim 0.31,0.49$, and 0.79 , respectively. These values are smaller than those presented by Nantais et al. (2017) at $0.87<z<1.63$ for galaxies with $\log _{10} \mathcal{M}_{*} / \mathrm{M}_{\odot} \geq 10.3$. Our value of $\mathcal{F}_{\mathrm{UVJ}-\mathrm{SF}}$ for clusters (field) in the highest redshift bin is $0.50_{-0.03}^{+0.03}\left(0.69_{-0.04}^{+0.04}\right)$ which leads to smaller values of the passive fraction than their $0.88_{-0.03}^{+0.04}$. Our results at $z \sim 0.8$ are also smaller than other works 


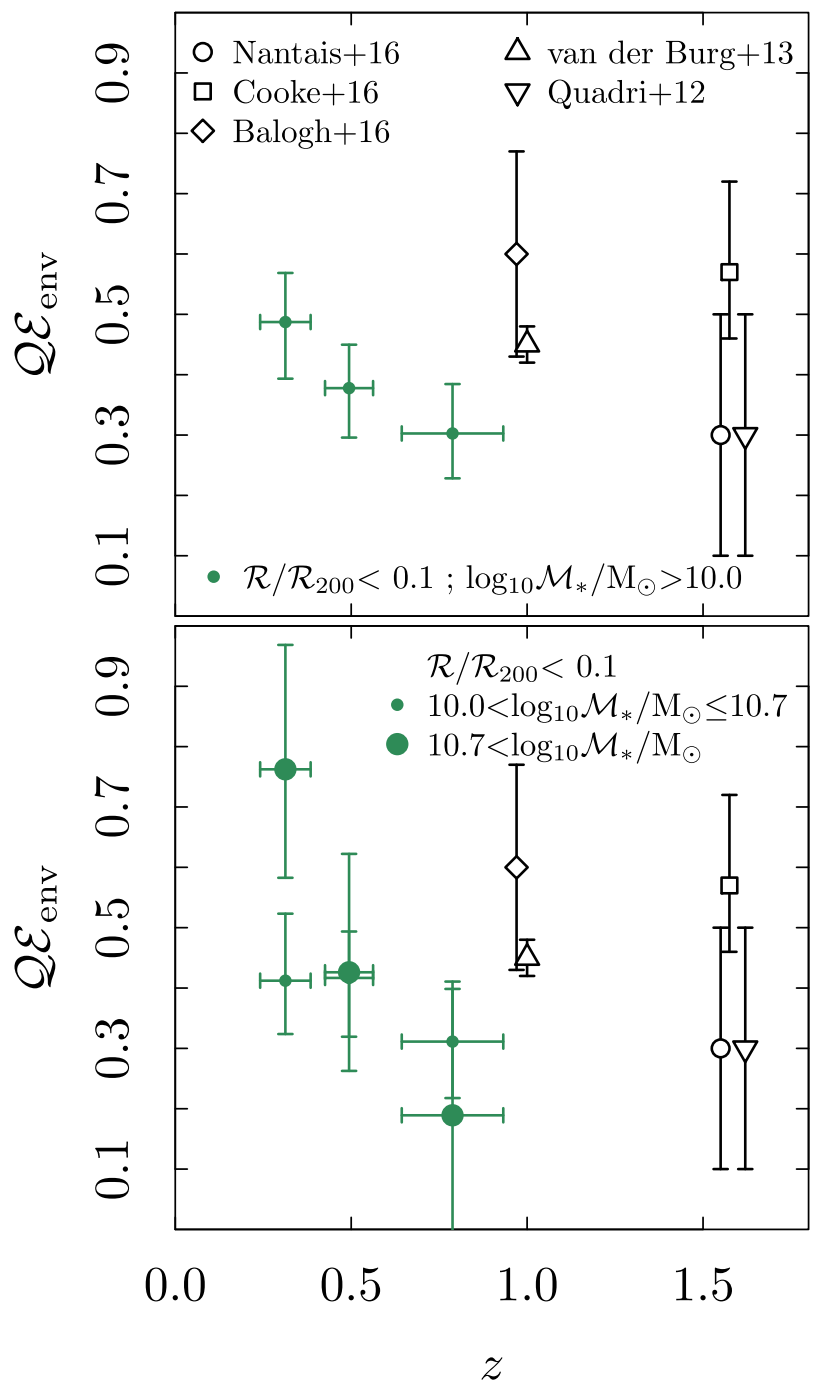

Figure 10 Top panel: environmental quenching efficiency for galaxies with $\log \mathcal{M}_{*} / \mathrm{M}_{\odot}>10$ in three $z$ bins. Bottom panel: environmental quenching efficiency calculated for two mass bins $\left(10.0<\log _{10} \mathcal{M}_{*} / \mathrm{M}_{\odot} \leq 10.7\right.$ and $\left.\log _{10} \mathcal{M}_{*} / \mathrm{M}_{\odot}>10.7\right)$. In all cases, error bars are calculated propagating the errors of the fractions, which were obtained through bootstrap (500 realizations) in the initial cluster members and field galaxies samples. For comparison purposes, we include the $\mathcal{Q} \mathcal{E}_{\text {env }}$ values given by Quadri et al. (2012), van der Burg et al. (2013), Balogh et al. (2016), Cooke et al. (2016), and Nantais et al. (2016).

such as Balogh et al. (2016) at redshift $z \sim 1$ for the same values of stellar mass. It is worth noting that these works calculate the $\mathcal{Q} \mathcal{E}_{\text {env }}$ within cluster-centric distances of $1 \mathrm{Mpc}$ or $R_{200}$, while we focus on the inner cluster core, where the fraction of passive galaxies is expected to be larger.

The dependence of the $\mathcal{Q} \mathcal{E}_{\text {env }}$ with stellar mass is under debate. While some works (e.g. Peng et al. 2010; van der Burg et al. 2018) claim environmental quenching to be independent of mass quenching, others (e.g. Lin et al. 2014; Kawinwanichakij et al. 2017) have detected an increasing trend of the $\mathcal{Q} \mathcal{E}_{\text {env }}$ with stellar mass. The bottom panel of Fig. 10 shows the values of $\mathcal{Q} \mathcal{E}_{\text {env }}$ obtained for galaxies at $\mathcal{R}<0.1 R_{200}$ in two stellar mass bins $\left(10.0<\log _{10} \mathcal{M}_{*} / \mathrm{M}_{\odot}<10.7\right.$ and $\left.10.7<\log _{10} \mathcal{M}_{*} / \mathrm{M}_{\odot}\right)$. As we can see, only in the first redshift bin the $\mathcal{Q} \mathcal{E}_{\text {env }}$ appears significantly larger for the more massive galaxies. This $\mathcal{Q} \mathcal{E}_{\text {env }}$ appears larger also if we split the sample at lower masses, but the significance of the result decreases. Darvish et al. (2016) claim that environmental quenching efficiency is almost independent of stellar mass at $z$ $<1$, except for galaxies with $\log _{10} \mathcal{M}_{*} / \mathrm{M}_{\odot}>10.9$, that high-density environments could quench more efficiently.

\subsection{Average $\mathcal{S F R}$ and $s \mathcal{S F R}$}

A complementary quantification of the SF activity in clusters tackles the question whether beyond the decrease in $\mathcal{F}$ shown in Fig. 9, the impact of the cluster environment modifies the distribution of the rates at which the remaining SFGs form stars. In Fig. 11 (top and bottom left hand panels), we display, as a function of redshift, the median $\mathcal{S F R}$ and $s \mathcal{S F R}$ of each cluster $\left(\mathcal{R} / R_{200}<0.1\right)$ and field sample of $U V J-S F$ and M-FIR galaxies. The error bars are determined using the bootstrap technique to derive the $1 \sigma$ confidence intervals, and thus, they represent the spread in the $\mathcal{S F R}$ and $s \mathcal{S F R}$ of each subsample, not the intrinsic error of the estimation of these parameters $(\sim 0.3 \mathrm{dex})$. In the corresponding right-hand panels, we display the median values and confidence intervals in three redshift bins. We also include the median values obtained at $0.1<\mathcal{R} / R_{200}<0.2$ and $0.2<\mathcal{R} / R_{200}<0.3$, when possible.

To quantify the trends of the average $\mathcal{S F R}$ and $s \mathcal{S F R}$ with redshift, we again fit the median values (of the individual clusters) using a function of the shape $\alpha(1+z)^{\beta}$. Regarding the M-FIR samples, we only fit those data points corresponding to clusters at $z<0.57$ where at least a galaxy is detected in the MIR and/or FIR. Effectively, the fit is performed only between $0.34<z<0.57$ (darker shaded area in Fig. 12). The best-fitting parameters are shown in Table 10. We also include a corresponding $1 \sigma$ confidence intervals of the fit (generated using Monte Carlo simulations) as a shaded area around each best-fitting curve. The confidence intervals are not representative of the dispersion of the $\mathcal{S F R}$ and $s \mathcal{S F R}$ distributions, typically $\sim 0.3$ dex.

Regarding the $U V J-$ SF samples, Fig. 11 clearly shows an offset between the field and the clusters, with the latter displaying $\mathcal{S F R}$ and $s \mathcal{S F R}$ on average $\sim 0.3$ dex lower. This offset cannot be explained by the differences between the mass distribution of field and clusters samples (see Section 8). This can be seen in Figs 5 and 6, where the offsets in $\mathcal{S F R}$ and $s \mathcal{S F R}$ are visible at fixed $\mathcal{M}_{*}$.

Fig. 11 also displays a clear increasing trend with $z$ of the $\mathcal{S F R}$ for both field and cluster $U V J-S F$ samples $(\beta=2.6 \pm 0.2$ and $1.3 \pm 1.0$, respectively). The average $\mathcal{S F R}$ and $s \mathcal{S F R}$ do not show a strong differential evolution relative to the field but a systematic offset. Analogous trends are found for the $s \mathcal{S F R}$, with $\beta=2.4 \pm 0.4$ and $1.2 \pm 0.9$ for the field and the clusters, respectively. This also suggests that there is not a significant evolution of the $\mathcal{M}_{*}$ distributions driving the variation in $s \mathcal{S F R}$, at least at $\log _{10} \mathcal{M}_{*} / M_{\odot}>10$. A hypothetical impact of the stellar mass distributions of the cluster and field samples would translate into a different behaviour of the variation of the average values of $\mathcal{S F R}$ and $s \mathcal{S F R}$ with environment, which is something we do not observe.

The high cut in $\mathcal{S F} \mathcal{F}_{\mathrm{TIR}}$ we use to build the M-FIR galaxy samples translates into a mild increasing trend with $z$ of the median value of the average $\mathcal{S F R}(s \mathcal{S F R})$ for the M-FIR galaxies in the field, which varies with $\beta=0.4 \pm 0.2(0.8 \pm 0.4)$. Within the cluster cores, we derive field-like values of $\mathcal{S F R}$ and $s \mathcal{S F R}$. Also, due to the mentioned $\mathcal{S F} \mathcal{R}_{\mathrm{TIR}}$ constraint we are not able to explore whether the M-FIR samples behave in the same way as the 

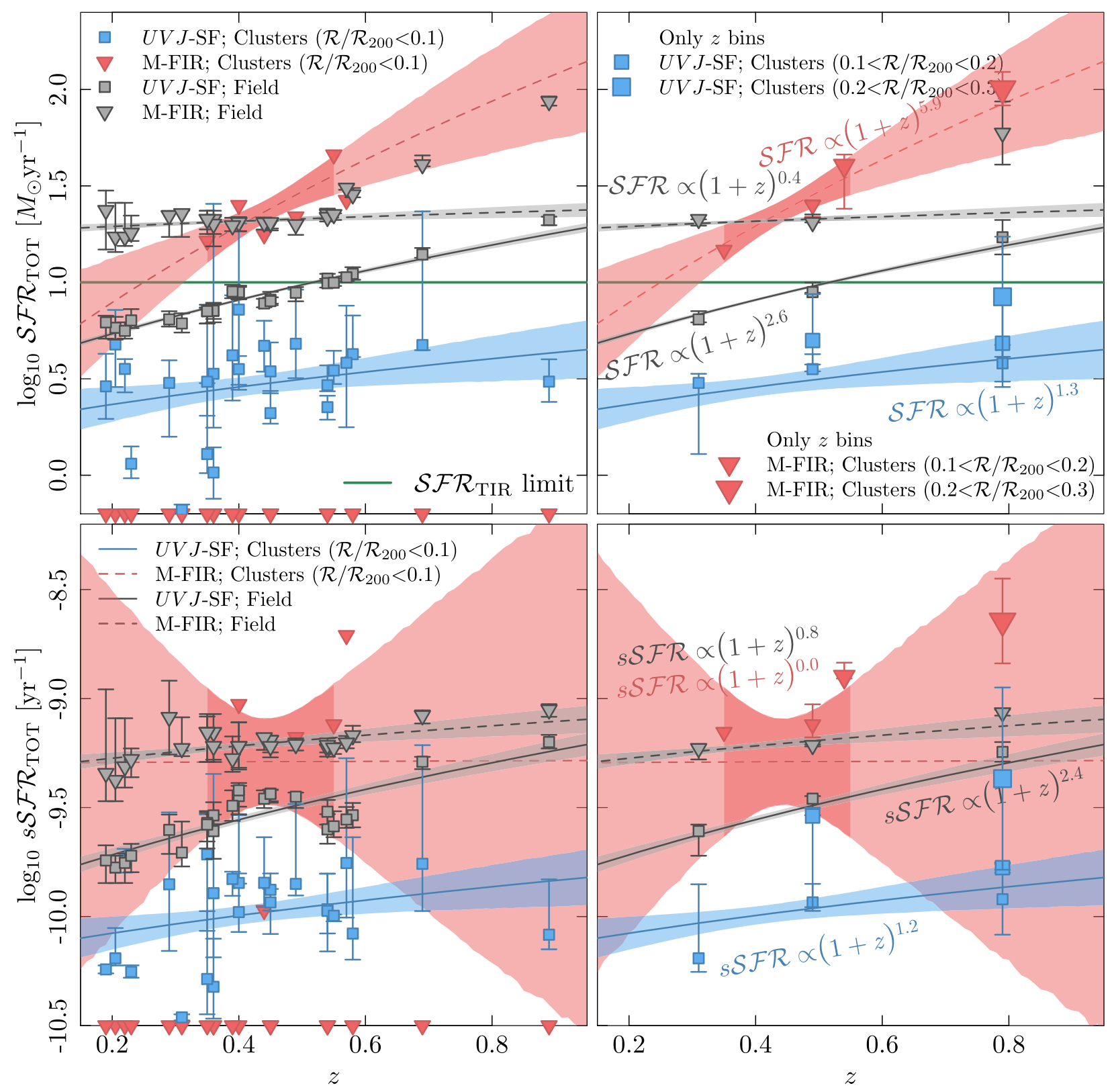

Figure 11 Top panel: median $\mathcal{S F R}$ for the $U V J-\mathrm{SF}$ and M-FIR samples. Bottom panel: median $s \mathcal{S F \mathcal { R }}$ TOT for the $U V J-\mathrm{SF}$ and M-FIR samples. Representation as in Fig. 9.

UVJ-SF samples. The M-FIR galaxies with suppressed SF are simply missed by the selection function.

A number of works have also identified an offset between the average $\mathcal{S F R}$ and $s \mathcal{S F R}$ in the clusters and field (e.g. Patel et al. 2009; Vulcani et al. 2010; Haines et al. 2013, 2015; Paccagnella et al. 2016). Among them, Alberts et al. (2014) find that blue cluster galaxies $\left(\mathcal{M}_{*} \geq 1.3 \times 10^{10} M_{\odot}\right)$ present systematically lower average $s \mathcal{S F} \mathcal{R}_{\mathrm{TIR}}$ up to $z \sim 1.4$. Their results, derived through a stacking analysis on Herschel/SPIRE $250 \mu \mathrm{m}$ imaging of 270 massive galaxy clusters between $z \sim 0.3$ and 1.5 , quantify the average level of SF of the whole star-forming cluster galaxy population, rather than the typical rate of SF of FIR-detected galaxies. In fact, the average $s \mathcal{S F R}$ they retrieve for clusters at $z \sim 0.5$ and $\sim 0.8(\sim-9.70$ and $\sim-9.50$, respectively) are comparable with ours, as well as their $0.2-0.3$ dex differences with the field. This systematic suppression of the level of star-forming activity within rich environments is created by the existence of a numerous population of transition galaxies located in the lower part of the well-studied MS of SFGs (e.g. Paccagnella et al. 2016; Coenda, Martínez \& Muriel 2018). Also, Haines et al. (2013) find a 0.2 dex suppression of the $s \mathcal{S F R}$ in SFGs with $\log _{10} \mathcal{M}_{*} / M_{\odot}>10$ and $\mathcal{S F R}>3 M_{\odot} \mathrm{yr}^{-1}$ within $\mathcal{R}_{200}$ at $0.15<z<0.3$.

If we now focus on the trend with $\mathcal{R}$ in the two last redshift bins, we can see how the average $\mathcal{S F R}$ and $s \mathcal{S F R}$ increase significantly for $U V J-\mathrm{SF}$ galaxies at $0.2<\mathcal{R} / R_{200}<0.3$, reaching field-like values. This is probably due to the fact that we are reaching the region slightly beyond $0.3 R_{200}$, where most of the prototypes of galaxies violently interacting with the ICM are found (e.g. jellyfish galaxies, Poggianti et al. 2016; see Boselli \& Gavazzi 2006, and references therein). The average values of $\mathcal{S F R}$ for the 

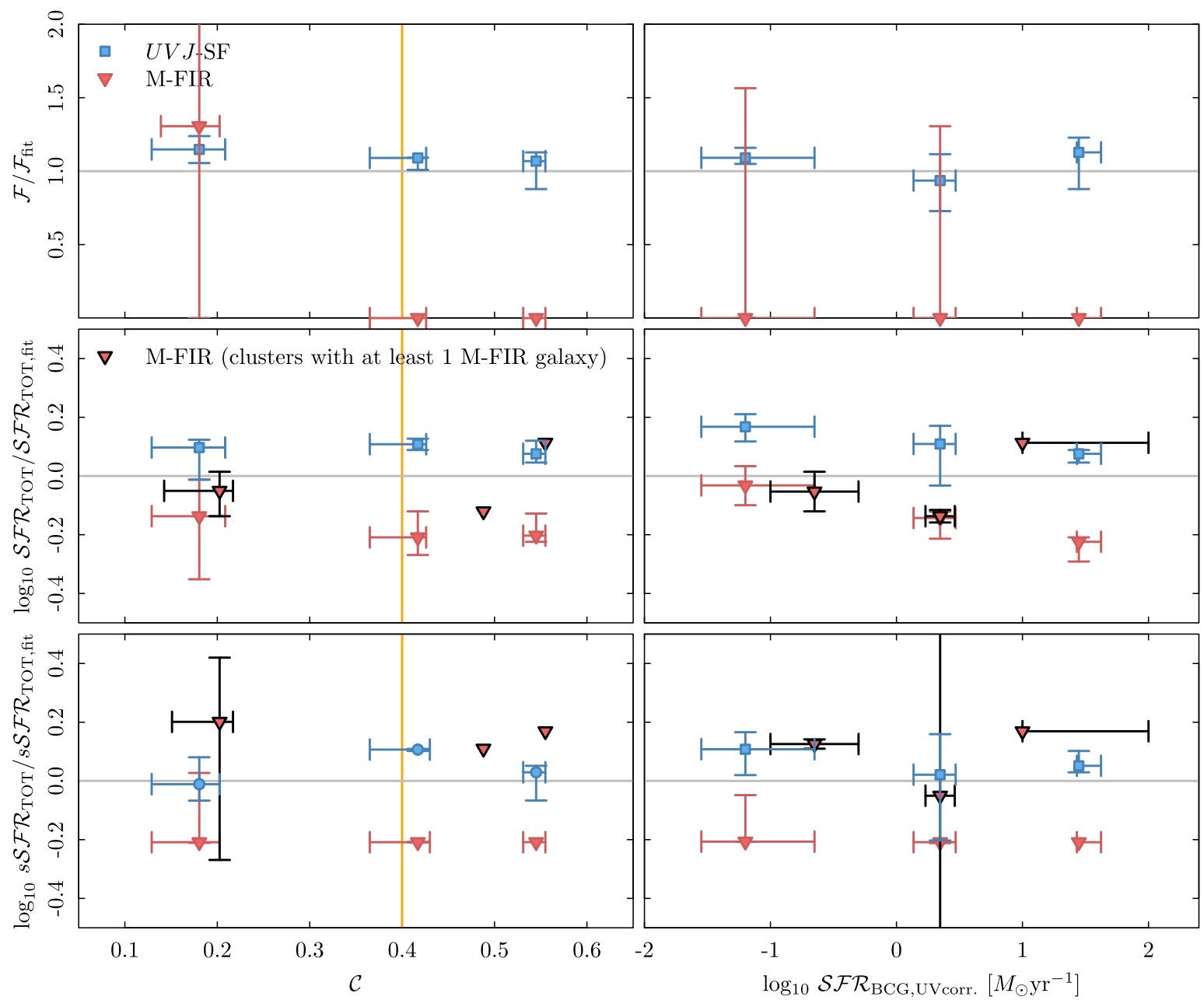

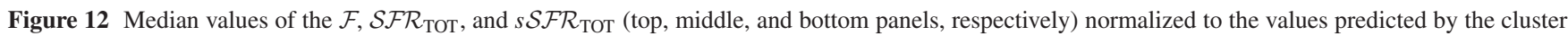
trends in Figs 9 and 11 at the corresponding redshifts versus the $\mathcal{C}$ coefficient given by Donahue et al. (2016, indicator of the presence of a CC; left-hand panels), and the $\mathcal{S F} \mathcal{R}_{\mathrm{UV}}$ of the BCG (right-hand panels; Fogarty et al. 2015) corrected for extinction. The vertical yellow line on the left-hand panels represent the value of $\mathcal{C}$ over which the $\mathrm{CC}$ are normally located (Donahue et al. 2016). We present the averaged values in three equally populated bins of each $x$-axis parameter. Values derived for the UVJ-SF and M-FIR samples are shown with blue and red symbols, respectively. We use circles to represent the results including all the clusters. In the case of the clusters where no galaxy was selected in the MIR/FIR we use an average $\mathcal{S F} \mathcal{R}_{\mathrm{TOT}}=10 M_{\odot} \mathrm{yr}^{-1}\left(\right.$ the $\mathcal{S F} \mathcal{R}_{\mathrm{TIR}}$ limit of our study), and an average $\log _{10} s \mathcal{S F R}$ TOT $=-9.5$. We use triangles to represent the averages found using only clusters with obscured $\mathrm{SF}$ activity in their core (at least 1 MIR/FIR-detected galaxy).

cluster M-FIR remain overall compatible with the field values. Instead, the $s \mathcal{S F R}$ depart from the field trend at larger $\mathcal{R}$. However, limited number counts of this sample do not allow to extract robust conclusions about this sample.

\subsection{Star formation dependence on individual cluster properties: cool-core and BCG's star formation}

In the previous subsections, we have analysed the SF properties of $\mathcal{M}_{*}$-limited samples of star-forming cluster members detected and undetected in the MIR and/or FIR. Even though we are able to identify a trend of the SF indices with redshift, the scatter in the average properties is large. These cluster-to-cluster variations have been observed frequently in the past, and some works have attempted to quantify them (e.g. Alberts et al. 2016). This scatter is likely due to a combination of stochastic processes, such as galaxy mergers (probably, the limited area covered by our study worsens this effect), and differences in the properties of the clusters, such as the dynamical state (e.g. Stroe et al. 2015). In this section, we aim at exploring this latter.

Despite the fact of being selected to be largely relaxed, there is disagreement in the literature on the dynamical state of CLASH sample members (see Rumsey et al. 2016, and references therein). Given that we are focusing our study on the inner cores of clusters, we use as a proxy of the dynamical state of these systems the presence of a CC and the SF activity undergone by their BCGs. Rawle et al. (2012a) found these observables to be strongly correlated, which suggests that the SF activity of the BCGs is influenced by the cluster-scale cooling process. In fact, star-forming BCGs seem to be exclusively found in the centres of $\mathrm{CC}$ clusters. However, the separation between $\mathrm{CC}$ and not-CC clusters is challenging. In this work, we use as an indicator of the presence of this feature the parameter $\mathcal{C}$, as defined by Donahue et al. (2016), which is a measure of the concentration of the X-ray emission. More precisely, it gives the ratio between the light within a circular aperture with a $100 \mathrm{kpc}$ radius and the total light enclosed within a circular aperture with a $500 \mathrm{kpc}$ radius. For $\mathrm{CC}$ clusters, $\mathcal{C}$ values are likely $>0.4$ (Donahue 
et al. 2016). Among the 24 CLASH+HLS clusters, 12 qualify this criterion. As we previously mentioned we find eight MIR/FIRemitter BCGs. Two of them already identified by Rawle et al. (2012a, the remaining six are not included in their sample). Among the eight, seven are characterized by $\mathcal{C}>0.4\left(\mathcal{C}_{\mathrm{AS} 1063}=0.19 \pm 0.03\right)$. In turn, the formation of a $\mathrm{CC}$ appears also to be linked to the dynamical states of the clusters, with relaxed clusters exhibiting more likely $\mathrm{CC}$ than unrelaxed systems. Although some works have identified distant clusters hosting a $\mathrm{CC}$, their strength at $z$ $>0.7$ appears significantly lower due to the expected higher cluster merger rate and their more immature evolutionary state (Santos et al. 2008).

Fig. 12 displays, for both the UVJ-SF and the M-FIR samples $\left(\mathcal{R} / R_{200}<0.1\right)$, the relation between the three quantities we use to analyse the $S F$ activity in clusters (i.e. $\mathcal{F}, \mathcal{S F R}$, and $s \mathcal{S F R}$ ) and both the parameter $\mathcal{C}$ and the $\mathcal{S F} \mathcal{R}_{\mathrm{UV}}$ of the BCGs extinction corrected $\left(\mathcal{S} \mathcal{F} \mathcal{R}_{\mathrm{UV} \text { corr,BCG}}\right)$ provided by Fogarty et al. (2015). In order to remove the global trends with redshift of the average $\mathcal{F}, \mathcal{S F R}$, and $s \mathcal{S F R}$ that could have an impact on the results, we remove them by normalizing these quantities to the values predicted by the trends fitted for the clusters in the previous subsection at the corresponding redshifts. In each panel of Fig. 12, we show the median in three bins of the corresponding $x$-axis parameter populated by the 33 per cent of the clusters sample. Error bars represent the confidence intervals derived through a bootstrap methodology. In the case of the MFIR samples, we show with highlighted triangles (black border) the median values of the clusters which contain at least one object. We use red triangles for the medians calculated considering upper limits $\mathcal{S F R}=10 M_{\odot} \mathrm{yr}^{-1}$ (our $\mathcal{S F} \mathcal{R}_{\mathrm{TIR}}$ limit for the M-FIR samples) and $s \mathcal{S F} \mathcal{R}_{\text {TOT }}=3 \times 10^{-10} \mathrm{yr}^{-1}$ for those clusters where no M-FIR galaxy is found.

If we focus on the upper panels of Fig. 12, we see that the bins of larger values of $\mathcal{C}$ and $\log _{10} \mathcal{S} \mathcal{F} \mathcal{R}_{\mathrm{UV} \text { corr,BCG }}$ are marginally dominated by less MIR/FIR-detected SFGs. However, the large error bars make the trend not significant. In the middle and bottom panels, we do not find a clear correlation between the average $\mathcal{S F R}$ or the $s \mathcal{S F R}$ and either $\mathcal{C}$ or $\log _{10} \mathcal{S} \mathcal{F} \mathcal{R}_{\mathrm{UV} \text { corr,BCG}}$.

\section{DISCUSSION}

It has long been claimed that galaxies quench more efficiently in clusters than in the field (e.g. Butcher \& Oemler 1984; Gerke et al. 2007; Haines et al. 2009, 2013). The general interpretation of this suppression of SF is that environmental processes favour the removal of gas reservoirs from galaxies. In fact, this average deficit of gas in cluster members has been observationally confirmed in star-forming cluster spirals by, for example Jablonka et al. (2013). In agreement with this framework, our results clearly display a lack of SF activity in massive cluster cores with respect the field at intermediate redshifts in terms of both the fraction of SFGs and the rates at which they form stars.

The observed significant systematic $\sim 0.3$ dex offsets between clusters and field average $\mathcal{S F R}$ and $s \mathcal{S F R}$ do not appear to be the result of differences in the SMF of the galaxy samples studied. Supporting this, Guglielmo et al. (2015) find that galaxies of a given mass have different SFHs depending on their environment, and therefore, it is not the distributions of galaxy masses in clusters the origin of the observed dependence of the SF with the environment. Given that the population of SFGs within massive clusters at the intermediate redshifts probed is thought to be dominated by infalling field galaxies (Kauffmann 1995), if the quenching of these galaxies were dominated by the same processes that turn galaxies off in the field (leading to the global SF decline in the Universe since $z \sim 1-2$, Madau \& Dickinson 2014) the fraction of SFGs should decrease proportionally in both environments (Haines et al. 2009). Given the different evolution with redshift, we derive for $\mathcal{F}_{\mathrm{UVJ}-\mathrm{SF}}$ in clusters and field, we can say that we are witnessing the imprint of the impact of environment on the evolution of cluster galaxies $\left(\mathcal{M}_{*}>10^{10} \mathrm{M}_{\odot}\right)$.

Our results appear to support the observed evolution of the environmental quenching efficiency (van den Bosch et al. 2008; Peng et al. 2010; Balogh et al. 2016), defined as the fraction of passive cluster galaxies which would be still star-forming if they were in the field (Nantais et al. 2017), with a major rise since $z \sim 2$ (e.g. Butcher \& Oemler 1984; Gerke et al. 2007; Haines et al. 2009, 2013; Alberts et al. 2016).

It is straightforward to wonder what are the processes intrinsic to high-density environments that drive the aforementioned galaxy transformation. Some of the most commonly invoked are: strangulation (Larson et al. 1980), which consists on the removal of the loosely bound hot halo gas reservoirs by the ICM on long time-scales $(>1 \mathrm{Gyr})$; the removal of the ISM through interactions with the ICM on moderate/short time-scales ( $\lesssim 1$ Gyr) RPS (Gunn \& Gott 1972; Steinhauser, Schindler \& Springel 2016); either galaxy-galaxy or galaxy-cluster gravitational interactions, grouped together under the name harassment (Moore et al. 1996). The SFGs infalling into high-density environments at $z \lesssim 1$ are very likely influenced by a combination of these dynamical gas removal processes (see Boselli \& Gavazzi 2006; Vulcani et al. 2016). Merger events are probably less frequent in cluster cores at these redshifts, where the high relative velocities hamper reaching the fraction of encounters observed in the field. However, there is growing evidence (e.g. Brodwin et al. 2013; Lotz et al. 2013; Santos et al. 2015; Alberts et al. 2016; Balogh et al. 2016) that at higher redshifts, mergers play the major role in quenching infalling SFGs due to high galaxy space densities and low relative velocities (e.g. Brodwin et al. 2011).

The small scatter $(\sim 0.3 \mathrm{dex})$ found for the MS of SFGs in field samples (e.g. Noeske et al. 2007, Renzini \& Peng 2015) is usually interpreted as the consequence of a quenching mechanism that is capable of moving rapidly ( $0.1 \mathrm{Gyr}$ time-scales) the galaxies out (downward) of the MS. For this reason, the downward offset of the MS found in our work and in other previous studies in clusters (e.g. Haines et al. 2013, Paccagnella et al. 2016) has frequently been interpreted as the imprint of different environmentally driven quenching mechanisms that could turn-off infalling SFGs slowly (e.g. Haines et al. 2013), thus, populating the region below the MS with galaxies on their way to be quenched. The work by Haines et al. (2015), based on the analysis of the actual orbits of infalling galaxies in the 75 most massive clusters in the Millennium Simulation (Springel et al. 2005) support the slow quenching scenario with time-scales $\sim 0.7-2$ Gyr. The most frequently proposed mechanism for slow quenching in high-density environments is strangulation. In this evolving scenario, the decline in SF is very likely due to overconsumption (McGee, Bower \& Balogh 2014), the exhaustion of a gas reservoir through SF and expulsion via modest outflows in the absence of cosmological accretion. Maier et al. (2016) also propose it as the explanation for the higher metallicities found in the accreted cluster galaxies of MACS0416. It has also been invoked to explain the increasing distribution of SFGs with the projected cluster-centric radius (e.g. Haines et al. 2015; Alberts et al. 2016).

However, numerous studies have found observational evidence of rapid quenching mechanisms, such as RPS, that can remove the 
gas of an infalling galaxy in time-scales of the order of the cluster crossing time ( $\lesssim 1$ Gyr, e.g. Wetzel et al. 2013), playing a significant role building the populations of passive galaxies in clusters at different redshifts. Also, some models of galaxy strangulation (e.g. Boselli et al. 2016, and references therein) and numerical simulations (e.g. McGee et al. 2014) predict extremely long timescales in order to reproduce the observed lack of SF activity in cluster members, while for instance Boselli et al. (2016) claim that only RPS is able to significantly quench SF activity in galaxies perturbed by high-density environments. The contribution of RPS in the core of clusters is thought to be important given the high relative velocities and higher densities of the ICM (e.g. Gunn \& Gott 1972). However, this phenomenon operates efficiently for extreme cases of infall in which the orbital velocity is particularly high and the galaxy inclination is perpendicular to the direction of motion (Abadi, Moore \& Bower 1999). Furthermore, RPS can present a fluctuating behaviour which means that galaxies suffering from stripping can present a wide range of properties, as observed by Vulcani et al. (2016, 2017).

As an alternative to the slow/fast dichotomy frequently discussed, Wetzel et al. (2013) propose a delayed-then-rapid quenching scenario, in which the satellites $\mathcal{S F R s}$ evolve unaffected for 2-4 Gyr after infall, and are eventually quenched rapidly, with an e-folding time of $<0.8$ Gyr. This scenario has been frequently embraced to conciliate the observations of smaller fractions of SFGs in clusters and values of $\mathcal{S F R}$ comparable to those in the field at the same redshift.

In addition, Wetzel et al. (2013) propose the quenching timescales do not depend on the halo mass. Interestingly, they claim that up to half of quenched satellites in massive clusters is the result of quenching in infalling groups, namely, pre-processing. Other authors have highlighted the importance of this phenomenon to explain the properties of galaxy populations of intermediateredshift clusters (e.g. Haines et al. 2015; Ogrean et al. 2015). The cluster-centric distances we probe in this work $\left(\mathcal{R} / R_{200}<0.3\right)$ do not allow the assessment of pre-processing.

In this context, our results favour slow quenching mechanisms (e.g. strangulation) to be dominating the evolution of the observed $U V J-$ SF cluster core galaxies with $\log _{10} \mathcal{M}_{*} / M_{\odot}>10$ throughout the last $8 \mathrm{Gyr}$. This is because these samples appear to be heavily populated by transition galaxies observed while they quench (Paccagnella et al. 2016). However, we cannot rule out the contribution of fast processes such as RPS to the enhanced fraction of quenched galaxies observed. We also note that our methodology cannot directly select galaxies quenching on short time-scales, such as PSB (e.g. Poggianti et al. 2004; Tran et al. 2007; Muzzin et al. 2014; Paccagnella et al. 2017), as this would require spectral information, which we lack for more than half of our clusters sample.

\section{SUMMARY AND CONCLUSIONS}

We have presented a detailed analysis of the SF activity within 24 massive clusters cores at $0.2 \lesssim z \lesssim 0.9$ targeted by the HLS and CLASH surveys. The deep multiwavelength photometric data set on these fields cover the whole rest-frame UV-to-FIR regimes. In particular, we have made use of the CLASH catalogues, which contain photometry measured on HST ACS/WFC (F435W, F475W, F606W, F625W, F775W, F814W, and F850LP), WFC3/UVIS (F225W, F275W, F336W, and F390W), and WFC3/IR (F105W, F110W, F125W, F140W, and F160W) imaging. Then, we have combined these catalogues with others built on Spitzer/ IRAC (3.6, 4.5, 5.8, and $8.0 \mu \mathrm{m})$ and MIPS $(24 \mu \mathrm{m})$ bands, and Herschell PACS (100 and $160 \mu \mathrm{m})$ and $\operatorname{SPIRE}(250,350$, and $500 \mu \mathrm{m})$, deblending the former in the position of the CLASH catalogues and selecting the most probable UV/optical counterpart for the sources in the MIR and FIR bands. Finally, we have also gathered the spectroscopic informations available on these fields, mainly released by CLASH-VLT and GLASS surveys. Consequently, we have derived high-quality photometric redshifts $\left(\sigma_{\mathrm{NMAD}}=0.04\right.$, and 8 percent of outliers) fitting the UV-to-NIR photometry with the EAZY code. We have selected cluster members by applying either a spectroscopic redshift criterion or a probabilistic methodology that takes into account the whole information included in the $\mathcal{P}(z)$ of the photometric redshift estimation. We have used the $z_{\text {phot }}$ derived and the RAINBOW Cosmological Database software package to fit, on the one hand, the optical/NIR photometry (CLASH and Spitzer/IRAC), and on the other hand, the MIR/FIR photometry (Spitzer/MIPS and Herschel). In this way, we have estimated the physical properties of the cluster members such as their $\mathcal{M}_{*}$ and the rates at which they form stars (as traced by the UV and FIR emission independently). With the aim of building up analogous field samples with which compare the results on clusters, we have applied the same analysis and selection criteria on three CANDELS fields. Finally, we have used samples of SFGs $\left(\mathcal{M}_{*}>10^{10} M_{\odot}\right)$ selected using the $U V J$ diagram ( $U V J-\mathrm{SF}$ samples) to evaluate and compare the SF processes in high-density environments and the field. Furthermore, we have used samples of galaxies $\left(\mathcal{M}_{*}>10^{10} M_{\odot}\right)$ detected in the MIR and/or FIR with $\mathcal{S F} \mathcal{F}_{\mathrm{TIR}}>10 M_{\odot} \mathrm{yr}^{-1}$ (M-FIR samples) to explore the obscured SF activity. Taking advantage of the rich data set available, we have based our results on the quantification of the total SF, defined as either the sum of the SF traced by the rest-frame UV emission and the FIR, or the unobscured SF (traced only by the rest-frame UV) corrected for the dust extinction with our own optimized recipe.

The main results and conclusions of our work can be summarized in the following points:

(i) The SF activity in the inner regions of intermediate- $z$ clusters appears to be suppressed in terms of both the fraction of SFGs and the rate at which they turn gas into stars.

(ii) We derive average fractions of $U V J-\mathrm{SF}$ galaxies a factor $\sim 2$ smaller in cluster $\left(\mathcal{R} / R_{200}<0.1\right)$ than in the field across. The average fraction of M-FIR cluster members $\left(\mathcal{R} / R_{200}<0.1\right)$ is negligible but compatible with a factor $\sim 2$ smaller in clusters.

(iii) We identify increasing trends of $\mathcal{F}_{\mathrm{UVJ}-\mathrm{SF}}$ and $\mathcal{F}_{\mathrm{M}-\mathrm{FIR}}$ with $z$, which evolve faster within clusters $(\beta=1.1 \pm 0.6$ and $7.3 \pm 5.8$, respectively, at $\left.\mathcal{R} / R_{200}<0.1\right)$ than in the field $(\beta=0.2 \pm 0.3$ and $0.2 \pm 0.5$, respectively).

(iv) $U V J-S F$ cluster members $\left(\mathcal{R} / R_{200}<0.1\right)$ present $\mathcal{S F R}$ and $s \mathcal{S F R}$ typically $\sim 0.3$ dex smaller than $U V J-S F$ field galaxies. Average $\mathcal{S F R}$ and $s \mathcal{S F R}$ values evolve similarly (within the error bars) in clusters, with $\beta=1.3 \pm 1.0$ and $1.2 \pm 0.9$, respectively. The evolution in the field is described by $\beta=2.6 \pm 0.2$ and $2.4 \pm 0.4$, respectively. Due to the high $\mathcal{S} \mathcal{F} \mathcal{R}_{\mathrm{TIR}} \mathrm{s}$ completeness value given Spitzer/MIPS $24 \mu \mathrm{m}$ and Herschel imaging used in this study, we cannot explore whether is there a different trend between field and clusters dusty SFGs in the average $\mathcal{S F R}$ and $s \mathcal{S F R}$.

(v) We find increasing SF activity with cluster-centric distance out to $\mathcal{R} / R_{200}=0.3$ in terms of the average $\mathcal{S F R}$ and $s \mathcal{S F R}$ of the $U V J-S F$ sample. No clear trend is found, however, for the fraction of SFGs. 
(vi) We do not find an obvious relationship between SF activity in clusters and the presence of a CC or a BCG forming stars actively.

Our results evidence the impact of the cluster environment on the evolution of its inhabitants and favour a dominant role of physical processes quenching galaxies slowly. The mechanism typically invoked in these cases is strangulation. This process appears to be responsible for the shift of the average $\mathcal{S F R} / s \mathcal{S F R}$ exhibited by SFGs in high-density environments since $z \sim 0.9$, which is interpreted as the evidence of the existence of a large population of transition galaxies below the MS, on their way to be quenched. However, we cannot rule out the impact of other processes occurring at shorter time-scales, such as RPS, which could be partially responsible for a fraction of the SFGs missing in this clusters.

We release the multiwavelength photometry, photometric redshifts, and physical properties of the star-forming cluster members associated to this paper through the RAINBOW Cosmological Database.

\section{ACKNOWLEDGEMENTS}

The authors thank Françoise Combes, Carlos López-Sanjuan, Dieter Lutz, Bianca Poggianti, and Alvio Renzini for their suggestions to improve this work. We acknowledge funding from the INAF PRINSKA 2017 program 1.05.01.88.04. LR-M acknowledges funding support from the Università degli studi di Padova - Dipartimento di Fisica e Astronomia 'G. Galilei'. GR and CM acknowledge support from an INAF PRIN-SKA 2017 grant. PGP-G acknowledges funding support from the Spanish Government MINECO under grants AYA2015-70815-ERC and AYA2015-63650-P. ACE acknowledges support from STFC grant ST/P00541/1. AM acknowledges funding from the INAF PRIN-SKA 2017 program 1.05.01.88.04. Analyses were performed in R 3.4.0 (R Core Team 2018).

\section{REFERENCES}

Abadi M. G., Moore B., Bower R. G., 1999, MNRAS, 308, 947

Abazajian K. N. et al., 2009, ApJS, 182, 543

Abell G. O., 1958, ApJS, 3, 211

Abell G. O., Corwin H. G., Jr., Olowin R. P., 1989, ApJS, 70, 1

Alberts S. et al., 2013, MNRAS, 431, 194

Alberts S. et al., 2014, MNRAS, 437, 437

Alberts S. et al., 2016, ApJ, 825, 72

Annunziatella M. et al., 2014, A\&A, 571, A80

Annunziatella M. et al., 2016, A\&A, 585, A160

Ashby M. L. N. et al., 2013, ApJS, 209, 22

Ashby M. L. N. et al., 2015, ApJS, 218, 33

Bai L., Rieke G. H., Rieke M. J., Christlein D., Zabludoff A. I., 2009, ApJ, 693, 1840

Baldry I. K., Balogh M. L., Bower R. G., Glazebrook K., Nichol R. C., Bamford S. P., Budavari T., 2006, MNRAS, 373, 469

Baldry I. K., Glazebrook K., Brinkmann J., Ivezić Ž., Lupton R. H., Nichol R. C., Szalay A. S., 2004, ApJ, 600, 681

Baldry I. K. et al., 2012, MNRAS, 421, 621

Balestra I. et al., 2016, ApJS, 224, 33

Balogh M. L., Christlein D., Zabludoff A. I., Zaritsky D., 2001, ApJ, 557, 117

Balogh M. L. et al., 2016, MNRAS, 456, 4364

Barger A. J., Cowie L. L., Wang W.-H., 2008, ApJ, 689, 687

Barro G. et al., 2011a, ApJS, 193, 13

Barro G. et al., 2011b, ApJS, 193, 30

Bell E. F. et al., 2004, ApJ, 608, 752
Bell E. F. et al., 2005, ApJ, 625, 23

Berrier J. C., Stewart K. R., Bullock J. S., Purcell C. W., Barton E. J., Wechsler R. H., 2009, ApJ, 690, 1292

Bertin E., Arnouts S., 1996, A\&AS, 117, 393

Biviano A., Fadda D., Durret F., Edwards L. O. V., Marleau F., 2011, A\&A, 532, A77

Biviano A. et al., 2013, A\&A, 558, A1

Blanton M. R., Roweis S., 2007, AJ, 133, 734

Boselli A., Gavazzi G., 2006, PASP, 118, 517

Boselli A. et al., 2016, A\&A, 596, A11

Brammer G. B., van Dokkum P. G., Coppi P., 2008, ApJ, 686, 1503

Brammer G. B. et al., 2011, ApJ, 739, 24

Brodwin M. et al., 2011, ApJ, 732, 33

Brodwin M. et al., 2013, ApJ, 779, 138

Brunner R. J., Lubin L. M., 2000, AJ, 120, 2851

Butcher H., Oemler A., Jr., 1978, ApJ, 219, 18

Butcher H., Oemler A., Jr., 1984, ApJ, 285, 426

Calzetti D., 1997, AJ, 113, 162

Calzetti D., Armus L., Bohlin R. C., Kinney A. L., Koornneef J., StorchiBergmann T., 2000, ApJ, 533, 682

Calzetti D., Kinney A. L., Storchi-Bergmann T., 1994, ApJ, 429, 582

Capak P. et al., 2004, AJ, 127, 180

Cava A. et al., 2009, A\&A, 495, 707

Chabrier G., 2003, PASP, 115, 763

Chary R., Elbaz D., 2001, ApJ, 556, 562

Cimatti A. et al., 2008, A\&A, 482, 21

Coe D. et al., 2012, ApJ, 757, 22

Coenda V., Martínez H. J., Muriel H., 2018, MNRAS, 473, 5617

Cohen J. G., Kneib J.-P., 2002, ApJ, 573, 524

Connor T. et al., 2017, ApJ, 848, 37

Conroy C., Gunn J. E., White M., 2009, ApJ, 699, 486

Cooke E. A. et al., 2016, ApJ, 816, 83

Cooper M. C. et al., 2011, ApJS, 193, 14

Coppin K. E. K. et al., 2011, MNRAS, 416, 680

Cowie L. L., Barger A. J., Hu E. M., Capak P., Songaila A., 2004, AJ, 127, 3137

Dale D. A., Helou G., 2002, ApJ, 576, 159

Darvish B., Mobasher B., Martin D. C., Sobral D., Scoville N., Stroe A., Hemmati S., Kartaltepe J., 2017, ApJ, 837, 16

Darvish B., Mobasher B., Sobral D., Rettura A., Scoville N., Faisst A., Capak P., 2016, ApJ, 825, 113

De Lucia G. et al., 2007, MNRAS, 374, 809

Dickinson M., Giavalisco M., GOODS Team, 2003, The Mass of Galaxies at Low and High Redshift. Springer-Verlag, 324

Domínguez Sánchez H. et al., 2016, MNRAS, 457, 3743

Donahue M. et al., 2015, ApJ, 805, 177

Donahue M. et al., 2016, ApJ, 819, 36

Draine B. T., Li A., 2007, ApJ, 657, 810

Dressler A., 1980, ApJ, 236, 351

Dressler A., Rigby J., Oemler A., Jr., Fritz J., Poggianti B. M., Rieke G., Bai L., 2009, ApJ, 693, 140

Dressler A. et al., 1997, ApJ, 490, 577

Drory N. et al., 2009, ApJ, 707, 1595

Duc P.-A. et al., 2002, A\&A, 382, 60

Ebeling H., Barrett E., Donovan D., Ma C.-J., Edge A. C., van Speybroeck L., 2007, ApJ, 661, L33

Ebeling H., Edge A. C., Henry J. P., 2001, ApJ, 553, 668

Ebeling H., Edge A. C., Mantz A., Barrett E., Henry J. P., Ma C. J., van Speybroeck L., 2010, MNRAS, 407, 83

Ebeling H., Ma C.-J., Barrett E., 2014, ApJS, 211, 21

Egami E. et al., 2010, A\&A, 518, L12

Eisenhardt P. R. M. et al., 2008, ApJ, 684, 905

Elbaz D. et al., 2011, A\&A, 533, A119

Evrard A. E., Metzler C. A., Navarro J. F., 1996, ApJ, 469, 494

Fabian A. C., 1994, ARA\&A, 32, 277

Fadda D., Elbaz D., Duc P.-A., Flores H., Franceschini A., Cesarsky C. J., Moorwood A. F. M., 2000, A\&A, 361, 827

Fazio G. G. et al., 2004, ApJS, 154, 10 
Fernández-Soto A., Lanzetta K. M., Chen H.-W., Levine B., Yahata N., 2002, MNRAS, 330, 889

Finn R. A. et al., 2010, ApJ, 720, 87

Fioc M., Rocca-Volmerange B., 1997, A\&A, 326, 950

Fogarty K., Postman M. , Connor T., Donahue M., Moustakas J., 2015, ApJ, 813, 117

Fogarty K., Postman M., Larson R., Donahue M., Moustakas J., 2017, ApJ, 846,103

Geach J. E. et al., 2006, ApJ, 649, 661

Geller M. J., Hwang H. S., Diaferio A., Kurtz M. J., Coe D., Rines K. J., 2014, ApJ, 783, 52

Gerke B. F. et al., 2007, MNRAS, 376, 1425

Giavalisco M. et al., 2004, ApJ, 600, L93

Girardi M. et al., 2015, A\&A, 579, A4

Grazian A. et al., 2006, A\&A, 449, 951

Griffin M. J. et al., 2010, A\&A, 518, L3

Grillo C. et al., 2015, ApJ, 800, 38

Grogin N. A. et al., 2011, ApJS, 197, 35

Guglielmo V., Poggianti B. M., Moretti A., Fritz J., Calvi R., Vulcani B., Fasano G., Paccagnella A., 2015, MNRAS, 450, 2749

Gunn J. E., Gott J. R., III, 1972, ApJ, 176, 1

Guo Y. et al., 2013, ApJS, 207, 24

Gómez P. L. et al., 2012, AJ, 144, 79

Haines C. P., Gargiulo A., La Barbera F., Mercurio A., Merluzzi P., Busarello G., 2007, MNRAS, 381, 7

Haines C. P. et al., 2009, ApJ, 704, 126

Haines C. P. et al., 2013, ApJ, 775, 126

Haines C. P. et al., 2015, ApJ, 806, 101

Haines C. P. et al., 2017, A\&A, 605, A4

Hoaglin D. C., Mosteller F., Tukey J. W., 1983, Wiley Series in Probability and Mathematical Statistics. Wiley

Huang J.-S. et al., 2004, ApJS, 154, 44

Huchra J. P. et al., 2012, ApJS, 199, 26

Ilbert O. et al., 2009, ApJ, 690, 1236

Jablonka P., Combes F., Rines K., Finn R., Welch T., 2013, A\&A, 557, A103

Jouvel S. et al., 2014, A\&A, 562, A86

Kajisawa M. et al., 2009, ApJ, 702, 1393

Karman W. et al., 2015, A\&A, 574, A11

Kauffmann G., 1995, MNRAS, 274, 153

Kauffmann G. et al., 2003, MNRAS, 341, 33

Kawinwanichakij L. et al., 2017, ApJ, 847, 134

Kennicutt R. C., Evans N. J., 2012, ARA\&A, 50, 531

Kennicutt R. C., Jr., 1998, ARA\&A, 36, 189

Kimm T. et al., 2009, MNRAS, 394, 1131

Kocevski D. D. et al., 2011, ApJ, 736, 38

Koekemoer A. M. et al., 2011, ApJS, 197, 36

Larson R. B., Tinsley B. M., Caldwell C. N., 1980, ApJ, 237, 692

Le Fèvre O. et al., 2003, in Iye M., Moorwood A. F. M., eds, Proc. SPIE Conf. Ser. Vol. 4841, Instrument Design and Performance for Optical/Infrared Ground-based Telescopes. SPIE, Bellingham, p. 1670

Le Fèvre O. et al., 2004, A\&A, 428, 1043

Le Fèvre O. et al., 2015, A\&A, 576, A79

Lewis I. et al., 2002, MNRAS, 334, 673

Lin L. et al., 2014, ApJ, 782, 33

Lotz J. M. et al., 2013, ApJ, 773, 154

Lotz J. M. et al., 2017, ApJ, 837, 97

Lupton R., Blanton M. R., Fekete G., Hogg D. W., O’Mullane W., Szalay A., Wherry N., 2004, PASP, 116, 133

Lutz D. et al., 2011, A\&A, 532, A90

Madau P., Dickinson M., 2014, ARA\&A, 52, 415

Magnelli B. et al., 2013, A\&A, 553, A132

Maier C. et al., 2016, A\&A, 590, A108

Mann A. W., Ebeling H., 2012, MNRAS, 420, 2120

Marcillac D., Rigby J. R., Rieke G. H., Kelly D. M., 2007, ApJ, 654, 825

Martis N. S. et al., 2016, ApJ, 827, L25

McGee S. L., Bower R. G., Balogh M. L., 2014, MNRAS, 442, L105

Mercurio A., Girardi M., Boschin W., Merluzzi P., Busarello G., 2003, A\&A, 397,431
Meurer G. R., Heckman T. M., Calzetti D., 1999, ApJ, 521, 64

Mignoli M. et al., 2005, A\&A, 437, 883

Molino A. et al., 2017, MNRAS, 470, 95

Moore B., Katz N., Lake G., Dressler A., Oemler A., 1996, Nature, 379, 613

Mortlock A. et al., 2015, MNRAS, 447, 2

Moutard T., Sawicki M., Arnouts S., Golob A., Malavasi N., Adami C., Coupon J., Ilbert O., 2018, MNRAS, 479, 2147

Muzzin A. et al., 2014, ApJ, 796, 65

Nantais J. B. et al., 2016, A\&A, 592, A161

Nantais J. B. et al., 2017, MNRAS, 465, L104

Nayyeri H. et al., 2017, ApJS, 228, 7

Newman A. B., Treu T., Ellis R. S., Sand D. J., Nipoti C., Richard J., Jullo E., 2013, ApJ, 765, 24

Noeske K. G. et al., 2007, ApJ, 660, L43

Ogrean G. A. et al., 2015, ApJ, 812, 153

Oliver S. J. et al., 2012, MNRAS, 424, 1614

Paccagnella A. et al., 2016, ApJ, 816, L25

Paccagnella A. et al., 2017, ApJ, 838, 148

Pacifici C., Charlot S., Blaizot J., Brinchmann J., 2012, MNRAS, 421, 2002

Papovich C. et al., 2018, ApJ, 854, 30

Patel S. G., Holden B. P., Kelson D. D., Illingworth G. D., Franx M., 2009, ApJ, 705, L67

Pelló R. et al., 2009, A\&A, 508, 1173

Peng Y.-j. et al., 2010, ApJ, 721, 193

Pilbratt G. L. et al., 2010, A\&A, 518, L1

Poggianti B. M., 2003, Ap\&SS, 285, 121

Poggianti B. M., Bridges T. J., Komiyama Y., Yagi M., Carter D., Mobasher B., Okamura S., Kashikawa N., 2004, ApJ, 601, 197

Poggianti B. M., Smail I., Dressler A., Couch W. J., Barger A. J., Butcher H., Ellis R. S., Oemler A., Jr, 1999, ApJ, 518, 576

Poggianti B. M. et al., 2009, ApJ, 693, 112

Poggianti B. M. et al., 2016, AJ, 151, 78

Poggianti B. M. et al., 2017, ApJ, 844, 48

Poglitsch A. et al., 2010, A\&A, 518, L2

Polletta M. et al., 2007, ApJ, 663, 81

Popesso P. et al., 2011, A\&A, 532, A145

Popping G., Puglisi A., Norman C. A., 2017, MNRAS, 472, 2315

Postman M. et al., 2012, ApJS, 199, 25

Pérez-González P. G. et al., 2005, ApJ, 630, 82

Pérez-González P. G. et al., 2008, ApJ, 675, 234

Pérez-González P. G. et al., 2010, A\&A, 518, L15

Pérez-González P. G. et al., 2013, ApJ, 762, 46

Quadri R. F., Williams R. J., Franx M., Hildebrandt H., 2012, ApJ, 744, 88

Ravindranath S., Ho L. C., 2002, ApJ, 577, 133

Rawle T. D. et al., 2010, A\&A, 518, L14

Rawle T. D. et al., 2012a, ApJ, 747, 29

Rawle T. D. et al., 2012b, ApJ, 756, 106

Rawle T. D. et al., 2014, MNRAS, 442, 196

Rawle T. D. et al., 2016, MNRAS, 459, 1626

R Core Team, 2018, in R: A Language and Environment for Statistical Computing. R Foundation for Statistical Computing, Vienna, Austria. Available at: https://www.R-project.org/

Renzini A., Peng Y.-j., 2015, ApJ, 801, L29

Rex M. et al., 2010, A\&A, 518, L13

Rieke G. H., Alonso-Herrero A., Weiner B. J., Pérez-González P. G., Blaylock M., Donley J. L., Marcillac D., 2009, ApJ, 692, 556

Rieke G. H. et al., 2004, ApJS, 154, 25

Rodighiero G. et al., 2011, ApJ, 739, L40

Rosati P. et al., 2014, The Messenger, 158, 48

Rumsey C. et al., 2016, MNRAS, 460, 569

Saintonge A., Tran K.-V. H., Holden B. P., 2008, ApJ, 685, L113

Salpeter E. E., 1955, ApJ, 121, 161

Sanders D. B. et al., 2007, ApJS, 172, 86

Santos J. S., Rosati P., Tozzi P., Böhringer H., Ettori S., Bignamini A., 2008, A\&A, 483, 35

Santos J. S. et al., 2015, MNRAS, 447, L65 
Sargent M. T., Béthermin M., Daddi E., Elbaz D., 2012, ApJ, 747, L31

Schechter P., 1976, ApJ, 203, 297

Schmidt K. B. et al., 2014, ApJ, 782, L36

Schreiber C., Pannella M., Leiton R., Elbaz D., Wang T., Okumura K., Labbé I., 2017, A\&A, 599, A134

Scoville N. et al., 2007, ApJS, 172, 1

Shectman S. A., Landy S. D., Oemler A., Tucker D. L., Lin H., Kirshner R. P., Schechter P. L., 1996, ApJ, 470, 172

Sheth R. K., Rossi G., 2010, MNRAS, 403, 2137

Smith R. J. et al., 2010, MNRAS, 408, 1417

Springel V. et al., 2005, Nature, 435, 629

Steinhauser D., Schindler S., Springel V., 2016, A\&A, 591, A51

Stroe A. et al., 2015, MNRAS, 450, 646

Sunyaev R. A., Zel'dovich Y. B., 1972, Comments Astrophys. Space Phys., 4,173

Szokoly G. P. et al., 2004, ApJS, 155, 271

Tran K.-V. H., Franx M., Illingworth G. D., van Dokkum P., Kelson D. D., Blakeslee J. P., Postman M., 2007, ApJ, 661, 750

Treu T. et al., 2015, ApJ, 812, 114

Tyler K. D., Rieke G. H., Bai L., 2013, ApJ, 773, 86

Umetsu K. et al., 2014, ApJ, 795, 163

van den Bosch F. C., Aquino D., Yang X., Mo H. J., Pasquali A., McIntosh D. H., Weinmann S. M., Kang X., 2008, MNRAS, 387, 79

van der Burg R. F. J., McGee S., Aussel H., Dahle H., Arnaud M., Pratt G. W., Muzzin A., 2018, A\&A, 618, A140

van der Burg R. F. J. et al., 2013, A\&A, 557, A15

Vanzella E. et al., 2008, A\&A, 478, 83

Vulcani B., Poggianti B. M., Finn R. A., Rudnick G., Desai V., Bamford S., 2010, ApJ, 710, L1

Vulcani B. et al., 2011, MNRAS, 412, 246

Vulcani B. et al., 2012, MNRAS, 420, 1481

Vulcani B. et al., 2013, A\&A, 550, A58

Vulcani B. et al., 2016, ApJ, 833, 178

Vulcani B. et al., 2017, ApJ, 837, 126

Wang W.-H., Cowie L. L., Barger A. J., Keenan R. C., Ting H.-C., 2010, ApJS, 187, 251

Wetzel A. R., Tinker J. L., Conroy C., van den Bosch F. C., 2013, MNRAS, 432,336

Whitaker K. E., Kriek M., van Dokkum P. G., Bezanson R., Brammer G., Franx M., Labbé I., 2012a, ApJ, 745, 179

Whitaker K. E., van Dokkum P. G., Brammer G., Franx M., 2012b, ApJ, 754, L29

Whitaker K. E. et al., 2015, ApJ, 811, L12

Wijesinghe D. B. et al., 2012, MNRAS, 423, 3679

Williams R. J., Quadri R. F., Franx M., van Dokkum P., Labbé I., 2009, ApJ, 691,1879

Wirth G. D. et al., 2004, AJ, 127, 3121

Wittman D., Bhaskar R., Tobin R., 2016, MNRAS, 457, 4005

Wuyts S., Labbé I., Förster Schreiber N. M., Franx M., Rudnick G., Brammer

G. B., van Dokkum P. G., 2008, ApJ, 682, 985

Wuyts S. et al., 2007, ApJ, 655, 51

Zitrin A. et al., 2013, ApJ, 762, L30

Zitrin A. et al., 2015, ApJ, 801, 44

\section{SUPPORTING INFORMATION}

Supplementary data are available at MNRAS online.

Figure 7. Thumbnails of the cluster members from the M-FIR sample ordered by increasing redshift.

Please note: Oxford University Press is not responsible for the content or functionality of any supporting materials supplied by the authors. Any queries (other than missing material) should be directed to the corresponding author for the article.

\section{APPENDIX A: DATA AVAILABLE ON THE CANDELS FIELDS}

In the following subsections, we briefly enumerate the photometric and spectroscopic data on the CANDELS fields which are used in our analysis.

\section{A1 GOODS-S}

We use the multiwavelength catalogue on the CANDELS/GOODSS field published by Guo et al. (2013), which combines the CANDELS HST/WFC3 F105W, F125W, and F160W bands with data from UV ( $U$ band from both CTIO/MOSAIC and VLT/VIMOS), optical (HST/ACS F435W, F606W, F775W, F814W, and F850LP), and IR (HST/WFC3 F098M, VLT/ISAAC $K s$, VLT/HAWK-I $K s$, and Spitzer/IRAC 3.6, 4.5, 5.8, and $8.0 \mu \mathrm{m}$ ) observations. The catalogue is based on source detection in the WFC3 F160W band. Applying the methodology described in Section 3, we complement the catalogue with MIR photometry in Spitzer/MIPS 24 and $70 \mu \mathrm{m}$ from Pérez-González et al. (2008), and FIR photometry from the GOODS-Herschel (Elbaz et al. 2011) and the PACS Evolutionary Probe (PEP; Magnelli et al. 2013) surveys, including PACS 100 and $160 \mu \mathrm{m}$, and SPIRE 250, 350, and $500 \mu \mathrm{m}$. The spectroscopic data are gathered from the VIMOS VLT Deep Survey (Le Fèvre et al. 2004; Szokoly et al. 2004), the K20 survey (Mignoli et al. 2005), and other surveys such as those carried out by e.g. Cimatti et al. (2008) and Vanzella et al. (2008). See Guo et al. (2013) for the details.

\section{A2 GOODS-N}

The multiwavelength catalogue used on CANDELS/GOODS-N is built and described by Barro et al. (in preparation) and includes UV to FIR and radio data. In particular, UV data from GALEX (PI: C. Martin), ground-based optical data from $U$ to $z$ bands taken with the Mosaic camera mounted on the Kitt Peak telescope and with the Subaru/Suprime-Cam as part of the Hawaii Hubble Deep Field North project (Capak et al. 2004); 25 medium bands from the GTC Survey for High-z Absorption Red and Dead Sources (Pérez-González et al. 2013) survey; $J, H$, and $K s$ imaging from the Subaru/MOIRCS deep survey (Kajisawa et al. 2009) and CFHT/WIRCam $K s$ photometry (Lin, in preparation); Spitzer/IRAC 3.6, 4.5, 5.8, and $8.0 \mu \mathrm{m}$ maps from Spitzer GOODS (Dickinson et al. 2003), SEDS (Ashby et al. 2013), and SCANDELS (Ashby et al. 2015); Spitzer/MIPS data from the GOODS Far-Infrared Deep Extragalactic Legacy survey (PI: M. Dickinson); Herschel from the GOODS-Herschel (Elbaz et al. 2011) and PEP (Magnelli et al. 2013) surveys, including PACS 100 and $160 \mu \mathrm{m}$, and SPIRE 250, 350 , and $500 \mu \mathrm{m}$. The spectroscopic redshifts used are a compilation based primarily on ACS-GOODS redshift survey (Cowie et al. 2004; Barger, Cowie \& Wang 2008), the Team Keck Redshift Survey (Wirth et al. 2004), and the DEEP3 galaxy redshift survey (Cooper et al. 2011).

\section{A3 COSMOS}

We use the multiwavelength catalogue on the CANDELS/COSMOS field published by Nayyeri et al. (2017), which combines the CANDELS HST/WFC3 F105W, F125W, and F160W bands with data from HST/ACS F606W and F814W, CFHT/MegaPrime in the 

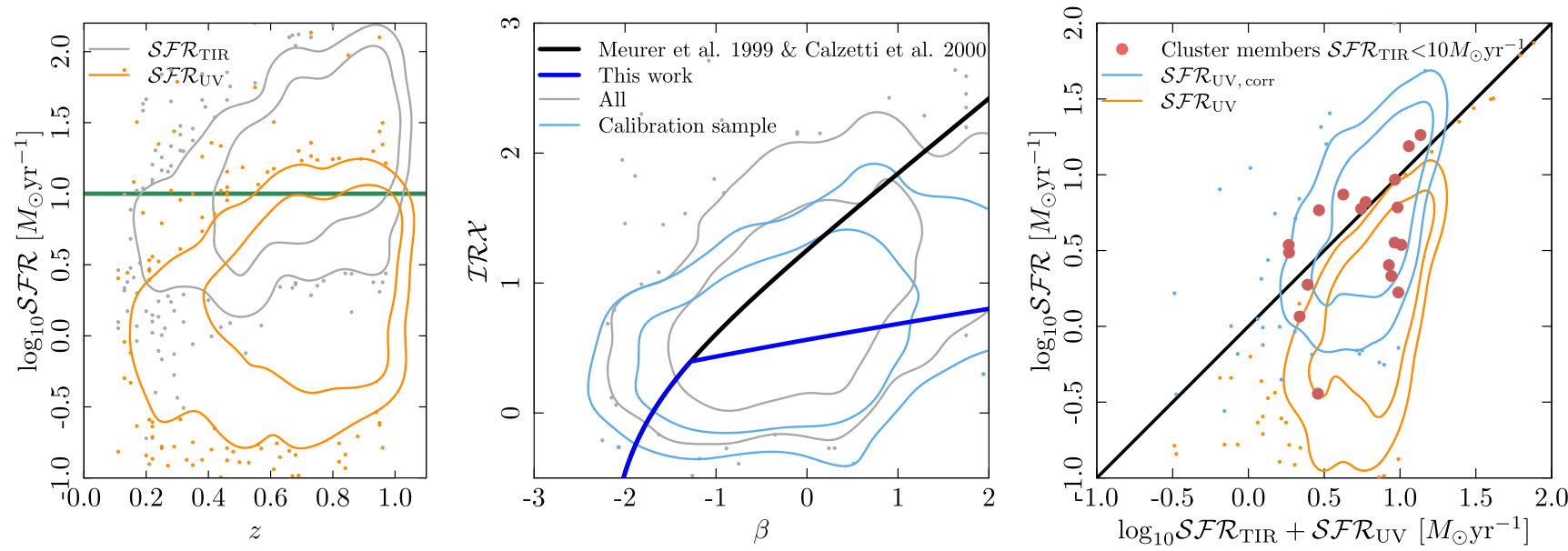

Figure A1 Left-hand panel: $\mathcal{S} \mathcal{F} \mathcal{R}_{\mathrm{TIR}}$ (grey contours) and $\mathcal{S F R}_{\mathrm{UV}}$ (orange contours) versus redshift for all the 1548 MIR/FIR-detected galaxies in CANDELS fields with $U V J$ colours corresponding to SFGs, $\log _{10} \mathcal{M}_{*} / M_{\odot}>10$, and $0.1<z<1.0$. The green line represents the selection criteria for the selection of the calibration sample. Central panel: $\mathcal{I R} \mathcal{X}-\beta$ relation for the galaxies in CANDELS fields with $U V J$ colours corresponding to $\mathrm{SFGs}, \log _{10} \mathcal{M}_{*} / M_{\odot}>10$, and $0.1<z<1.0$ (grey contours), and the 525 galaxies with $\mathcal{S F \mathcal { R }}$ TIR $<10 M_{\odot} \mathrm{yr}^{-1}$ of which the calibration sample is made of (blue contours). We represent our calibration with a blue line. The black line is the $\mathcal{I R} \mathcal{X}-\beta$ fit from Meurer et al. (1999) modified with a Calzetti et al. (2000) extinction law to the UV wavelength we consider in our study (2800 ̊). Right-hand panel: comparison between $\mathcal{S F R} \mathcal{R}_{\mathrm{TIR}}+\mathcal{S F} \mathcal{R}_{\mathrm{UV}}$ and the $\mathcal{S} \mathcal{F} \mathcal{R}_{\mathrm{UV}}$,corr. corrected for dust extinction using our own calibration (equation B1, blue contours). For comparison, we show the distribution of values of $\mathcal{S F R} \mathcal{R}_{U V}$ previous to the dust extinction correction (orange contours). To evaluate the behaviour of our UV correction in the clusters, we represent the comparison between the $\mathcal{S F} \mathcal{R}_{\mathrm{TOT}}$ and the $\mathcal{S} \mathcal{F} \mathcal{R}_{\mathrm{UV}}$,corr. of the cluster members with $\mathcal{S} \mathcal{F} \mathcal{R}_{\mathrm{TIR}}<10 M_{\odot} \mathrm{yr}^{-1}$.

$u *, g *, r *, i *$, and $z *$ bands, from the Subaru/Suprime-Cam in the $B, g^{+}, V, r^{+}, i^{+}$, and $z^{+}$, along with 12 intermediate and two narrow bands ( $\sim 4000-8500 \AA)$, from the VLT/VISTA in the $Y, J$, $H$, and $K s$ bands, Mayall/NEWFIRM $J_{1}, J_{2}, J_{3}, H_{1}, H_{2}, K$, and Spitzer/IRAC 3.6, 4.5, 5.8, and $8.0 \mu \mathrm{m}$ bands. Again, we combine this catalogue with MIR photometry in Spitzer/MIPS 24 and $70 \mu \mathrm{m}$ from Sanders et al. (2007), and FIR photometry including PACS 100 and $160 \mu \mathrm{m}$ from PEP program (Lutz et al. 2011), and SPIRE 250, 350, and $500 \mu \mathrm{m}$ from the Herschel Multi-tiered Extragalactic Survey (Oliver et al. 2012). Among the spectroscopic surveys gathered, we highlight the VIMOS Ultra Deep Survey (Le Fèvre et al. 2015) and zCOSMOS (PI: S. Lilly).

\section{APPENDIX B: UV CORRECTION}

The ratio of the $\mathcal{L}_{\text {TIR }}$ to $\mathcal{L}_{\mathrm{UV}}$, usually referred as $\mathcal{I R} \mathcal{X}$, is tightly related to the dust attenuation in a galaxy. This is because dust absorbs and scatters mainly UV photons obscuring and reddening the galaxy SED at wavelengths $\lesssim 1 \mu \mathrm{m}$. Then, it re-emits the absorbed energy in the IR, at wavelengths $\sim 1-1000 \mu \mathrm{m}$. Since the work of Meurer et al. (1999) on local starburst galaxies (i.e. extreme SFGs), the relation between the $\mathcal{I R} \mathcal{X}$ and the slope of the UV $(\beta)$ has been frequently used to estimate the UV dust attenuation of galaxies. In practice, this relation is calibrated for local blue galaxies for which FIR observations is available (e.g. Calzetti 1997, Meurer et al. 1999) and then, it is used to correct the UV luminosity from extinction up to high redshifts (Meurer et al. 1999). However, important deviations from these relations have been observed, for example for galaxies forming stars at a lower rates or at different redshifts. Lately, different studies have explored in detailed the physical origin of variations in the $\mathcal{I} \mathcal{X}-\beta$ relation (e.g. Popping, Puglisi \& Norman 2017). In this context, we aim at deriving an optimized dust attenuation correction (i.e. $\mathcal{I} \mathcal{R} \mathcal{X}-\beta$ relation) that we can apply to those star-forming cluster members fainter than our observational limits in MIPS and/or Herschel, and therefore presumably less star-forming than the starbursts on which the calibrations in the literature are defined.

Following a similar approach to Domínguez Sánchez et al. (2016), we basically derive a $\mathcal{I} \mathcal{R} \mathcal{X}-\beta$ relation for a sample of SFGs which are faint MIR/FIR emitters. In particular, we take advantage of the deep coverage on CANDELS fields (GOODS and COSMOS) to select a subsample of SFGs fainter than the CLASH+HLS fields observational limits in MIPS and/or Herschel bands. We only consider galaxies classified as SFGs using an UVJ diagram, located in the redshift range between 0.1 and 1.0, and with $\log _{10} \mathcal{M}_{*} / M_{\odot}>10$. In Fig. A1 (left-hand panel), we display the distribution with redshift of $\mathcal{S F} \mathcal{R}_{\mathrm{TIR}}$ and $\mathcal{S} \mathcal{F} \mathcal{R}_{\mathrm{UV}}$ of these galaxies [obtained following equations (5) and (6), respectively]. The calibration sample includes the 1548 galaxies with $\mathcal{S} \mathcal{F} \mathcal{R}_{\mathrm{TIR}}<$ $10 M_{\odot} \mathrm{yr}^{-1}$ (green horizontal line).

Once the sample is defined, we compute the UV slope for each galaxy using a linear interpolation between 1500 and $2800 \AA$ in the best-fitting templates given by RAINBOW (Section 5). The typical uncertainty in the $\beta$ values is $\sim 20$ per cent. Then, we compute their $\mathcal{I R} \mathcal{X}$ as the ratio of their $\mathcal{S F R}$ TIR and $\mathcal{S F} \mathcal{R}_{\mathrm{UV}}$. In Fig. A1 (central panel), we display the $\mathcal{I} \mathcal{R} \mathcal{X}-\beta$ space for the whole field sample of MIR/FIR emitters $\left(\log _{10} \mathcal{M}_{*} / M_{\odot}>10\right.$ and $0.1<z<1.0$; grey contours), and the calibration sample of faint MIR/FIR emitters (blue contours). Then, we fit the points in the $\mathcal{I} \mathcal{R} \mathcal{X}-\beta$ plane for our calibration sample with a linear function. We derive the following best-fitting expression:

$\mathcal{A}_{\mathrm{UV}}=(1.76 \pm 0.04)+(0.20 \pm 0.02) \beta$

Again, following the approach by Domínguez Sánchez et al. (2016), we apply the Meurer et al. (1999) $\mathcal{I} \mathcal{R} \mathcal{X}-\beta$ relation $\left(\mathcal{A}_{1600}=4.43+\right.$ $1.99 \beta$ ) for $\beta$ values lower than the point in which our fit intercepts the relation by Meurer et al. (1999), $\beta=-1.7$, and equation (B1) for higher $\beta$ values. 
To assess the efficiency of our calibration, we quantify the scatter of the difference between the $\mathcal{S} \mathcal{F} \mathcal{R}_{\text {TOT }}$ derived as the addition of $\mathcal{S F} \mathcal{R}_{\mathrm{TIR}}$ and $\mathcal{S} \mathcal{F} \mathcal{R}_{\mathrm{UV}}$, and the $\mathcal{S} \mathcal{F} \mathcal{R}_{\mathrm{TOT}}$ computed as the $\mathcal{S} \mathcal{F} \mathcal{R}_{\mathrm{UV}}$ corrected for dust extinction for our calibration sample (right-hand panel in Fig. A1). The values vary between -0.38 and 0.26 dex with a median of -0.02 dex. Using the calibration by Meurer et al. (1999) instead would have led to a median absolute deviation of 0.53 dex. Given that we use the calibration built on field galaxies to correct also the $\mathcal{S} \mathcal{F} \mathcal{R}_{\mathrm{UV}}$ of the cluster members not detected in the MIR/FIR, we compare how the calibration behaves for those faint
MIR/FIR cluster members $\left(\mathcal{S F \mathcal { F }} \mathcal{T I R}<10 M_{\odot} \mathrm{yr}^{-1}\right)$. In the righthand panel of Fig. A1, we see that the dust extinction correction behaves similarly in the field and the clusters. For the latter, the median absolute deviation is -0.05 dex, and the differences vary between -0.54 and 0.23 dex.

\section{APPENDIX C: CATALOGUES}

This appendix details the entries of the catalogues released through the RAINBOW Cosmological Database.

Table C1. Multiwavelength photometry.

\begin{tabular}{ll}
\hline Entry name & \multicolumn{1}{c}{ Description } \\
\hline object & ID of the source in the parent catalogue. This ID is not the CLASH catalogue ID. \\
flux & $(\mu \mathrm{Jy})$ \\
err_flux & $(\mu \mathrm{Jy})$ \\
\hline
\end{tabular}

Table C2. Flags for the MIPS counterpart identification.

\begin{tabular}{|c|c|}
\hline Entry name & Description \\
\hline MIPS_n_counterparts & Total number of (selection band) counterparts candidates for the MIPS $24 \mu \mathrm{m}$ source. \\
\hline & The most probable counterpart is flagged with a ' -1 '. \\
\hline MIPS_order & The order of likelihood of being the right counterpart of the MIPS source. \\
\hline MIPS_discriminator & Quantity used to determine the counterpart likelihood order. \\
\hline MIPS_fIRAC80 & IRAC $8.0 \mu \mathrm{m}$ flux ( $\mu \mathrm{Jy}$ ) used for the MIPS $24 \mu \mathrm{m}$ counterpart identification. \\
\hline MIPS_err_fIRAC80 & IRAC $8.0 \mu \mathrm{m}$ flux error ( $\mu \mathrm{Jy})$ used for the MIPS $24 \mu \mathrm{m}$ counterpart identification. \\
\hline MIPS_fIRAC36 & IRAC $3.6 \mu$ m flux ( $\mu$ Jy) used for the MIPS $24 \mu \mathrm{m}$ counterpart identification. \\
\hline MIPS_err_fIRAC36 & IRAC $3.6 \mu \mathrm{m}$ flux error $(\mu \mathrm{Jy})$ used for the MIPS $24 \mu \mathrm{m}$ counterpart identification. \\
\hline MIPS_distance & Distance between the MIPS $24 \mu \mathrm{m}$ source and the counterpart candidate. \\
\hline MIPS24_snr_cuts & $\begin{array}{l}\text { Flag regarding the S/N cuts applied in MIPS } 24 \mu \mathrm{m} \text { : } \\
0 \text { no-flux, } 1 \text { flux }>\mathrm{S} / \mathrm{N} \text { limit, }-1 \text { flux }<\mathrm{S} / \mathrm{N} \text { limit. }\end{array}$ \\
\hline n_IRAC_MIPS24_psf0.25/0.5/1/2 & Number of IRAC sources within the MIPS $24 \mu \mathrm{m}$ PSF $(\times 0.25 / 0.5 / 1 / 2)$ \\
\hline n_IRAC_MIPS24_wcs0.25/0.5/1/2 & Number of IRAC sources within the MIPS $24 \mu \mathrm{m}$ WCS accuracy $(\times 0.25 / 0.5 / 1 / 2)$. \\
\hline
\end{tabular}


Table C3. Flags for the PACS counterpart identification.

\begin{tabular}{|c|c|}
\hline Entry name & Description \\
\hline PACS_ID_order & $\begin{array}{l}\text { ID of the PACS counterpart flagged with the likelihood. } \\
\text { The most probable counterpart is flaged with a ' }-1 \text { '. }\end{array}$ \\
\hline PACS_discriminator & Quantity used to determine the counterpart likelihood order. \\
\hline PACS_fPACS160 & PACS $160 \mu \mathrm{m}$ flux ( $\mu \mathrm{Jy}$ ) used for the PACS counterpart identification. \\
\hline PACS_err_fPACS160 & PACS $160 \mu \mathrm{m}$ flux error $(\mu \mathrm{Jy})$ used for the PACS counterpart identification. \\
\hline PACS_fMIPS24 & MIPS $24 \mu \mathrm{m}$ flux ( $\mu \mathrm{Jy}$ ) used for the PACS counterpart identification. \\
\hline PACS_err_fMIPS24 & MIPS $24 \mu \mathrm{m}$ flux error ( $\mu \mathrm{Jy}$ ) used for the PACS counterpart identification. \\
\hline PACS_fIRAC80 & IRAC $8.0 \mu \mathrm{m}$ flux ( $\mu \mathrm{Jy}$ ) used for the PACS counterpart identification. \\
\hline PACS_err_fIRAC80 & IRAC $8.0 \mu \mathrm{m}$ flux error ( $\mu \mathrm{Jy})$ used for the PACS counterpart identification. \\
\hline PACS_fIRAC36 & IRAC $3.6 \mu \mathrm{m}$ flux ( $\mu \mathrm{Jy}$ ) used for the PACS counterpart identification. \\
\hline PACS_err_fIRAC36 & IRAC $3.6 \mu \mathrm{m}$ flux error ( $\mu \mathrm{Jy}$ ) used for the PACS counterpart identification. \\
\hline PACS160_snr_cuts & $\begin{array}{l}\text { Flag regarding the } \mathrm{S} / \mathrm{N} \text { cuts applied in PACS } 160 \mu \mathrm{m}: \\
0 \text { no-flux, } 1 \text { flux }>\mathrm{S} / \mathrm{N} \text { limit, }-1 \text { flux }<\mathrm{S} / \mathrm{N} \text { limit. }\end{array}$ \\
\hline n_PACS100_psf0.25/0.5/1/2 & Number of sources in the parent catalogue within the PACS $100 \mu \mathrm{m}$ PSF $(\times 0.25 / 0.5 / 1 / 2)$. \\
\hline n_PACS160_psf0.25/0.5/1/2 & Number of sources in the parent catalogue within the PACS $160 \mu \mathrm{m}$ PSF $(\times 0.25 / 0.5 / 1 / 2)$ \\
\hline n_PACS100_wcs0.25/0.5/1/2 & Number of sources in the parent catalogue within the PACS $100 \mu \mathrm{m}$ WCS accuracy $(\times 0.25 / 0.5 / 1 / 2)$. \\
\hline n_PACS160_wcs $0.25 / 0.5 / 1 / 2$ & Number of sources in the parent catalogue within the PACS $160 \mu \mathrm{m}$ WCS accuracy $(\times 0.25 / 0.5 / 1 / 2)$. \\
\hline n_PACS_PACS100_psf0.25/0.5/1/2 & Number of PACS sources within the PACS $100 \mu \mathrm{m}$ PSF $(\times 0.25 / 0.5 / 1 / 2)$ \\
\hline n_PACS_PACS160_psf0.25/0.5/1/2 & Number of PACS sources within the PACS $160 \mu \mathrm{m}$ PSF $(\times 0.25 / 0.5 / 1 / 2)$ \\
\hline n_PACS_PACS100_wcs0.25/0.5/1/2 & Number of PACS sources within the PACS $100 \mu \mathrm{m}$ WCS accuracy $(\times 0.25 / 0.5 / 1 / 2)$. \\
\hline n_PACS_PACS160_wcs0.25/0.5/1/2 & Number of PACS sources within the PACS $160 \mu \mathrm{m}$ WCS accuracy $(\times 0.25 / 0.5 / 1 / 2)$. \\
\hline n_MIPS_PACS100_psf0.25/0.5/1/2 & Number of MIPS sources within the PACS $100 \mu \mathrm{m}$ PSF $(\times 0.25 / 0.5 / 1 / 2)$ \\
\hline n_MIPS_PACS160_psf0.25/0.5/1/2 & Number of MIPS sources within the PACS $160 \mu \mathrm{m}$ PSF $(\times 0.25 / 0.5 / 1 / 2)$. \\
\hline
\end{tabular}


Table C4. Flags for the SPIRE counterpart identification.

\begin{tabular}{|c|c|}
\hline Entry name & Description \\
\hline object & ID of the source in the parent catalogue. \\
\hline SPIRE_ID_order & $\begin{array}{l}\text { ID of the SPIRE counterpart flagged with the likelihood. } \\
\text { The most probable counterpart is flaged with a ' } 1 \text { '. }\end{array}$ \\
\hline SPIRE_discriminator & Quantity used to determine the counterpart likelihood order. \\
\hline SPIRE_fSPIRE500 & SPIRE $500 \mu \mathrm{m}$ flux ( $\mu \mathrm{Jy}$ ) used for the SPIRE counterpart identification. \\
\hline SPIRE_err_fSPIRE500 & SPIRE $500 \mu \mathrm{m}$ flux error ( $\mu \mathrm{Jy}$ ) used for the SPIRE counterpart identification. \\
\hline SPIRE_fSPIRE350 & SPIRE $350 \mu \mathrm{m}$ flux ( $\mu \mathrm{Jy}$ ) used for the SPIRE counterpart identification. \\
\hline SPIRE_err_fSPIRE350 & SPIRE $350 \mu \mathrm{m}$ flux error ( $\mu \mathrm{Jy}$ ) used for the SPIRE counterpart identification. \\
\hline SPIRE_fSPIRE250 & SPIRE $250 \mu \mathrm{m}$ flux ( $\mu \mathrm{Jy}$ ) used for the SPIRE counterpart identification. \\
\hline SPIRE_err_fSPIRE250 & SPIRE $250 \mu \mathrm{m}$ flux error ( $\mu \mathrm{Jy}$ ) used for the SPIRE counterpart identification. \\
\hline SPIRE_fPACS160 & PACS $160 \mu \mathrm{m}$ flux ( $\mu \mathrm{Jy}$ ) used for the SPIRE counterpart identification. \\
\hline SPIRE_err_fPACS160 & PACS $160 \mu \mathrm{m}$ flux error ( $\mu \mathrm{Jy}$ ) used for the SPIRE counterpart identification. \\
\hline SPIRE_fPACS100 & PACS $100 \mu \mathrm{m}$ flux ( $\mu \mathrm{Jy}$ ) used for the SPIRE counterpart identification. \\
\hline SPIRE_err_fPACS100 & PACS $100 \mu \mathrm{m}$ flux error ( $\mu \mathrm{Jy})$ used for the SPIRE counterpart identification. \\
\hline SPIRE_fMIPS24 & MIPS $24 \mu \mathrm{m}$ flux ( $\mu \mathrm{Jy}$ ) used for the SPIRE counterpart identification. \\
\hline SPIRE_err_fMIPS24 & MIPS $24 \mu \mathrm{m}$ flux error ( $\mu \mathrm{Jy}$ ) used for the SPIRE counterpart identification. \\
\hline SPIRE_fIRAC80 & IRAC $8.0 \mu \mathrm{m}$ flux ( $\mu \mathrm{Jy}$ ) used for the SPIRE counterpart identification. \\
\hline SPIRE_err_fIRAC80 & IRAC $8.0 \mu \mathrm{m}$ flux error ( $\mu \mathrm{Jy})$ used for the SPIRE counterpart identification. \\
\hline SPIRE_fIRAC36 & IRAC $3.6 \mu \mathrm{m}$ flux ( $\mu \mathrm{Jy}$ ) used for the SPIRE counterpart identification. \\
\hline SPIRE_err_fIRAC36 & IRAC $3.6 \mu \mathrm{m}$ flux error ( $\mu \mathrm{Jy})$ used for the SPIRE counterpart identification. \\
\hline SPIRE_distance & Distance between the SPIRE and the counterpart candidate. \\
\hline SPIRE_order & The order of likelihood of being the right counterpart of the SPIRE source. \\
\hline SPIRE_n_counterparts & Total number of counterparts candidates for the SPIRE source. \\
\hline SPIRE250_snr_cuts & $\begin{array}{l}\text { Flag regarding the } \mathrm{S} / \mathrm{N} \text { cuts applied in SPIRE } 250 \mu \mathrm{m} \text { : } \\
0 \text { no-flux, } 1 \text { flux }>\mathrm{S} / \mathrm{N} \text { limit, }-1 \text { flux }<\mathrm{S} / \mathrm{N} \text { limit. }\end{array}$ \\
\hline SPIRE350_snr_cuts & $\begin{array}{l}\text { Flag regarding the } \mathrm{S} / \mathrm{N} \text { cuts applied in SPIRE } 350 \mu \mathrm{m} \text { : } \\
0 \text { no-flux, } 1 \text { flux }>\mathrm{S} / \mathrm{N} \text { limit, }-1 \text { flux }<\mathrm{S} / \mathrm{N} \text { limit. }\end{array}$ \\
\hline SPIRE500_snr_cuts & $\begin{array}{l}\text { Flag regarding the } \mathrm{S} / \mathrm{N} \text { cuts applied in SPIRE } 500 \mu \mathrm{m} \text { : } \\
0 \text { no-flux, } 1 \text { flux }>\mathrm{S} / \mathrm{N} \text { limit, }-1 \text { flux }<\mathrm{S} / \mathrm{N} \text { limit. }\end{array}$ \\
\hline n_SPIRE250_psf0.25/0.5/1/2 & Number of sources in the parent catalogue within the SPIRE $250 \mu \mathrm{m}$ PSF $(\times 0.25 / 0.5 / 1 / 2)$. \\
\hline n_SPIRE350_psf0.25/0.5/1/2 & Number of sources in the parent catalogue within the SPIRE $350 \mu \mathrm{m}$ PSF $(\times 0.25 / 0.5 / 1 / 2)$. \\
\hline n_SPIRE500_psf0.25/0.5/1/2 & Number of sources in the parent catalogue within the SPIRE $500 \mu \mathrm{m}$ PSF $(\times 0.25 / 0.5 / 1 / 2)$. \\
\hline n_SPIRE250_wcs0.25/0.5/1/2 & Number of sources in the parent catalogue within the SPIRE $250 \mu \mathrm{m}$ WCS accuracy $(\times 0.25 / 0.5 / 1 / 2)$. \\
\hline n_SPIRE350_wcs $0.25 / 0.5 / 1 / 2$ & Number of sources in the parent catalogue within the SPIRE $350 \mu \mathrm{m}$ WCS accuracy $(\times 0.25 / 0.5 / 1 / 2)$. \\
\hline n_SPIRE500_wcs0.25/0.5/1/2 & Number of sources in the parent catalogue within the SPIRE $500 \mu \mathrm{m}$ WCS accuracy $(\times 0.25 / 0.5 / 1 / 2)$. \\
\hline n_SPIRE_SPIRE250_psf0.25/0.5/1/2 & Number of SPIRE sources within the SPIRE $250 \mu \mathrm{m}$ PSF $(\times 0.25 / 0.5 / 1 / 2)$ \\
\hline n_SPIRE_SPIRE350_psf0.25/0.5/1/2 & Number of SPIRE sources within the SPIRE $350 \mu \mathrm{m}$ PSF $(\times 0.25 / 0.5 / 1 / 2)$. \\
\hline n_SPIRE_SPIRE500_psf0.25/0.5/1/2 & Number of SPIRE sources within the SPIRE $500 \mu \mathrm{m}$ PSF $(\times 0.25 / 0.5 / 1 / 2)$. \\
\hline n_SPIRE_SPIRE250_wcs $0.25 / 0.5 / 1 / 2$ & Number of SPIRE sources within the SPIRE $250 \mu \mathrm{m}$ WCS accuracy $(\times 0.25 / 0.5 / 1 / 2)$. \\
\hline n_SPIRE_SPIRE350_wcs0.25/0.5/1/2 & Number of SPIRE sources within the SPIRE $350 \mu \mathrm{m}$ WCS accuracy $(\times 0.25 / 0.5 / 1 / 2)$. \\
\hline n_SPIRE_SPIRE500_wcs0.25/0.5/1/2 & Number of SPIRE sources within the SPIRE $500 \mu \mathrm{m}$ WCS accuracy $(\times 0.25 / 0.5 / 1 / 2)$. \\
\hline n_PACS_SPIRE250_psf0.25/0.5/1/2 & Number of PACS sources within the SPIRE $250 \mu \mathrm{m}$ PSF $(\times 0.25 / 0.5 / 1 / 2)$ \\
\hline n_PACS_SPIRE350_psf0.25/0.5/1/2 & Number of PACS sources within the SPIRE $350 \mu \mathrm{m}$ PSF $(\times 0.25 / 0.5 / 1 / 2)$. \\
\hline n_PACS_SPIRE500_psf0.25/0.5/1/2 & Number of PACS sources within the SPIRE $500 \mu \mathrm{m}$ PSF $(\times 0.25 / 0.5 / 1 / 2)$ \\
\hline n_PACS_SPIRE250_wcs0.25/0.5/1/2 & Number of PACS sources within the SPIRE $250 \mu \mathrm{m}$ WCS accuracy $(\times 0.25 / 0.5 / 1 / 2)$. \\
\hline n_PACS_SPIRE350_wcs0.25/0.5/1/2 & Number of PACS sources within the SPIRE $350 \mu \mathrm{m}$ WCS accuracy $(\times 0.25 / 0.5 / 1 / 2)$. \\
\hline n_PACS_SPIRE500_wcs0.25/0.5/1/2 & Number of PACS sources within the SPIRE $500 \mu \mathrm{m}$ WCS accuracy $(\times 0.25 / 0.5 / 1 / 2)$. \\
\hline n_MIPS_SPIRE250_psf0.25/0.5/1/2 & Number of MIPS sources within the SPIRE $250 \mu \mathrm{m}$ PSF $(\times 0.25 / 0.5 / 1 / 2)$ \\
\hline n_MIPS_SPIRE350_psf0.25/0.5/1/2 & Number of MIPS sources within the SPIRE $350 \mu \mathrm{m}$ PSF $(\times 0.25 / 0.5 / 1 / 2)$. \\
\hline n_MIPS_SPIRE500_psf0.25/0.5/1/2 & Number of MIPS sources within the SPIRE $500 \mu \mathrm{m}$ PSF $(\times 0.25 / 0.5 / 1 / 2)$ \\
\hline n_MIPS_SPIRE250_wcs0.25/0.5/1/2 & Number of MIPS sources within the SPIRE $250 \mu \mathrm{m}$ WCS accuracy $(\times 0.25 / 0.5 / 1 / 2)$. \\
\hline n_MIPS_SPIRE350_wcs0.25/0.5/1/2 & Number of MIPS sources within the SPIRE $350 \mu \mathrm{m}$ WCS accuracy $(\times 0.25 / 0.5 / 1 / 2)$. \\
\hline n_MIPS_SPIRE500_wcs0.25/0.5/1/2 & Number of MIPS sources within the SPIRE $500 \mu \mathrm{m}$ WCS accuracy $(\times 0.25 / 0.5 / 1 / 2)$. \\
\hline n_IRAC_SPIRE250_psf0.25/0.5/1/2 & Number of IRAC sources within the SPIRE $250 \mu \mathrm{m}$ PSF $(\times 0.25 / 0.5 / 1 / 2)$ \\
\hline n_IRAC_SPIRE350_psf0.25/0.5/1/2 & Number of IRAC sources within the SPIRE $350 \mu \mathrm{m}$ PSF $(\times 0.25 / 0.5 / 1 / 2)$. \\
\hline n_IRAC_SPIRE500_psf0.25/0.5/1/2 & Number of IRAC sources within the SPIRE $500 \mu \mathrm{m}$ PSF $(\times 0.25 / 0.5 / 1 / 2)$ \\
\hline n_IRAC_SPIRE250_wcs0.25/0.5/1/2 & Number of IRAC sources within the SPIRE $250 \mu \mathrm{m}$ WCS accuracy $(\times 0.25 / 0.5 / 1 / 2)$. \\
\hline n_IRAC_SPIRE350_wcs0.25/0.5/1/2 & Number of IRAC sources within the SPIRE $350 \mu \mathrm{m}$ WCS accuracy $(\times 0.25 / 0.5 / 1 / 2)$. \\
\hline n_IRAC_SPIRE500_wcs0.25/0.5/1/2 & Number of IRAC sources within the SPIRE $500 \mu \mathrm{m}$ WCS accuracy $(\times 0.25 / 0.5 / 1 / 2)$. \\
\hline
\end{tabular}


Table C5. Redshift and properties.

\begin{tabular}{|c|c|}
\hline Entry name & Description \\
\hline object & ID of the galaxy in the parent catalogue. \\
\hline z_phot & EAZY $z_{\text {phot }}$ \\
\hline Z_spec & Spectroscopic redshift. \\
\hline Z_spec_flag & Quality of the $z_{\text {spec }}$. Values $>2$ mean reliable. \\
\hline stellar_mass & Stellar mass in $\log _{10} \mathcal{M}_{*} / M_{\odot}$ \\
\hline L_TIR & Total IR luminosity $(8-1000 \mu \mathrm{m})$ in $L_{\odot}$, from the best-fitting template (Draine \& Li 2007). \\
\hline SFR_UV & Star formation rate $\left(M_{\odot} \mathrm{yr}^{-1}\right)$ from the rest-frame monochromatic luminosity at $2800 \AA$. \\
\hline SFR_UV_corr & $\begin{array}{l}\text { Star formation rate }\left(M_{\odot} \mathrm{yr}^{-1}\right) \text { from the rest-frame monochromatic luminosity at } 2800 \AA \text {. } \\
\text { Corrected by extinction using } \mathcal{A}_{\mathrm{UV}}=(1.76 \pm 0.04)+(0.20 \pm 0.02) \beta\end{array}$ \\
\hline SFR_TIR & Star formation rate $\left(M_{\odot} \mathrm{yr}^{-1}\right)$ from the L_TIR. \\
\hline$U$ & Rest-frame $U$ absolute magnitude from best-fitting template. \\
\hline$V$ & Rest-frame $V$ absolute magnitude from best-fitting template. \\
\hline$J$ & Rest-frame $J$ absolute magnitude from best-fitting template. \\
\hline
\end{tabular}

${ }^{1}$ Dipartimento di Fisica e Astronomia 'G. Galilei', Università degli Studi di Padova, Vicolo dell'Osservatorio 3, I-35122 Padova, Italy

${ }^{2}$ Departamento de Astronomía y Astrofísica, Universidad Complutense de Madrid, Av. Complutense s/n, C.P., E-28040 Madrid, Spain

${ }^{3}$ Centro de Astrobiología, Instituto Nacional de Técnica Aeroespacial, Carretera de Ajalvir km 4, Torrejón de Ardoz, E-28850 Madrid, Spain

${ }^{4}$ ESA/Space Telescope Science Institute (STSCI), 3700 San Martin Drive, Baltimore, MD 21218, USA

${ }^{5}$ Steward Observatory, University of Arizona, 933 N. Cherry Ave, Tucson, AZ 85721, USA

${ }^{6}$ INAF - Osservatorio Astronomico di Capodimonte, via Moiariello 16, I-80131 Napoli, Italy

${ }^{7}$ Dipartimento de Fisica e Scienze della Terra, Università degli Studi di Ferrara, via Saragat 1, I-44122 Ferrara, Italy

${ }^{8}$ Laboratoire AIM-Paris-Saclay, CEA/DSM-CNRS-Université Paris Diderot, IRFU/Service d'Astrophysique, CEA Saclay, Orme des Merisiers, F-91191 Gif-sur-Yvette, France

${ }^{9}$ University Observatory Munich, Scheinerstrasse 1, D-81679 Munich, Germany

${ }^{10}$ IPAC, Mail Code 314-6, Caltech, 1200 E. California Blvd., Pasadena, CA 91125, USA

${ }^{11}$ INAF-Osservatorio Astronomico di Trieste, via G. B. Tiepolo 11, I-34131

Trieste, Italy
${ }^{12}$ Institute for Astronomy, University of Hawaii, Honolulu, HI 96822, USA

${ }^{13}$ Centre for Extragalactic Astronomy, Department of Physics, Durham University, South Road, Durham DH1 3LE, UK

${ }^{14}$ Dark Cosmology Centre, Niels Bohr Institute, University of Copenhagen, Juliane Maries Vej 30, DK-2100 Copenhagen, Denmark

${ }^{15}$ Dipartimento di Fisica, Universita degli Studi di Milano, via Celoria 16, I-20133 Milano, Italy

${ }^{16}$ INAF-Osservatorio Astronomico di Brera, via Brera 28, I-20121 Milano, Italy

${ }^{17}$ Department of Physics and Astronomy, PAB, 430 Portola Plaza, Box 951547, Los Angeles, CA 90095-1547, USA

${ }^{18}$ Department of Physics, University of California Davis, 1 Shields Avenue, Davis, CA 95616, USA

${ }^{19}$ Herschel Science Centre, European Space Astronomy Centre, ESA, E28691 Villanueva de la Cañada, Spain

${ }^{20}$ INAF - Osservatorio Astronomico di Padova, vicolo dell'Osservatorio 5, I-35122 Padova, Italy

${ }^{21}$ Center for Detectors, School of Physics and Astronomy, Rochester Institute of Technology, Rochester NY 14623, USA

This paper has been typeset from a $\mathrm{T}_{\mathrm{E}} \mathrm{X} / \mathrm{L} \mathrm{T} \mathrm{E} \mathrm{X}$ file prepared by the author. 